\title{
Precise Theory of the Absorption and Dispersion of Forced Plane Infinitesimal $W$ aves according to the Navier-Stokes Equations*
}

\author{
C. TRUESDELL
}

Graduate Institute for Applied Mathematics, Indiana University, Bloomington, Indiana

\section{Contents}

1. Absorption and dispersion measures for forced waves. . . . . . . . 644

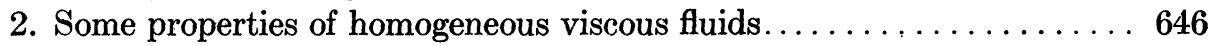

3. Dimensionless parameters. . . . . . . . . . . . . . . . 648

4. History and critique of the theory of absorption and dispersion. . . . 650

5. The present status of ultrasonic absorption and dispersion as a prob-

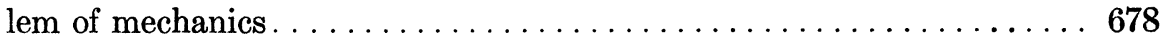

6 . The results in this memoir . . . . . . . . . . . . . . . . 684

7. Derivation of the characteristic equation of Kirchhoff and Langevin... 685

8. Further remarks on the piezotropic case . . . . . . . . . . 687

9. The general case: the two types of waves and the three major types of fluids................................. 690

10. General exact expressions for the absorption and dispersion measures 698

11. Approximation for very low and moderately low frequencies... . . . . 698

12. Approximation for very high and moderately high frequencies...... 702

13. Summary and conjectures. The physical occurrence of the two types of

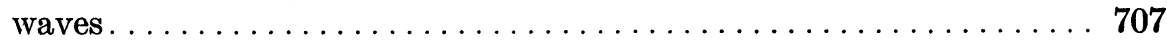

14. A power series expansion and its consequences............ 708

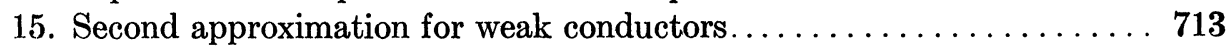

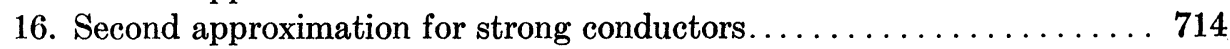

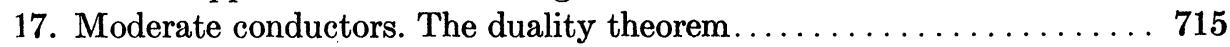

18. First approximation for gases: the pseudopiezic fluids. . . . . . . 718

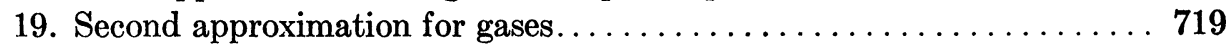

20. The singular fluids . . . . . . . . . . . . . . . . . . . . . 720

21. Tables..................................... 721

* Part of the results below were presented at the Symposium on Ultrasonic Absorption and Dispersion at Brown University, October, 1952, and at the meeting of the American Physical Society at Pennsylvania State College, July, 1953. This memoir was written for the Applied Mathematics Branch, Naval Research Laboratory, Washington, D. C. Some exploratory calculations were made by assistants working under ONR contract at Indiana University. 
1. Absorption and dispersion measures for forced waves. Let a continuous medium be maintained in one-dimensional motion at a certain plane $x=0$ according to the law

$$
\frac{I}{I_{0}}=\Re e^{i \omega t}
$$

where $I$ stands for particle displacement, velocity, acceleration, pressure, density, or temperature, and $I_{0}$ is a corresponding constant amplitude. Whatever be the physical units selected, by considering only excitations in which the various amplitudes $I_{0}$ are sufficiently small, we may render all non-linear terms in the differential equations of motion as small as we please in comparison to the linear ones, and while in the absence of a proper mathematical approximation theorem we cannot actually prove, yet may we with some confidence expect, that the actual motion does not sensibly differ from that which satisfies the differential equations obtained by omitting all non-linear terms from the original system. According to these equations, in which moreover all coefficients are approximated by their values before the medium is excited, the disturbance (1.1) will be propagated as a damped harmonic oscillation:

$$
\frac{I}{I_{0}}=\Re e^{x x+i \omega t}
$$

where $\chi$ is a complex quantity whose value depends on the nature of the medium and on the circular frequency $\omega$ of the excitation.

Let $l_{0}$ be the wave-length corresponding to a certain fixed reference speed $V_{0}$ :

$$
l_{0} \equiv \frac{2 \pi V_{0}}{\omega}
$$

and define the dimensionless quantity

$$
\sigma \equiv l_{0} x=\frac{2 \pi V_{0} x}{\omega} .
$$

The squared ratio $r$ of the actual speed of propagation $V$ to the reference speed $V_{0}$ is a measure of dispersion; it is given by

$$
r \equiv\left(\frac{V}{V_{0}}\right)^{2}=\left(\frac{l_{0} \omega}{V_{0} \Im_{\sigma}}\right)^{2}=\left(\frac{2 \pi}{\Im_{\sigma}}\right)^{2}
$$

The absorption coefficient per reference wave-length $A_{0}$ is given by

$$
A_{0} \equiv l_{0}|\Re \chi|=|\Re \sigma|,
$$

while the true absorption coefficient per wave-length $A$ is given by

$$
A \equiv l|\Re \chi|=\frac{l}{l_{0}}|\Re \sigma|=\frac{l}{l_{0}} A_{0}=\frac{V}{V_{0}} A_{0}=\sqrt{r} A_{0}=2 \pi\left|\frac{\Re \sigma}{\Im_{\sigma}}\right|,
$$


ince the wave-length $l$ corresponding to the actual speed of propagation $V$ is $l=2 \pi V / \omega$. With these definitions, (1.2) becomes

$$
\frac{I}{I_{0}}=e^{ \pm A_{0} \frac{x}{l_{0}}} \cos \omega\left( \pm \frac{x}{V}+t\right)=e^{ \pm A \frac{x}{l}} \cos \omega\left( \pm \frac{x}{V}+t\right)
$$

whence are manifest the reasons for the names assigned to $V, A_{0}$, and $A$.

In experimental work it is usually $V$ and a different absorption coefficient $\alpha$, given by

$$
\alpha \equiv A_{0} / l_{0}=A / l=|\Re \chi|
$$

whose measured values, expressed in $\mathrm{cm}^{-1}$, are reported. Sometimes also $\alpha / \nu^{2}=$ $4 \pi^{2} \alpha / \omega^{2}=4 \pi^{2} A_{0} /\left(l_{0} \omega^{2}\right)$ is used.

For theoretical calculation, however, the ratio $r$ and yet a fourth absorption coefficient $a$, given by

$$
a \equiv \frac{V_{0}}{V} A_{0}=\frac{A_{0}}{\sqrt{r}}=\left(\frac{V_{0}}{V}\right)^{2} A=\frac{A}{r}=\frac{|\Re \sigma \Im \sigma|}{2 \pi},
$$

are more convenient. From these two, all others are easily calculated.

If dispersion be negligible, $A \approx A_{0} \approx a$.

We shall have a good many square roots to calculate. We reserve the notation $\checkmark$ to denote the principal determination, namely, the value assumed by the branch of $z^{\frac{1}{2}}$ which is positive when $z=+1$, the $z$-plane being supposed cut along the negative real axis. Points on the negative real axis itself will be assigned the argument $\pi$; thus $\sqrt{-K^{2}} \equiv i K$ when $K$ is real. The exponent $\frac{1}{2}$ will denote both possible values of the square root: $z^{\frac{3}{2}}= \pm \sqrt{z}$.

With these conventions we have, when $E$ and $F$ are real and $F \neq 0$,

$$
\begin{gathered}
\sqrt{2} \sqrt{E+i F}=\sqrt{\sqrt{E^{2}+F^{2}}+E}+i(\operatorname{sgn} F) \sqrt{\sqrt{E^{2}+F^{2}}-E} \\
\frac{\sqrt{2}}{\sqrt{E+i F}}=\frac{\sqrt{\sqrt{E^{2}+F^{2}}+E}-i(\operatorname{sgn} F) \sqrt{\sqrt{E^{2}+F^{2}}-E}}{\sqrt{E^{2}+F^{2}}}
\end{gathered}
$$

We shall see later that it is always easy to find real $G$ and $H$ such that

$$
-\left(\frac{2 \pi}{\sigma}\right)^{2}=G+i H
$$

Then, whatever the values and signs of $G$ and $H$ and whichever of the two roots $\sigma$ of (1.13) be employed, by (1.12) it follows that

$$
\frac{|\Re \sigma|}{2 \pi}=\frac{\sqrt{\sqrt{G^{2}+H^{2}}-G}}{\sqrt{2} \sqrt{G^{2}+H^{2}}}, \quad \frac{|\Im \sigma|}{2 \pi}=\frac{\sqrt{\sqrt{G^{2}+H^{2}}+G}}{\sqrt{2} \sqrt{G^{2}+H^{2}}} .
$$

(While the case $F=0$ is not included in (1.12), inspection shows that (1.14) 
remains valid when $H=0$.) From (1.14) and the definitions of the various absorption and dispersion measures we have then

$$
a=\frac{\pi H}{G^{2}+H^{2}}, \quad r=\frac{2\left(G^{2}+H^{2}\right)}{\sqrt{G^{2}+H^{2}}+G}, \quad A=r Q, A_{0}^{2}=r Q^{2}=A Q .
$$

These formulae are the basis of all subsequent calculations.

It will appear later that in fluids there are two types of waves, with two different sets of measures. The reference speed $V_{0}$, which so far has been merely an arbitrary constant, will soon be fixed once and for all at the classical value of the speed of sound for the fluid in question. This speed will then turn out to be a meaningful reference speed for one type of waves, but not for the second, for which, indeed, no particular reference value will recommend itself. The quantities $a$ and $A$ are independent of $V_{0}$, and hence will retain their significance equally for both types of fluids. Not so, however, with $r$ and $A_{0}$, which depend upon $V_{0}$. We shall find it convenient to replace these by the relative dispersion

$$
\Omega \equiv \frac{r^{\prime}}{r}=\left(\frac{V^{\prime}}{V}\right)^{2}
$$

and the relative absorption

$$
Q \equiv \frac{\left|\Re \chi^{\prime}\right|^{2}}{|\Re \chi|^{2}}=\left(\frac{A_{0}^{\prime}}{A_{0}}\right)^{2}=\Omega\left(\frac{\alpha^{\prime}}{Q}\right)^{2},
$$

where primes distinguish the measures for the second type of waves from those for the first. The physical significance of these two measures is obvious: when $\Omega$ is large, a wave of the second type rapidly overtakes one of the first, and when $Q$ is large, a wave of the second type is damped much more severely in travelling any given distance than is one of the first type.

2. Some properties of homogeneous viscous fluids. A fluid is homogeneous if there exist a caloric equation of state

$$
\epsilon=\epsilon(\eta, v)
$$

which connects the specific internal energy $\epsilon$ per unit mass, the specific entropy $\eta$, and the specific volume $v$. The density $\rho$ is $v^{-1}$. For such a fluid, pressure $p$, absolute temperature $\theta$, and specific heats $c_{p}$ and $c_{v}$ are defined by

$$
p \equiv-\left(\frac{\partial \epsilon}{\partial v}\right)_{\eta}, \quad \theta \equiv\left(\frac{\partial \epsilon}{\partial \eta}\right)_{v}, \quad c_{p} \equiv \theta\left(\frac{\partial \eta}{\partial \theta}\right)_{p}, \quad c_{v} \equiv \theta\left(\frac{\partial \eta}{\partial \theta}\right)_{v}
$$

the ratio of the last being $\gamma \equiv c_{p} / c_{v}$. From (2.2) we have in general $p=p(v, \eta)$, $\theta=\theta(v, \eta)$, whence by elimination of $\eta$ follows a thermal equation of state: $p=$ $p(v, \theta)$. The coefficient of thermal expansion is given by

$$
\alpha_{\theta} \equiv \frac{1}{v}\left(\frac{\partial v}{\partial \theta}\right)_{p}
$$


A fluid is piezotropic if (2.1) be of the functional form

$$
\epsilon=H(\eta)+\Upsilon(v)
$$

for such a fluid (2.2) yields

$$
p=-\Upsilon^{\prime}(v), \quad \theta=\mathrm{H}^{\prime}(\eta)
$$

Many liquids can be represented in first approximation as piezotropic. A fluid in which $p \equiv 0$, i.e. $\Upsilon=$ const., is incompressible; what is called pressure in an incompressible fluid is not given by $(2.2)_{1}$ and is not a thermodynamic variable at all. We are not concerned with incompressible fluids in this memoir. It is easy to see that from either of (2.5) by aid of (2.2) follows (2.4). It is easy to see also that $a$ fluid is piezotropic if and only if $\gamma=1$, or, equivalently, $\alpha_{\theta}=0$.

A fluid which is not piezotropic is tri-variate; for such a fluid, none of the equations of state degenerate into relations connecting only two variables.

A perfect gas is a fluid whose thermal equation of state is $p=R \rho \theta, R$ being a constant. Hence it is easy to show that $c_{v}=c_{v}(\theta), c_{p}-c_{v}=R$; if $c_{v}=$ const., then $\epsilon=c_{v} \theta$.

The stress tensor $t_{j}^{i}$ and heat flux vector $q_{i}$ in an isotropic viscous fluid are given in first approximation by the Newton-Cauchy-Poisson law and by Fourier's law, respectively:

$$
t_{j}^{i}=-p \delta_{j}^{i}+\lambda d_{k}^{k} \delta_{j}^{i}+2 \mu d_{j}^{i}, \quad q_{i}=-\kappa \theta_{, i},
$$

where $d_{j}^{i}$ is the rate of deformation tensor, $\lambda$ and $\mu$ are the two viscosities, and $\kappa$ is the thermal conductivity. The dynamical equations resulting from $(2.6)_{1}$ are called the Navier-Stokes equations.

If the effect of viscosity and heat conduction be neglected, so that $t_{j}^{i}=-p \delta_{j}^{i}$, $q_{i}=0$, then the speed of sound $V_{0}$ in a homogeneous fluid is given by

$$
V_{0}^{2}=\left(\frac{\partial p}{\partial \rho}\right)_{\eta}
$$

Since the present memoir concerns only fluids, the reference speed $V_{0}$ in the formulae of $\$ 1$ will always be supposed given by (2.7).

In a perfect gas with constant specific heats we have $p \propto \rho^{\gamma}$ when $\eta=$ const., whence (2.7) yields the Laplace-Poisson formula

$$
V_{0}^{2}=\frac{\gamma p}{\rho} \text {. }
$$

In liquids such a formula does not hold at all. For example, many liquids can be approximated as polytropic, i.e. as piezotropic fluids with equations of state $(2.5)_{1}$ of the very special type $p \propto\left(\rho-\rho_{0}\right)^{\delta}$. For such a fluid we have by (2.7) $V_{0}^{2}=p \delta /\left(\rho-\rho_{0}\right)$, but $\delta$ is not to be confused with $\gamma$, since in a piezotropic fluid $\gamma=1$. For liquids $\gamma$ is easiest determined from the thermodynamic identity

$$
\gamma-1=\frac{\theta \alpha_{\theta}^{2} V_{0}^{2}}{c_{p}}
$$


In water, for example, we have $\delta \approx 7$ but $\gamma \approx 1.0006$, indicating that very little error would be made in regarding water as a piezotropic fluid.

The equation (2.9) makes plain that $\gamma \geqq 1$ in all fluids. The piezotropic fluid is thus a limiting case. On the basis of kinetic theory arguments it is commonly believed that $1<\gamma \leqq 1 \frac{2}{3}$ for gases. Although no measured values of $\gamma$ much above 2 have been reported for any fluid, there seems to be no thermodynamic reason for assigning a finite upper bound, and in this memoir the range of variation of $\gamma$ is taken as $1 \leqq \gamma<\infty$.

3. Dimensionless parameters. From the moduli $\lambda, \mu, \kappa, c_{p}, c_{v}$ which describe the gross response of a fluid to change of shape or temperature it is possible to form three independent dimensionless ratios. In addition to $\gamma$, these may be taken as the Prandtl number $P$ and the viscosity number $v$, defined by

$$
\frac{1}{P} \equiv \frac{\kappa}{\mu c_{p}}, \quad v \equiv 2+\frac{\lambda}{\mu} .
$$

From the second law of thermodynamics follows $0 \leqq P^{-1} \leqq \infty, \frac{4}{3} \leqq v \leqq \infty$.

The value $V=\frac{4}{3}$ is equivalent to the Stokes relation $3 \lambda+2 \mu=0$, which is implied by the definitions used in the kinetic theory of monatomic gases. ${ }^{1}$

To describe the resistance of the fluid to oscillations of given frequency, it is necessary to introduce a dimensionless parameter depending upon $\omega$. We shall see that the absorption and dispersion of plane waves in pure fluids is most conveniently studied in terms of the three parameters $\gamma, Y, X$, where the thermoviscous number $Y$ and the frequency number $X$ are defined by

$$
Y \equiv \frac{1}{P \nu}=\frac{\kappa}{(\lambda+2 \mu) c_{p}}, \quad X \equiv v \frac{\mu \omega}{\rho V_{0}^{2}}=\frac{(\lambda+2 \mu) \omega}{\rho V_{0}^{2}} .
$$

All absorption and dispersion measures will be shown to be functions of $\gamma, Y$, and $X$ only. Since $\gamma$ and $Y$ are material constants, independent of the excitation, we shall find it convenient to speak of "the fluid $\langle\gamma, Y\rangle$ " and to represent each fluid as a single point in a $\gamma-Y$ quarter-plane.

The practical difficulty in using $X$ and $Y$ is that both depend upon the second viscosity, $\lambda$, which has been the subject of a long controversy. For some purposes we shall therefore use a counterpart defined in terms of shear viscosity only:

$$
S \equiv \frac{X}{\mathcal{V}}=\frac{\mu \omega}{\rho V_{0}^{2}}
$$

It is appropriate to call $S$ the Stokes number.

Table 3.1 shows numerical values for the various dimensionless parameters in certain fluids. The values assigned to $\lambda$ in order to obtain $v$ are merely hypo-

\footnotetext{
${ }^{1} C f$. "On the viscosity of fluids according to the kinetic theory," Z. Phys. 131, 273-289 (1.952).
} 
thetical, ${ }^{2}$ and the purpose of the table is only to show orders of magnitude. Unfortunately, adequate data on highly viscous fluids and on molten metals is not available. For the former, $S(1)$ will be far larger than for any existing entry, and $\nu(1)$ far smaller. For the latter, $P^{-1}$ and $\gamma$ may be very large.

TABLE 3.1

\begin{tabular}{|c|c|c|c|c|c|c|c|c|}
\hline Fluid & Conditions & $\gamma$ & $\mathbf{P}^{-1}$ & U & $Y$ & $S(1)$ & $X(1)$ & $v(1)$ \\
\hline Acetic acid. & $20^{\circ} \mathrm{C}$ & 1.23 & .075 & 35 & .002 & $3.3 \cdot 10^{-6}$ & $1.2 \cdot 10^{-4}$ & 8700 \\
\hline Acetone.......... & $20^{\circ} \mathrm{C}$ & 1.39 & .252 & 5 & .05 & $1.9 \cdot 10^{-5}$ & $9.5 \cdot 10^{-5}$ & 104 . \\
\hline Benzene........ & $10^{\circ} \mathrm{C}$ & 1.44 & .110 & 110 & .001 & $2.9 \cdot 10^{-6}$ & $3.2 \cdot 10^{-4}$ & 3100 \\
\hline Carbon disulfide... & $15^{\circ} \mathrm{C}$ & 1.56 & .400 & 200 & .002 & $1.4 \cdot 10^{-6}$ & $2.8 \cdot 10^{-4}$ & 3600 \\
\hline Carbon tetrachlo- & $20^{\circ} \mathrm{C}$ & 145 & 138 & 30 & 005 & $4 \cdot 10^{-5}$ & $13.10^{-4}$ & 7700 \\
\hline Ethyl acetate. . . & $20^{\circ} \mathrm{C}$ & 1.50 & .018 & 18 & .001 & $1.7 \cdot 10^{-6}$ & $3.1 \cdot 10^{-5}$ & $3.3 \cdot 10^{4}$ \\
\hline Ethyl alcohol.. & $20^{\circ} \mathrm{C}$ & 1.19 & .063 & 5.8 & .01 & $7.0 \cdot 10^{-6}$ & $4.1 \cdot 10^{-5}$ & $3.6 \cdot 10^{4}$ \\
\hline Ethyl formate. & $24^{\circ} \mathrm{C}$ & 1.81 & .200 & 28 & .007 & $8.7 \cdot 10^{-6}$ & $2.4 \cdot 10^{-4}$ & 4200 \\
\hline Glycerin. . . . & $20^{\circ} \mathrm{C}$ & 1.11 & $10^{-4}$ & 1.7 & $6 \cdot 10^{-5}$ & .0014 & .0024 & 420 \\
\hline Mercury.. & $15^{\circ} \mathrm{C}$ & 1.15 & 38.3 & 2.5 & 15 & $4.7 \cdot 10^{-7}$ & $1.2 \cdot 10^{-6}$ & $8.5 \cdot 10^{5}$ \\
\hline Olive oil. & $20^{\circ} \mathrm{C}$ & 1.13 & .10 & 1.6 & .006 & $2.9 \cdot 10^{-4}$ & $4.6 \cdot 10^{-4}$ & $2.2 \cdot 10^{3}$ \\
\hline Toluene. . & $20^{\circ} \mathrm{C}$ & 1.36 & .142 & 14 & .01 & $2.4 \cdot 10^{-6}$ & $3.4 \cdot 10^{-8}$ & $3.1 \cdot 10^{4}$ \\
\hline Water..... & $15^{\circ} \mathrm{C}$ & 1.0006 & .121 & 4.4 & .03 & $3.4 \cdot 10^{-6}$ & $1.5 \cdot 10^{-5}$ & $6.7 \cdot 10^{4}$ \\
\hline Liquid argon. . . & $85^{\circ} \mathrm{K}$ & 2.22 & .40 & $4 / 3$ & .3 & $1.6 \cdot 10^{-6}$ & $2.1 \cdot 10^{-6}$ & $4.7 \cdot 10^{5}$ \\
\hline Liquid nitrogen. . . & $74^{\circ} \mathrm{K}$ & 1.99 & .57 & 1.7 & .3 & $1.5 \cdot 10^{-6}$ & $2.5 \cdot 10^{-6}$ & $4.1 \cdot 10^{5}$ \\
\hline Liquid oxygen.... & $78^{\circ} \mathrm{K}$ & 1.74 & .44 & 1.7 & .3 & $1.4 \cdot 10^{-6}$ & $2.4 \cdot 10^{-6}$ & $4.2 \cdot 10^{5}$ \\
\hline Air. . . . . . . & NTP & 1.40 & 1.38 & 1.9 & .7 & $7.7 \cdot 10^{-4}$ & .0015 & 670 \\
\hline Argon. . . . . . . . & NTP & 1.67 & 1.42 & $4 / 3$ & 1.1 & $7.9 \cdot 10^{-4}$ & .0011 & 950 \\
\hline Carbon dioxide.... & NTP & 1.30 & 1.26 & 1000 & .001 & $6.6 \cdot 10^{-4}$ & .66 & 1.5 \\
\hline Helium . . . . . . . . . & NTP & 1.66 & 1.43 & $4 / 3$ & 1.1 & $1.4 \cdot 10^{-5}$ & $1.8 \cdot 10^{-5}$ & $5.6 \cdot 10^{4}$ \\
\hline Hydrogen. . . & NTP & 1.41 & 1.67 & 33 & .05 & $3.8 \cdot 10^{-5}$ & .0012 & 870 \\
\hline Neon...... & NTP & 1.70 & 1.49 & 2 & .7 & .0011 & .0022 & 450 \\
\hline Nitrogen. . . & NTP & 1.41 & 1.37 & 1.8 & .8 & $7.4 \cdot 10^{-4}$ & .0012 & 870 \\
\hline Nitric oxide. & NTP & 1.30 & 1.25 & 50 & .006 & $6.6 \cdot 10^{-4}$ & .033 & 30 \\
\hline Oxygen....... & NTP & 1.40 & 1.38 & 1.7 & .8 & $8.6 \cdot 10^{-4}$ & .0015 & 690 \\
\hline Sulfur dioxide..... & NTP & 1.29 & 1.11 & 100 & .001 & $5.6 \cdot 10^{-4}$ & .056 & 18 \\
\hline
\end{tabular}

Constants of certain fluids

$\mathcal{V} \equiv 2+\lambda / \mu$, determined from Liebermann's streaming measurements when possible (see $\$ 4$ ), otherwise from excess absorption data by means of (4.32).

$S(1)=$ value of $S$ at a frequency of 1 megacycle.

$X(1)=$ value of $X$ at a frequency of 1 megacycle, using the above-mentioned value of $\mathcal{U}$.

$\nu(1)=$ frequency in megacycles at which $X=1$.

By the terms "low frequency," "moderate frequency," "high frequency," we shall mean frequencies such that for the fluid in question $X \ll 1, X \approx 1$, or $X \gg 1$.

As appears from Table 3.1, most of the ultrasonic experiments performed up

2 The methods used are explained at the foot of the table. As we shall see in $\$ 5$, both these methods are questionable. 
to the present concern only very low frequencies in the sense of the present work. Notice, however, that in the case of a perfect gas we may put (2.9) into $(3.2)_{2}$ to obtain

$$
X_{\mathrm{gas}}=\frac{(\lambda+2 \mu) \omega}{\gamma p}=\nu \frac{\mu \omega}{\gamma p}
$$

Thus a k-fold decrease in pressure is equivalent to a $k$-fold increase in frequency. By sufficient rarefaction, then, arbitrarily high values of the frequency number $X$ may be obtained. The parameter $X$ (or $S$ ) is thus a dimensionless quantity proportional to the ratio $\nu / p$, in terms of which experimental results are now often reported. Moreover, from (3.2) 3 it is plain that for any fluid, the greater is the second viscosity $\lambda$ in comparison to $\mu$, the greater is the frequency number $X$ which corresponds to a given angular frequency $\omega$.

4. History and critique of the theory of absorption and dispersion. The history of the theory of absorption and dispersion of sound has been written many times, but never completely or even correctly. The following outline is limited to the theory of plane infinitesimal waves. Whenever possible, we use the notations of the previous sections.

A. Hydrodynamical period: the four basic papers.

The problem was first attacked by Stokes in his great memoir on viscosity and elasticity. ${ }^{1} \mathrm{He}$ derived a differential equation governing the propagation of infinitesimal plane waves in a piezotropic fluid and found the solution representing the decay of a free wave whose wave length is $l$. If we write

$$
\frac{I}{I_{0}}=e^{-\overline{\mathbf{A}}^{2 \pi V_{0}} t} \cos \frac{2 \pi}{l}( \pm x+V t), \quad \bar{S} \equiv \pi v \frac{\mu}{\rho l V_{0}}=\pi \frac{\lambda+2 \mu}{\rho l V_{0}},
$$

then Stokes's result is the special case $v=\frac{4}{3}$ of the formulae

$$
\bar{A}=\bar{S}, \quad r=1-\bar{S}^{2}=1-\bar{A}^{2},
$$

which are exact consequences of the linearized equations of motion. From the observed fact that the speed of an audible sound is sensibly the same, whatever be its pitch, Stokes concluded that in air $\bar{A} \ll 1$ for these frequencies. Note that this analysis refers entirely to free waves, not to forced ones. ${ }^{2}$ The results concerning forced waves which are cited in the current ultrasonic literature as from this paper are not contained in it at all. Neither would it be correct

1 "On the theories of the internal friction of fluids in motion, and of the equilibrium and motion of elastic solids," Trans. Cambr. Phil. Soc. 8 (1844-1849), 287-319 (1845)= Papers 1, 171-189. See \$7.

2 In his later paper, "An examination of the possible effect of radiation of heat on the propagation of sound," Phil. Mag. (4) 1, 305-317 (1851) = (with some omissions) Papers 3, 142-154, STokes pointed out the difference between the two kinds of measure, but so far as I know he never published any analysis pertaining to forced waves in a viscous fluid. 
to say that from (4.2) the measures for forced waves may be derived, since in general the two sets of measures are altogether different. ${ }^{3}$

From (4.2) it follows that viscosity induces normal dispersion of free waves, the effect increasing sharply as the waves grow shorter. For waves short enough that $\bar{S} \geqq 1$ the motion is overdamped and the disturbance does not propagate at all; in this case (4.2) is to be replaced by

$$
\bar{A}=\bar{S}+\sqrt{\bar{S}^{2}-1}, \quad V=0 .
$$

The critical wave length $l_{c}$ is given by

$$
l_{c}=\pi v \frac{\mu}{\rho V_{0}},
$$

corresponding to $\bar{S}=\bar{A}=1$. Numerical estimates ${ }^{4}$ indicate critical lengths of the order of $10^{-5} \mathrm{~cm}$ for strongly absorbing liquids, but possibly as little as $10^{-2} \mathrm{~cm}$ in polyatomic gases.

The theory of forced waves was also indeed initiated by Stokes in a later paper, ${ }^{5}$ but there he considered only the effect of radiation, not of viscosity. Adopting Newton's law of cooling, he supposed that the relatively small excess $\theta_{0} \Theta$ of the temperature above that in the unexcited fluid gave rise to an effective heat source distribution:

$$
q_{. i}^{i}=\rho c_{v} q \theta_{0} \theta,
$$

where $q$ is a non-negative coefficient. While he assumed $p v=f(\theta)$, his result can be shown to hold nevertheless for fluids with arbitrary equation of state. If we write $S^{\prime} \equiv \omega / q$, then it is equivalent to

$$
\begin{aligned}
& r=\frac{2}{\gamma} \frac{1+\gamma^{2}{S^{\prime}}^{2}}{1+\gamma{S^{\prime 2}}^{2}+\sqrt{1+\gamma^{2}{S^{\prime 2}}^{2}} \sqrt{1+{S^{\prime 2}}^{2}}}, \\
& a=\frac{\pi \gamma(\gamma-1) S^{\prime}}{1+\gamma^{2} S^{2}}, \\
& A_{0}^{2}=2 \pi^{2} \gamma \frac{\sqrt{1+S^{\prime 2}} \sqrt{1+\gamma^{2} S^{\prime 2}}-1-\gamma{S^{\prime}}^{2}}{1+\gamma^{2} S^{\prime 2}}, \\
& A=\frac{2 \pi(\gamma-1) S^{\prime}}{1+\gamma{S^{\prime}}^{2}+\sqrt{1+S^{\prime 2}} \sqrt{1+\gamma^{2}{S^{\prime 2}}^{\prime 2}}} .
\end{aligned}
$$

\footnotetext{
${ }^{3}$ This fact has recently been noted by R. B. LinDsaY, "Spatial and temporal attenuation of sound," J. Acoust. Soc. 23, 628 (1951). See also $\$ 10$ of Markham, Beyer, \& Lindsay, op. cit. Note 27 below.

${ }^{4}$ See my note, "Longueur critique pour la propagation des ondes libres dans un fluide visqueux," C. R. Acad. Sci. Paris 235, 702-704 (1952).

5 op. cit. Note 2.
} 
These formulae are exact, complete, and final, but apparently latent therein are endless possibilities for misinterpretation, since it is difficult to find in the subsequent literature, even in authoritative review articles, a single correct statement about Stokes's work on radiation.

The dispersion $r$ is a monotone increasing function of $S^{\prime}$, with a single point of inflection and with terminal values $\gamma^{-1}$ and 1 ; thus $V^{2}$ increases steadily from the Newtonian value $V_{0}^{2} / \gamma$ to the Laplace-Poisson value $V_{0}^{2}$. All three absorption coefficients $Q, A_{0}$ and $A$ rise from 0 to single maxima and fall off thereafter to 0 . Though it was not noted by Stokes, we have here the first example of what in recent decades has come to be called a "relaxation effect," but is better designated by the old term resonance (or perhaps, since it indicates an absorption maximum, anti-resonance might be preferable). The respective values of $S^{\prime}$ are

$$
S^{\prime *}=\frac{1}{\gamma}<S^{\prime \#}=\frac{1}{\gamma} \sqrt{\frac{3 \gamma+1}{\gamma+3}}<S^{\prime \prime}=\frac{1}{\sqrt{\gamma}},
$$

with respective maxima

$$
a^{*}=\frac{1}{2} \pi(\gamma-1)<A_{0}^{\#}=\frac{1}{2} \pi(\gamma-1) \sqrt{\frac{2}{\gamma+1}}<A^{\natural}=2 \pi \frac{\sqrt{\gamma}-1}{\sqrt{\gamma}+1} .
$$

It has become fashionable to put relations like (4.7) in terms of "relaxation times," viz.

$$
\tau^{\prime *}=\frac{2 \pi \gamma}{q}>\tau^{\prime \#}=\frac{2 \pi \gamma}{q} \sqrt{\frac{\gamma+3}{3 \gamma+1}}>\tau^{\prime \prime}=\frac{2 \pi}{q} \sqrt{\gamma}
$$

these times being the periods of the waves for which the respective maxima are attained. From (4.8) we see that $\propto, A_{0}$, and $A$ are rather small for the entire range $0 \leqq S^{\prime}<\infty$ unless $\gamma$ is large. For all gases and ordinary liquids $\left(1<\gamma \leqq \frac{5}{3}\right)$ we have $a \leqq \frac{1}{3} \pi, A_{0} \leqq \frac{1}{2} \pi / \sqrt{3}, A \leqq 2 \pi(\sqrt{5}-\sqrt{3}) /(\sqrt{5}+\sqrt{3})$; for most liquids all three coefficients are much smaller.

Now Stokes derived his results on radiation in the course of one of the last controversies regarding the correctness of the Laplace-Poisson formula $V_{0}^{2}=$ $(\partial p / \partial \rho)_{\eta}$. Since audible sounds do not seem to be very greatly damped, Stokes took it as an experimental fact that $A_{0} \approx 0$. From his form of $(4.6)_{3}$, which was not put in dimensionless terms, he concluded that consequently $S^{\prime} \ll 1$ or $S^{\prime} \gg 1$; apparently he did not realize, although it is evident from dimensional considerations, that upper bounds for all three absorption coefficients can be obtained without any knowledge of the value of $q$. Since those bounds vanish with $\gamma-1$, for fluids in which $\gamma-1$ is small his reasoning is therefore faulty; though for fluids with an appreciable value of $\gamma-1$ it would remain correct, setting aside our ignorance of the effect of radiation in non-linear interaction with viscosity and heat conduction.

\footnotetext{
${ }^{6}$ STokes's determination of the maximum for $A_{0}$ is faulty, because it tacitly assumes that what he calls $\mu$ is independent of $S^{\prime}$, which is only rather roughly the case.
} 
The dispersion curve (4.6) ${ }_{1}$ has slope zero at $S^{\prime}=0$ and at $S^{\prime}=\infty$ but at no other value of $S^{\prime}$. Stokes concluded that the only possible way to reconcile this prediction from the theory with the experimental fact that the speeds of audible sounds are independent of their pitch is, again, to suppose either $S^{\prime} \ll 1$ or $S^{\prime} \gg 1$. From the direct consequences of Newton's law of cooling he concluded $S^{\prime} \ll 1$ to be impossible. Then $S^{\prime} \gg 1$ for audible frequencies remained the only alternative.

In such wise, then, did Stokes conclude that the Laplace-Poisson formula must be correct.

The absorption and dispersion of forced waves in a piezotropic viscous fluid were first treated by Stefan. ${ }^{7} \mathrm{He}$ obtained the following exact formulae:

$$
\begin{aligned}
r_{p} & =\frac{2\left(1+X^{2}\right)}{1+\sqrt{1+X^{2}}}, & Q_{p} & =\frac{\pi X}{1+X^{2}}, \\
A_{0 p}^{2} & =\frac{2 \pi^{2}\left(\sqrt{1+X^{2}}-1\right)}{1+X^{2}}, & A_{p} & =\frac{2 \pi X}{1+\sqrt{1+X^{2}}},
\end{aligned}
$$

where we have added subscript $p$ 's because these quantities will be used frequently in the sequel. Stating explicitly that $X \ll 1$, Stefan immediately replaced (4.10) by the approximations ${ }^{8}$

$$
r=1+\frac{3}{4} X^{2}, \quad a=A_{0}=A=\pi X .
$$

For numerical examples he set $\lambda=0$, or, equivalently, $v=2$. He concluded that viscosity produces noticeable absorption, increasing with the pitch of the sound, but negligible dispersion. His paper ends with a derivation of Stokes's earlier results on free waves.

Stefan's work has seldom been quoted, and many authors have derived his results anew. We notice first that both $Q_{p}$ and $A_{0 p}$ increase from 0 to single maxima,

$$
Q_{p}^{*}=\frac{1}{2} \pi<A_{0 p} \#=\frac{1}{2} \pi \sqrt{2},
$$

experienced at

$$
X^{*}=1<X \#=\sqrt{3},
$$

so that the corresponding periods at resonance ("relaxation times") are

$$
\tau_{p}^{*}=\frac{2 \pi(\lambda+2 \mu)}{\rho V_{0}^{2}}>\tau_{p}^{\#}=\frac{2 \pi(\lambda+2 \mu)}{\sqrt{3} \rho V_{0}^{2}} .
$$

7 "Über den Einfluß der inneren Reibung in der Luft auf die Schallbewegung," Sitzber. Akad. Wiss. Wien 532, 529-537 (1866). Our equations (4.10) are his equations (14), put into our notations.

8 In STEFAN's formula equivalent to (4.11), in the middle of his p. 535, the factor $\frac{3}{4}$ has slipped out, doubtless by typographical error. 
The larger is $\lambda$ or $\mu$, the greater the periods at resonance, and the lower the frequency at which resonance occurs; but the height of the resonant peak is independent of the values of $\lambda$ and $\mu$. Accurate experimental location of the values of $S$ at resonance yields a method for experimental determination of $\lambda$ when $\mu$ is known:

$$
\frac{\lambda}{\mu}=-2+\frac{1}{S^{*}}=-2+\frac{\sqrt{3}}{S \#}
$$

As $X \rightarrow \infty$, both $Q_{p}$ and $A_{0 p}$ fall off to zero.

Second, $r_{p}$ and $A_{p}$ are monotone increasing functions of $X$. As $X \rightarrow \infty, r_{p} \rightarrow \infty$ and $A_{p} \rightarrow 2 \pi$. The dispersion induced by viscosity in a piezotropic fluid is thus anomalous at all frequencies, and increases without limit.

The formulae (4.10) are presented graphically in Fig. 4.1.

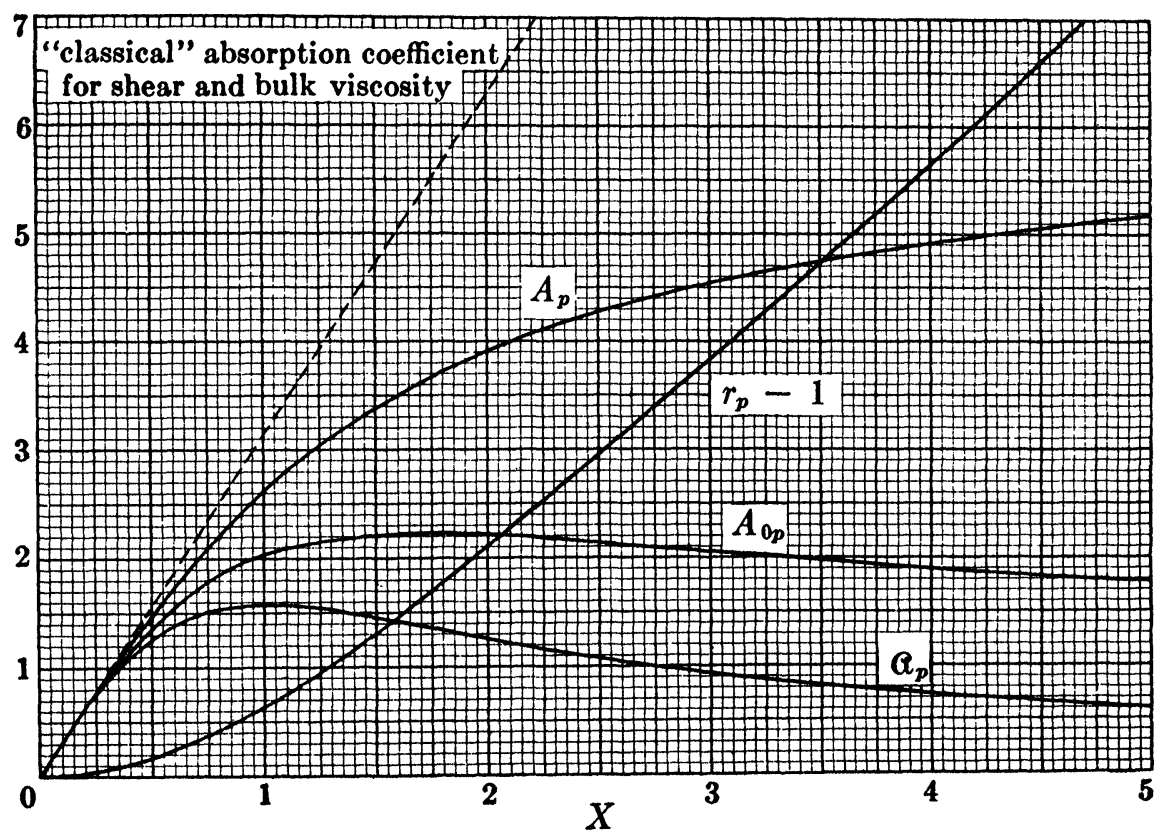

Fig. 4.1

Exact absorption and dispersion measures for pressure waves in a piezotropic fluid (Eq. (4.10))

The definitive treatment of absorption and dispersion in pure fluids was presented by Kirchhoff, ${ }^{9}$ who took both heat conduction and viscosity into account.

9 "Ǔber den Einfluß der Wärmeleitung in einem Gase auf die Schallbewegung," Ann. der Phys. 134, 177-193 (1868) = Abh. 1, 540-556. 
He derived the characteristic equation

$$
(i-\gamma X) X Y\left(\frac{\sigma}{2 \pi}\right)^{4}+(1+i X[1+\gamma Y])\left(\frac{\sigma}{2 \pi}\right)^{2}+1=0 .
$$

While Kirchhoff's treatment is restricted to the case of a perfect gas, many years later Langevin ${ }^{10}$ showed that (4.16) remains valid for pure fluids obeying an arbitrary equation of state. This extension is by no means trivial ( $c f$. $\$ 7$ below) and (4.16) should be called the Kirchhoff-Langevin equation. It will be convenient to refer to quantities associated with the theory of this equation as visco-thermal.

From (4.16) we conclude at once the basic

Characteristic parameter theorem. All visco-thermal absorption and dispersion measures are expressible in terms of the three parameters $X, Y, \gamma$.

The fundamental biquadratic equation (4.16) has two pairs of roots $\pm \sigma$, never coincident and never zero. To each one of these corresponds a solution ${ }^{11}$

$$
\begin{aligned}
& \frac{v}{v_{0}}-1=C e^{\left(\sigma / l_{0}\right) x+i \omega t}, \quad \frac{\theta}{\theta_{0}}-1=-\frac{(\gamma-1) \mathfrak{F}}{1+i \gamma X Y \frac{\sigma^{2}}{4 \pi^{2}}}\left(\frac{v}{v_{0}}-1\right), \\
& \frac{u}{V_{0}}=\frac{2 \pi i}{\sigma}\left(\frac{v}{v_{0}}-1\right), \quad \frac{p}{p_{0}}-1=-\$\left(1+\frac{\gamma-1}{1+i \gamma X Y \frac{\sigma^{2}}{4 \pi^{2}}}\right)\left(\frac{v}{v_{0}}-1\right),
\end{aligned}
$$

where $C$ is an arbitrary complex constant and where $\mathfrak{F}$ and $(\mathbb{f}$ are dimensionless moduli of the fluid, defined by (7.4) and (7.7) 1 below. The general solution is obtained by superposing four particular solutions of the type just written down, one for each value of $\sigma$. Two may be discarded by restricting attention to the half space $x \geqq 0$. Choosing $\sigma$ and $\sigma^{\prime}$ with non-negative real parts, we have then for forced waves

$$
\begin{aligned}
& \frac{v}{v_{0}}-1=C e^{-\left(\sigma / l_{0}\right) x+i \omega t}+B e^{-\left(\sigma^{\prime} / l_{0}\right) x+i \omega t}, \\
& \frac{\theta}{\theta_{0}}-1=-(\gamma-1) \mathfrak{F}\left[\frac{C e^{-\left(\sigma / l_{0}\right) x+i \omega t}}{1+i \gamma X Y \frac{\sigma^{2}}{4 \pi^{2}}}+\frac{B e^{-\left(\sigma^{\prime} / l_{0}\right) x+i \omega t}}{1+i \gamma X Y \frac{\sigma^{\prime 2}}{4 \pi^{2}}}\right] \text {, }
\end{aligned}
$$

etc. The two complex constants $C$ and $B$ may be fixed by requiring the solution to be real and by prescribing the amplitudes of forced oscillation of $v$ and $\theta$ at the plane $x=0$. From these results, as from (4.17), it is plain that oscillation of one of the quantities $v, \theta, u, p$ necessarily entrains oscillation of all the others:

\footnotetext{
10 LANGevin's lectures are written out by P. Biquard, "Sur l'absorption des ondes ultrasonores par les liquides," Ann. de Phys. (11) 6, 195-304 (1936).

11 The fluid is here assumed tri-variate, so $\gamma \neq 1$ and $\mathfrak{F}$ is finite. See $\$ 7$. For a perfect gas, $\mathfrak{F}=\mathbb{G}=1$. For a piezotropic fluid, $\mathfrak{F}=\infty$.
} 
it is impossible in general to separate the pressure waves from the temperature waves; each root of the characteristic equation is associated with oscillations of all the $d y$ namical variables. Alternatively, the fluid in infinitesimal plane motion in the half-space $x \geqq 0$ is equivalent to a dynamical system with two degrees of freedom, for which a set of normal co-ordinates is

$\frac{v}{v_{0}}-1+\frac{1+i \gamma X Y \frac{\sigma^{2}}{4 \pi^{2}}}{(\gamma-1) \mathfrak{F}}\left(\frac{\theta}{\theta_{0}}-1\right), \quad \frac{v}{v_{0}}-1+\frac{1+i \gamma X Y \frac{\sigma^{\prime^{2}}}{4 \pi^{2}}}{(\gamma-1) \mathfrak{F}}\left(\frac{\theta}{\theta_{0}}-1\right)$.

The coefficients of $\theta / \theta_{0}-1$ can become zero or infinite if and only if $(\gamma-1) \mathfrak{F}$ is zero, infinity, or indeterminate; hence $v / v_{0}-1$ and $\theta / \theta_{0}-1$ furnish a set of normal co-ordinates if and only if the fluid be piezotropic.

Now the two different roots $\sigma, \sigma^{\prime}$ give rise to very different absorption and dispersion measures. Which one shall be compared with experimental data? Kirchhoff met and solved this problem in the following way. First, he assumed $\mu, \lambda$, and $\kappa$ to be "unendlich kleine Größen erster Ordnung." Thence he concluded that one of the roots, say $\sigma$, is finite, the other, $\sigma^{\prime}$, infinitely large. The absorption coefficient corresponding to $\sigma^{\prime}$ is then also infinitely large, so that waves corresponding to $\sigma^{\prime}$ may be altogether neglected at moderate distances from the source of disturbance. In our dimensionless notation, Kirchhoff's statement is: $Q=\infty$ when $X=0$. Another way of looking at the matter would consist in noting that as $\gamma \rightarrow 1$, whatever the value of $X$, the absorption and dispersion measures corresponding to $\sigma$ pass continuously into (4.10), which are appropriate to pressure waves in a piezotropic fluid, while the measures corresponding to $\sigma^{\prime}$ pass into those appropriate to thermal waves in a piezotropic fluid. This second view, which will be explored in $\$ 9$, provides a formal way of naming the two types of wave, and leaves open the question as to which of the two is actually observed for large values of $X$, when $\mu, \lambda$, and $\kappa$ cannot justifiably be regarded as "infinitesimals" and hence Kirchhoff's reasoning is no longer applicable.

For the measures corresponding to $\sigma$, retaining only infinitely small quantities of lowest order, Kirchhoff calculated the approximations

$$
r \approx 1, \quad A_{0} \approx \pi X[1+(\gamma-1) Y] .
$$

He noted that there is in fact dispersion, but it is of the order of magnitude of quantities neglected in deriving (4.20), since it depends on the squares of $\mu, \lambda$, and $\kappa$. His paper concludes with a similar discussion of spherical and cylindrical waves.

In the literature one finds not $(4.20)_{2}$ but its special case,

$$
A_{0} \approx \pi S\left[\frac{4}{3}+\frac{\gamma-1}{P}\right]
$$

quoted as the "Stokes-Kirchhoff formula," although it is not to be found in any work of either of the authors to whom it is attributed. To obtain (4.21) from $(4.20)_{2}$ one must assume the Stokes relation, $\mathcal{V}=\frac{4}{3}$. Stokes himself never put 
great faith in this assumption, ${ }^{12}$ and Kirchhoff did not adopt it at all, contenting himself with the passing remark that it holds "nach der Theorie von Stokes" and also "nach der Theorie von Maxwell."

\section{B. Period of qualitative discussion.}

Rayleigh ${ }^{13}$ reproduced in outline the work of Stokes on radiation, errors included. He supplied some numerical values by pure guess, and agreed in Stokes's conclusions, thence replacing $(4.6)_{3}$ by the leading term in its series expansion around $S^{\prime}=\infty$, viz.

$$
A_{0} \approx \frac{\pi}{S^{\prime}}\left(1-\frac{1}{\gamma}\right), \quad \text { or } \quad \alpha=\frac{A_{0}}{l_{0}} \approx \frac{\pi q}{V_{0}}\left(1-\frac{1}{\gamma}\right),
$$

whence has arisen the widespread misconception that radiative absorption is independent of frequency.

At the same time Rayleigh derived a frequency equation for heat conduction alone. It may be obtained formally by holding $X Y$ constant but letting $X \rightarrow 0$ in the Kirchhoff-Langevin equation (4.16). In the piezotropic case we easily find $Q=A_{0}=A=0, r=1$ for the pair of roots corresponding to pure pressure waves, while for the other pair, corresponding to pure thermal waves,

$$
a_{p}^{\prime}=\frac{\pi}{X Y}, \quad r_{p}^{\prime}=2 X Y, \quad A_{p}^{\prime}=2 \pi, \quad A_{0 p}^{\prime 2}=\frac{2 \pi^{2}}{X Y}
$$

Hence, for thermal waves, as frequency increases so does the speed of propagation, and simultaneously $A_{0}^{\prime}$ decreases. These two effects cancel one another in a striking way in the value of $A_{p}^{\prime}$ : the true absorption coefficient per wave length is constant. The formulae (4.23) are presented graphically in Fig. 4.2.

Rayleigh, on the other hand, tacitly selected that pair of roots which as $\gamma \rightarrow 1$ yield undamped waves travelling at speed $V_{0}$, then wrote down the first terms in the expansions for the resulting measures in powers of $X Y$, expressions which may be obtained by supposing $(\gamma-1) Y \gg 1$ in Kirchhoff's formulae (4.20).

12 For a brief history and discussion of the STOKEs relation, see $\$ 61 \mathrm{~A}$ of my paper "The mechanical foundations of elasticity and fluid dynamics," J. Rat. Mech. Anal. 1, 125-300 (1952), 2, 593-616 (1953). See also S. KARIM \& L. Rosenhead, "The second coefficient of viscosity of liquids and gases," Rev. Mod. Phys. 24, 108-116 (1952), and my paper "On the viscosity of fluids according to the kinetic theory," $Z$. Phys. 131, 273-289 (1952).

When we follow up RAYLergh's statement (The Theory of Sound, 2, Cambridge (1878), \$345) ". . . it has been argued with great force by Prof. STokes, that there is no reason why a motion of dilatation uniform in all directions should give rise to viscous force ... " we find STokes's own comment (Papers 3, 136) ". . . I have always felt that the correctness [of my earlier hypothesis] does not rest on as firm a basis as the correctness of the equation of motion of an incompressible fluid ..."

${ }^{13} \mathrm{Op}$. cit. Note 12, \$247. According to the published abstract of a lecture, "The limits of audition," Nature 56, 285-286 (1897) = Proc. R. Inst. 15, 417-418 (1897) = Papers 4, 297-8, RAYLEIGH in 1897 again dismissed radiation as of insignificant effect, but again without any specific data. But $c f$. Note 21 below. 
Thence he quite erroneously concluded: "In truth the conducting power of gases is so feeble, and in the case of audible sounds at any rate the time during which conduction can take place is so short, that disturbance from this cause is not to be looked for." 14

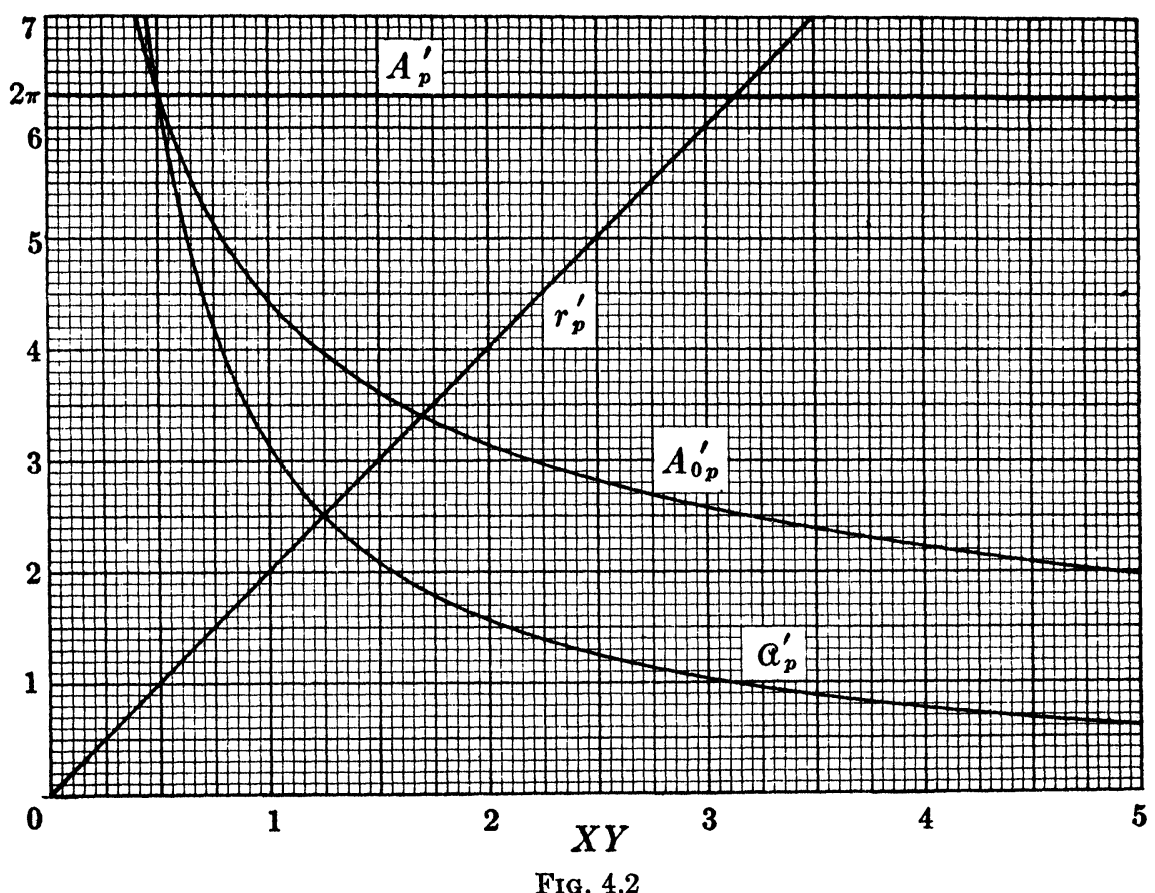

Exact absorption and dispersion measures for thermal waves in a piezotropic fluid (Eq. (4.23))

Rayleigh ${ }^{15}$ also gave equations from which Stefan's results follow, but himself concluded only the approximations $r \approx 1$ and (4.11) 2 . He was the first to point out, though rather vaguely, that rarefying a gas produces the same effect on the absorption and dispersion measures as does increase in frequency.

$\mathrm{Lamb}^{16}$ derived anew the results of Stefan in the special case $V=\frac{4}{3}$, implying that they were due to Stokes. His final formulae are marred by an error in calculation; thus it is fortunate that he did not attempt to interpret them.

14 A similar conclusion was reached by B. BRunhes, "Absorption du son et conductibilité thermique," $J$. de Phys. (3) 6, 289-295 (1897). Although RAYLEIGH in \$\$348-349 of the 1896 edition of his book included a resumé of KIRCHноFF's analysis, from which the opposite conclusion follows, he allowed the passage quoted above to stand unaltered.

15 Op. cit. Note $12, \$ 346$.

${ }^{16}$ A Treatise on the Mathematical Theory of the Motion of Fluids, Cambridge, 1879. See \$183. In later editions this material was omitted. 
The kinetic theory was first applied to the problem by H. A. Lorentz, ${ }^{17}$ who initiated but did not complete a systematic extension of Boltzmann's treatment of polyatomic gases. ${ }^{18} \mathrm{He}$ introduced two different "temperatures," one arising from the translation of the molecules, another from their internal motions. His method was to derive from the kinetic theory hydrodynamical equations, then by means of them to discuss fluid motions. He thus obtained a value for $\gamma$ which differs from $\frac{5}{3}$, according to the amount of the internal temperature, but he assumed $v=\frac{4}{3}$. No more than a special case of the Kirchhoff-Langevin equation can follow from his work.

In his excellent sketch of Kirchhoff's analysis, Lamb ${ }^{19}$ mentioned the effect of putting $\mu=0, \kappa=\infty$ or $\mu=0, \omega \rightarrow \infty$. Equivalently, in (4.16) first hold $X Y$ fixed while letting $X \rightarrow 0$, then let $X Y \rightarrow \infty$. For one pair of roots $Q=A_{0}=$ $A=V=0$; while for the other $Q=A_{0}=A=0, r=\gamma^{-2}$; thus travelling waves are propagated at Newtonian velocity, "since the conditions are now practically isothermal." This result must be regarded with caution, since any non-zero viscosity, however small, negates it: directly from (4.16) it follows that $V \rightarrow \infty$ as $\omega \rightarrow \infty$, for any solution, if $Y$ is finite. However, Lamb's remark suggests the physically evident conclusion that at finite frequencies the effect of heat conduction, which tends to equalize the thermal differences which would arise from strictly isentropic motion, is to decrease the speed of sound. Stefan's result (4.10) shows that the effect of viscosity untrammelled by heat conduction is to increase that speed. Even though for extremely high frequencies $r \rightarrow \infty$ in any case when $\lambda \mu \neq 0$, nevertheless the preceding remarks lead us to expect that at moderate frequencies visco-thermal dispersion will be either anomalous or normal, depending on whether viscosity or heat conduction is the master.

Such was the general confidence in Kirchhoff's result, as misquoted, that for thirty years no experimental test of it was attempted. The first absorption experiment of any kind was performed by A. W. Duff ${ }^{20}$ who compared the measured

17 "Les équations du mouvement des gaz, et la propagation du son suivant la théorie cinétique des gaz," Arch. Néerl. 16, 1-46 (1881) = Works 6, 1-39. German trans. in Abh. Theor. Phys.

18 BoltzmanN's work in this field is summarized in Abschnitt IV of his Vorlesungen über Gastheorie, 2, Leipzig (1898). See esp. \$45.

19 Hydrodynamics, Cambridge, 1895 and later editions. The following misprints are found in the 6th ed. (1932), \$\$359-360:

Eq. (8)

Place:

Eq. (9)

Eq. (14)

l. 4 after Eq. (29)
Instead of:

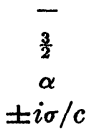

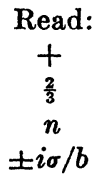

Read:

$\pm i \sigma / b$

LAMB's remark was repeated by ROCARD, loc. cit. Note 47, who drew the conclusion noted in the text above.

20 "The attenuation of sound and the constant of radiation of air," Phys. Rev. 6, 129-139 (1898). 
distance to which the blast of whistles would carry with the absorption formula for spherical waves, carefully rederived for the purpose, but assuming $\vartheta=\frac{4}{3}$. He found that shear viscosity and heat conduction would account for less than half the observed damping, and he attributed the remainder to the effect of radiation.

Rayleigh $^{21}$ designed an experiment on the rise of pressure during recovery of temperature after sudden cooling of a gas by rarefaction, the results of which were satisfactorily explained by heat conduction alone, and from which he concluded the effect of radiation to be negligible. The large absorption measured by Duff he attributed to atmospheric refraction. But he added, "If further experiment should establish a rate of attenuation of the order in question as applicable in uniform air, it will I think be necessary to look for a cause not hitherto taken into account. We might imagine a delay in the equalization of the different sorts of energy in a gas undergoing compression, not wholly insensible in comparison with the time of vibration of the sound. If in the dynamical theory we assimilate the molecules of a gas to hard smooth bodies which are nearly but not absolutely spherical, and trace the effect of a rapid compression, we see that at the first moment the increment of energy is wholly translational and thus produces a maximum effect in opposing the compression. A little later a due proportion of the excess of energy will have passed into rotational forms which do not influence the pressure, and this will accordingly fall off. Any effect of the kind must give rise to dissipation, and the amount of it will increase with the time required for the transformations. ..."

Jeans ${ }^{22}$ claimed that "the propagation of sound is dependent on a transfer of internal energy through collisions, and if this energy is not transferred with sufficient rapidity to keep pace with the transfer of translational energy, complications will arise which are not contemplated by a simple theory of the kind which is given in books on Sound or Hydrodynamics." After considerable calculation, he obtained a result equivalent to augmenting slightly the usual value of the viscosity for monatomic gases.

M. v. Smoluchowski ${ }^{23}$ was the first author to remark that absorption measurements offer a possibility of experimental test of the Stokes relation.

M. Brillouin ${ }^{24}$ in discussing the two viscosities wrote "... nous ne savons actuellement rien sur l'ordre de grandeur relatif des deux termes; rien ne nous autorise encore à effacer le second," and he noted also that only in the combination $\lambda+2 \mu$ do the two viscosities appear in absorption formulae, concluding "Des expériences précises paraissent indispensables."

\footnotetext{
21 "On the cooling of air by radiation and conduction, and on the propagation of sound," Phil. Mag. (5) 47, 308-314 (1899) = Papers 4, 376-381.

${ }_{22}$ The Dynamical Theory of Gases, Cambridge, 1902. See Ch. XVI. In later editions this material was omitted.

23 "Sur les phénomènes aérodynamiques et les effets thermiques qui les accompagnent," Bull. Int. Acad. Sci. Cracovie 1903, 143-182. See \$2.

${ }^{24}$ Leçons sur la viscosité des liquides et des gaz, 2 vols., Paris. 1907. See \$\$25, 30.
} 
P. Lebedew ${ }^{25}$ calculated the numerical value of $A_{0}$ for air from (4.21), using Jeans's value of the viscosity. At his suggestion, absorption measurements for high frequency waves were made by $\mathrm{N}$. Neklepajev. ${ }^{26}$ The value obtained was twice as great as that given by (4.21). Lebedew attributed the discrepancy to the necessary use of the static values for $\kappa, \mu$, and $c_{p}$.

The invention of an ultrasonic generator by $\mathrm{P}$. Langevin gave rise to a host of measurements. Except for a few particular experimental works of definite value for the interpretation of theory, we shall make no attempt to survey this mass of data. ${ }^{27}$ From it, however, emerge three qualitative conclusions: $:^{28}$

1. In nearly all fluids the measured absorption coefficient exceeds the "classical" value given by (4.21). The only exceptions are some of the monatomic gases, perhaps mercury, and perhaps some of the liquefied gases.

2. In nearly all of the relatively few fluids, both liquids and gases, for which data over a considerable frequency range is available, the coefficient $A_{0}$ falls away from linear dependence of $S$ and experiences one or possibly several maxima, ultimately decreasing indefinitely.

3. The dispersion is always anomalous. For some fluids $r$ appears to approach an asymptotic value, usually only slightly greater than 1 . In others, it appears that $r \rightarrow \infty$ as $S \rightarrow \infty$. In most, the data is inconclusive. ${ }^{28 a}$

C. The relaxation theory.

The experimental phenomenon 2 has been named a "relaxation effect." I make no attempt to review in detail what Partington ${ }^{29}$ has called the "exuberant literature" of the subject, resting content to sketch the main ideas.

25 "Die Grenzwerte der kurzesten akustischen Wellen," Ann. der Phys. (4) 35, 171-174 (1911).

26 "Ưber die Absorption kurzer akustischer Wellen in der Luft," Ann. der Phys. (4) 35, 175-181 (1911).

27 Recent surveys are:

L. Bergmann, Der Ultraschall und seine Anwendungen in Wissenschaft und Technik, 5th ed., Zürich, 1949. See Kap. 3b, d.

D. Sette, "L'assorbimento delle onde ultrasonore nei liquidi," Nuovo Cim. (9) 6 Supplemento, (1949).

J. Markham, R. Beyer, \& R. Lindsay, "Absorption of sound in fluids," Rev. Mod. Phys. 23, 353-411 (1951).

S. Petralia, "Velocità e assorbimento di ultrasuoni nei gas," Nuovo Cim. (9) 9 Supplemento, 1-58 (1952).

${ }^{28}$ Only data in which both the independence of absorption and dispersion measures from amplitude and the law of exponential decay with distance is verified is relevant, for only such data can pertain to plane infinitesimal waves. $C f$. Ch. IV, $\$ 1$ and p. 301 of P. BIQUARD, "Sur l'absorption des ondes ultra-sonores par les liquides," Annales de Phys. (11) 6, 195-304 (1936). It should not be forgotten that these conditions are merely necessary, not sufficient.

28a While many writers have confidently drawn dispersion curves which level off at a limit speed, that speed is invariably hypothecated as just beyond existing measurements, and I have never seen a set of experimental points showing independence of $V$ from frequency at high frequencies. I am told, however, that such data exist.

${ }^{29}$ An advanced treatise on physical chemistry 1, London, N. Y., \& Toronto, 1949. See Ch. VII E, §24. 
These are very simple. They were first presented in a path-breaking memoir of Einstein, ${ }^{30}$ where what is now called "irreversible thermodynamics" was first applied to a concrete and specific problem. The analysis proceeds with the clarity and directness characteristic of the author.

Einstein proposed to use the measured wave velocity in a partly dissociated gas in order to determine the reaction rates. ${ }^{31}$ To this end he derived a formula for the speed of sound in a fluid mixture of two reacting components. Supposing the dissipation due to viscosity and heat conduction negligible, he assumed (1) the mixture as a whole obeys the equation of state for a perfect gas; (2) the reaction takes place isentropically; (3) the reaction rates of the components are proportional to their concentrations. Partial temperatures, analogous to those of Lorentz, were introduced in the formulation but eliminated in the end. To put his result into dimensionless form let us write ${ }^{32}$

$$
K^{2} \equiv \frac{1+\frac{A}{B}}{1+\frac{R}{\bar{C}}}, \quad \bar{r} \equiv \frac{V_{0}^{2}}{\frac{p}{\rho}\left(1+\frac{A}{B}\right)}, \quad \bar{X} \equiv \frac{K^{2} \bar{C} \omega}{\kappa_{1} B},
$$

where $A, B, \bar{C}$, and $\kappa_{1}$ are defined in Einstein's paper in terms of the heat of dissociation and the concentrations and heat capacities of the two components. It follows from these definitions that $K^{2}<1$ and $\bar{r}=1$; the latter of these interpretations, however, we shall for the moment refrain from using. Then the square of Einstein's complex velocity equation, separated into real and imaginary parts, may be written

$$
\frac{1}{r}-\frac{r a^{2}}{4 \pi^{2}}=\bar{r} \frac{1+K^{2} \bar{X}^{2}}{1+\bar{X}^{2}}, \quad a=\bar{r}\left(1-K^{2}\right) \frac{\pi \bar{X}}{1+\bar{X}^{2}} .
$$

Einstein himself, setting $\bar{r}=1$ and explicitly neglecting absorption, dropped the second term on the left in $(4.25)_{2}$ and concluded

$$
r \approx \frac{1+\bar{X}^{2}}{1+K^{2} \bar{X}^{2}}, \quad \text { or } \quad r-1 \approx\left(1-K^{2}\right) \frac{\bar{X}^{2}}{1+K^{2} \bar{X}^{2}} .
$$

Herzfeld \& Rice ${ }^{33}$ were apparently the first authors to suggest that Rayleigh and Jeans's notion of different rates of transfer of translational and rotational

\footnotetext{
30 "Schallausbreitung in teilweise dissoziierten Gasen," Sitzber. Akad. Wiss. Berlin Math.-Phys. Kl. 1920, 380-385.

${ }^{31}$ According to Damköhler, op. cit. Note 35 below, this idea had been proposed by NerNst in 1910.

${ }^{32}$ For convenience we give here also the correspondence between the notations in the text and those of KNESER's 1949 Ergebnisheft (see Note 35 below):

$$
\bar{X}=\omega \tau^{\prime}, \quad K^{2}=1-\epsilon,
$$

where $\tau^{\prime}=\tau\left(C_{a}+\Delta\right) /\left(C_{a}+C_{i}+\Delta\right), \epsilon=C_{i} \Delta /\left[\left(C_{a}+C_{i}\right)\left(C_{a}+\Delta\right)\right], \tau$ is the "relaxation time," $C_{a}=$ molal heat for translational degrees of freedom, $C_{i}=$ the same for internal degrees of freedom, $C_{v}=C_{a}+C_{i}, \Delta=C_{p}-C_{i}$.

${ }^{33}$ "Dispersion and absorption of high frequency sound waves," Phys. Rev. (2) 31, 691695 (1928).
} 
energy could be expressed by Lorentz's partial temperatures, then put into mathematical form analogous to Einstein's. Indeed, Einstein's variables as they stand may be given different interpretations to represent various sorts of simple exchange processes in a two component mixture - the components may be aggregates of two types of molecules, atoms excited at two different energy levels, ions in a solution, etc., etc. It is not difficult also to generalize the analysis to the case of an arbitrary equation of state, the exact results again being of the form (4.25), with more elaborate interpretations for the constants. Herzfeld \& Rice were also the first to introduce formally a time $\tau$ "measuring the rate of exchange between external and internal degrees of freedom." This "relaxation time," as it is now called, necessarily figures in the form $\bar{X}=k \tau \omega$ in all results obtainable by the relaxation theory, $\bar{X}$ being the dimensionless frequency occurring in Einstein's equations and $k$ a dimensionless constant.

It is only the concepts of quantum theory which make it reasonable to treat a chemically pure gas, when subjected to a periodic disturbance, as a simple mixture of two or more distinct components, excited in various discrete energy states. Beyond this physical argument, however, most of the authors writing on the relaxation theory have made little or no use of quantum mechanics. It would be quite wrong to regard the relaxation theory as a demonstrated consequence of any of the general theories of physics. Rather, it consists in a simple phenomenological analogy, motivated indeed by ideas concerned with molecular structure, but in no sense a molecular theory.

In liquids the simple argument concerning internal degrees of freedom is no longer valid, ${ }^{34}$ yet absorption and dispersion seem to follow patterns similar to those for gases. This has been taken as evidence that the relaxation theory is still valid when the physical arguments giving rise to it are not. The theory has been shown to follow quite generally from irreversible thermodynamics without any specific statement regarding the molecules, but only at the cost of drastic mathematical assumptions about hypothetical "inner transformations." Thus the relaxation theory is no less phenomenological than the classical hydrodynamical treatment: in fact, it is no more than a special case of the phenomenological theory of flow of reacting mixtures.

Consequently, we cannot accept the relaxation theory a priori-we must carefully examine its consequences and determine how they differ from those of the hydrodynamical theory. To this end we have no need to go into the physical literature ${ }^{35}$ much of which is concerned with the evaluation of the constants in

${ }^{34} C f$. $\$ 5$ of Frenkel \& Obrastzov, op. cit. Note 56 below.

${ }^{35}$ H. O. KNEser, "Zur Dispersionstheorie des Schalles," Ann. der Phys. (5) 11, 761-776 (1931).

P. S. H. Henry, "The energy exchanges between molecules," Proc. Cambr. Phil. Soc. 28, 249-255 (1932).

H. O. Kneser, "Schallabsorption in mehratomigen Gasen," Annalen der Phys. (5) 16, 337-349 (1933).

A. J. Rutgers, "Zur Dispersionstheorie des Schalles," ibid. 350-359.

H. O. KNESER, "The interpretation of the anomalous sound absorption in air and oxygen in terms of molecular collisions," J. Acoust. Soc. Am. 5, 122-126 (1933).

Y. Rocard, "Sur l'absorption quantique du son dans les gaz," C. R. Acad. Sci. Paris 
terms of molecular or thermodynamical properties, the generalization to the case of several processes, alternative derivations, etc. We shall rest content with the formal aspects of the theory for a single relaxation process, and to this end it suffices to consider Einstein's treatment. We shall sometimes refer to the relaxation theory as the two fluid model, to distinguish it from the visco-thermal or one fluid model.

First, we must replace Einstein's approximation (4.26) by the exact dispersion following from $(4.25)_{1}$, viz.

$$
r=\frac{1}{\bar{r}} \frac{2\left(1+\bar{X}^{2}\right)}{1+K^{2} \bar{X}^{2}+\sqrt{1+K^{4} \bar{X}^{2}} \sqrt{1+\bar{X}^{2}}} .
$$

When $0<K<1$ the approximation (4.26) is too large except at $\bar{X}=0$ and $\bar{X}=\infty$; in order that it be tolerable throughout the whole frequency range it is necessary and sufficient that $1-K \ll 1+K$; but as $K \rightarrow 0$ the error grows larger, and finally for $K=0$ (4.26) gives dispersion which is too great by a half order of magnitude as $\bar{X} \rightarrow \infty$.

Comparison of $(4.25)_{2}$ and (4.27) with (4.6) yields the

Identification theorem. ${ }^{36}$ Einstein's two fluid ("relaxation") model and Stokes's radiation theory for a pure fluid yield identical absorption and dispersion, except for physical interpretation of constants. The identification is achieved through the substitutions $\bar{X}=\gamma S^{\prime}, K^{2}=\gamma^{-1}, \bar{r}=\gamma$. This result becomes physically

198, 802-803 (1934). See also pp. 54-62 and Ch. II, $\$ \$ 7-8$, respectively, of the first two works cited in Note 47 below.

H. O. Kneser \& O. Gauler, "Das Problem der Schallausbreitung in teilweise dissoziierten Gasen," Phys. Z. 37, 617-684 (1936).

Л. И. Мандельштам и М. А. Леонтович, “К теории поглощения звука в жидкостях," अЖ. Експ. Теор. Физ. 7, 438-449 (1937).

H. O. KNEsER, "Schallabsorption und -dispersion in Flüßigkeiten," Ann. der Phys. (5) 32, 277-289 (1938).

K. SchÄFER, "Die Stoßanregung intramolekularer Schwingungen in Gasen und Gasmischungen. VIII. Theorie der Schalldispersion bei Vorhandensein mehrerer Normalschwingungen," Z. Physikal. Chemie B46, 212-228 (1940).

G. DAMköhler, "Isentropische Zustandsänderung in dissoziierenden Gasen und die Methode der Schalldispersion zur Untersuchung sehr schneller homogener Gasreaktionen," Z. Elecktrochemie 48, 62-82, 116-131 (1942).

J. Merxner, "Absorption und Dispersion des Schalles in Gasen mit chemisch reagierenden und anregbaren Komponenten, I," Ann. der Phys. (5) 43, 470-487 (1943).

H. O. KNEsER, "Allgemeine theorie der Schallabsorption in Gasen und Flüßigkeiten," Nuovo Cim. (9) 7 Suppl. N. 2 (1950).

J. Meixner, "Zur thermodynamischen Theorie der Schallabsorption und Druckviskosität in homogenen Medien," Het Colloquium over Ultrasonore Trillingen, 107-112, with remark of Prigogine, 113-116 (1951).

J. MeIXNER, "Strömungen von fluiden Medien mit inneren Umwandlungen und Druckviskosität," $Z$. Phys. 131, 456-469 (1952).

A survey of the relaxation theory is given by H. O. KNESER, "Molekulare Schallabsorption und -dispersion," Ergebn. Exakten Naturwiss. 22, 121-185 (1949).

36 This result was noted apparently for the first time, though only for $r$ in the approximate form (4.26), by Markham, Beyer, \& Lindsay, op. cit. Note 27 (see \$6). 
evident when one recalls that in both theories an effective heat source distribution proportional to a small temperature difference is assumed.

Henceforth put $\bar{r}=1$. Now let $K$ approach $^{37} 0$. Then the functional forms of $(4.25)_{2}$ and (4.27) reduce precisely to those of $(4.10)_{2}$ and $(4.10)_{1}$. Hence we have the

Inclusion theorem. Except for physical interpretation of constants, the Einstein relaxation measures include as the special case $K=0$ those of Stefan derived from the theory of pure viscous piezotropic fluids.

To consider the nature of the limit $K \rightarrow 0$, we first apply the identification theorem to (4.7) and (4.8), thus obtaining the following exact results for the relaxation theory:

$$
\begin{gathered}
\bar{X}^{*}=1<\bar{X}^{\#}=\sqrt{\frac{3+K^{2}}{1+3 K^{2}}}<\bar{X}^{\natural}=\frac{1}{K}, \\
a^{*}=\frac{1}{2} \pi\left(1-K^{2}\right)<A_{0}^{\#}=\frac{1}{2} \pi\left(1-K^{2}\right) \sqrt{\frac{2}{1+K^{2}}}<A^{\natural}=2 \pi \frac{1-K}{1+K} .
\end{gathered}
$$

As $\bar{X} \rightarrow \infty, r$ increases steadily from 1 to the limit $K^{-2}$, and thus there is a limit speed $V_{\infty}=V_{0} / K$. When $K=1$ there is no dispersion or absorption. For $K$ very near to 1 , there is almost no dispersion, and the curves of $Q, A_{0}$, and $A$ are virtually alike. As $K$ decreases, the peaks in $Q, A_{0}$, and $A$, beginning at $\bar{X}=1$ for all three, spread apart in the order named; the location of the peak in $Q$ remains fixed, but those for $A_{0}$ and $A$ move to the right; simultaneously the heights of all three peaks increase steadily. Meanwhile the maximum dispersion increases without limit. As $K \rightarrow 0$, the peak in $A_{0}$ reaches its limit position $\bar{X} \#=$ $\sqrt{3}$, its height approaches $\frac{1}{2} \pi \sqrt{2}$, while the height of the peak in $a$ reaches $\frac{1}{2} \pi$. But, while its height increases to $2 \pi$, the peak in $A$ moves out to $\infty$. Simultaneously the asymptote $r=K^{-2}$ for the dispersion curve moves to $\infty$, as does also the point of inflection.

A corollary to the above discussion is the

Viscosity bound theorem for relaxation absorption. The maximum absorption coefficient given by the Einstein relaxation theory for $K \neq 0$ is never so great as the maximum of the corresponding coefficient for a pure viscous piezotropic fluid.

This theorem would at first seem to give a very odd ring to the common statement that a relaxation mechanism is necessary in order to yield absorption as great as that which is experimentally measured, since it shows that we must suppose relaxational dispersion to be very large indeed in order to get maximum absorption even approximately as large as that afforded by viscosity alone. But in all our comparisons so far we have discussed properties independent of the frequency scale. $X$ and $\bar{X}$, however, are not alike. Put $k=\bar{X} / X$. By changing

${ }^{37}$ There are various ways of achieving this process in the interpretation. In EInstern's notations, hold $\left(K^{2} \bar{C}\right) /\left(\kappa_{1} B\right)$ finite and let $\bar{C}$ approach 0 . Tn KNESER's notations, suppose $C_{i} \gg C_{a}$. 
$k$ we can move the relaxation peak for $A_{0}$ (or $Q$ ) to any position we desire relative to the viscosity peak, simultaneously giving an arbitrarily large value to the initial slope of the curve of $A_{0}$ against $\omega$. When Einstein wrote that he was neglecting viscosity altogether, he in effect assumed $k=\infty$, putting the viscosity peak at $\infty$, while the peak for dissociation (i.e. relaxation) was supposed to occur at a relatively low frequency. ${ }^{38}$ When $k$ is either very large or very small, the relaxation absorption coefficient will be large if and only if the viscosity absorption coefficient be very small, and conversely. Under these circumstances it may

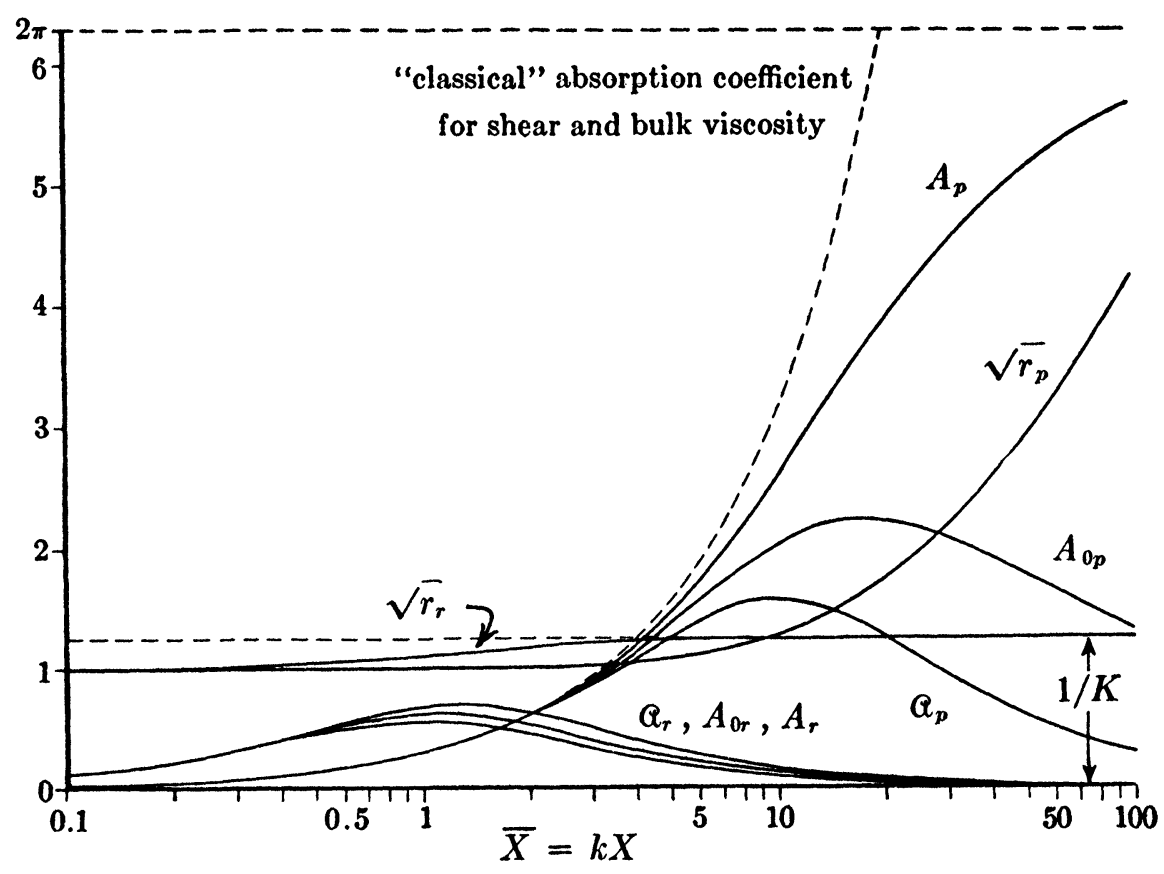

Fig. 4.3

Absorption and dispersion measures for relaxation and for viscosity, drawn to the same scale, for the case $k=10, K=\frac{1}{5}$

be permissible to superpose the two effects, as is commonly done in the experimental literature. Plainly, however, it is absolutely wrong to superpose the results of the viscosity and relaxation theories when the former alone accounts for a sizeable amount of the measured absorption. (See the end of §5.) For a spe-

38 Note that if STOKEs's and RAYLEIGH's argument that $S^{\prime} \gg 1$ at audible frequencies be accepted, from (4.6) it must follow that at subaudible frequencies there are sharp and relatively high peaks in $Q, A$, and $A_{0}$, of magnitude given by (4.8). When $\gamma=1.4$, the height of the peak in $A_{0}$ is $.18 \pi$. Most of the relaxation literature assigns to $K$ a value quite near 1 in specific applications. The predicted radiation peak at subaudible frequency is higher than a relaxation peak corresponding to such a value of $K$. This radiation peak has not been noted or looked for, so far as I know. 
cial case, the measures corresponding to the relaxation and viscosity theories are shown to the same scale in Fig. 4.3. Note the logarithmic frequency scale: had a linear scale been used for $0 \leqq X \leqq 5$, the relaxation absorption curves would have appeared almost insignificant.

But how is the frequency scale to be set? Now in the relaxation theory it may be adjusted by a disposable constant, $\bar{X} / \omega$, essentially the "relaxation time." Thus the peak in any one of the absorption measures may be put at as low a frequency as desired. But in the viscosity theory also there remains the unspecified parameter $\mathcal{V}$, by adjusting which it is possible to make the frequency scales of the viscosity and relaxation theories identical. Hence no distinction in results between the two theories is possible on the basis of the location of the absorption peak. The magnitude of the relaxation peaks, however, being given by (4.28), depends upon the constant $K^{2}$, whose reciprocal is the maximum dispersion. When $K$ is small, the difference between the two theories disappears, but when $K$ is near to 1 the predictions become quite different except at the lowest frequencies. Hence we have the following

Dispersion criterion. If the second viscosity $\lambda$ may be regarded as a disposable constant, then it is only in dispersion and in the effects of dispersion that the relaxation theory will yield results essentially different from those of its special case $K=0$, the pure viscosity theory. ${ }^{39}$

Consider now the effect of dispersion. The coefficient $Q$ is independent of it, and, apart from a constant factor, is exactly the same as $Q_{p}$. But the extremely strong effect on the true absorption coefficient $A$ is easiest seen by elimination of $\bar{X}$ from $(4.25)_{1}$ and (4.27):

$$
\frac{\left[\frac{A /(2 \pi)}{1-A^{2} /\left(4 \pi^{2}\right)}\right]^{2}}{\left[\frac{1-K^{2}}{4 K}\right]^{2}}+\frac{\left[\frac{r}{1-A^{2} /\left(4 \pi^{2}\right)}-\frac{K^{2}+1}{2 K^{2}}\right]^{2}}{\left[\frac{1-K^{2}}{2 K^{2}}\right]^{2}}=1 .
$$

Interpretation of this identity yields the elegant

Kneser semi-ellipse theorem. ${ }^{40}$ The curve of $[A / 2 \pi] /\left[1-A^{2} /\left(4 \pi^{2}\right)\right]$ against $r /\left[1-A^{2} /\left(4 \pi^{2}\right)\right]$ is a semi-ellipse with left vertex $(0,1)$, center $\left(0,\left(K^{2}+1\right) /\left(2 K^{2}\right)\right)$, vertical semi-axis of length $a=\frac{1}{4}\left(1-K^{2}\right) / K$, horizontal semi-axis of length $\frac{1}{2} a K$. When $K$ is near to 1 , the semi-ellipse is approximately a small semi-circle. As $K \rightarrow 0$, the center and the right vertex move out to the right, as does the upper vertex, all magnitudes increasing without limit. For $K=0(4.29)$ is to be re-

${ }^{39}$ Cf. Herzfeld \& Rice, op. cit. Note 33, "A real test of the theory can be made only if for the same gas absorption and dispersion are measured carefully."

${ }^{40}$ The statement in the text is an easy generalization of the special case valid for $K \approx 1$ given in KNESER's paper "Über den Zusammenhang zwischen Schallgeschwindigkeit und -absorption bei der akustischen Relaxation," Ann. der Phys. (5) 43, 465-469 (1943). (The misprints in eqq. (1) (2) (3) are corrected in the Ergebnisheft cited in Note 35). KNESER plots $A$ against $\pi K r$, thus obtaining a semi-circle. 
placed by

$$
r=\frac{\left(1+\frac{A^{2}}{4 \pi^{2}}\right)^{2}}{1-\frac{A^{2}}{4 \pi^{2}}}
$$

These results are shown graphically in Fig. 4.4.

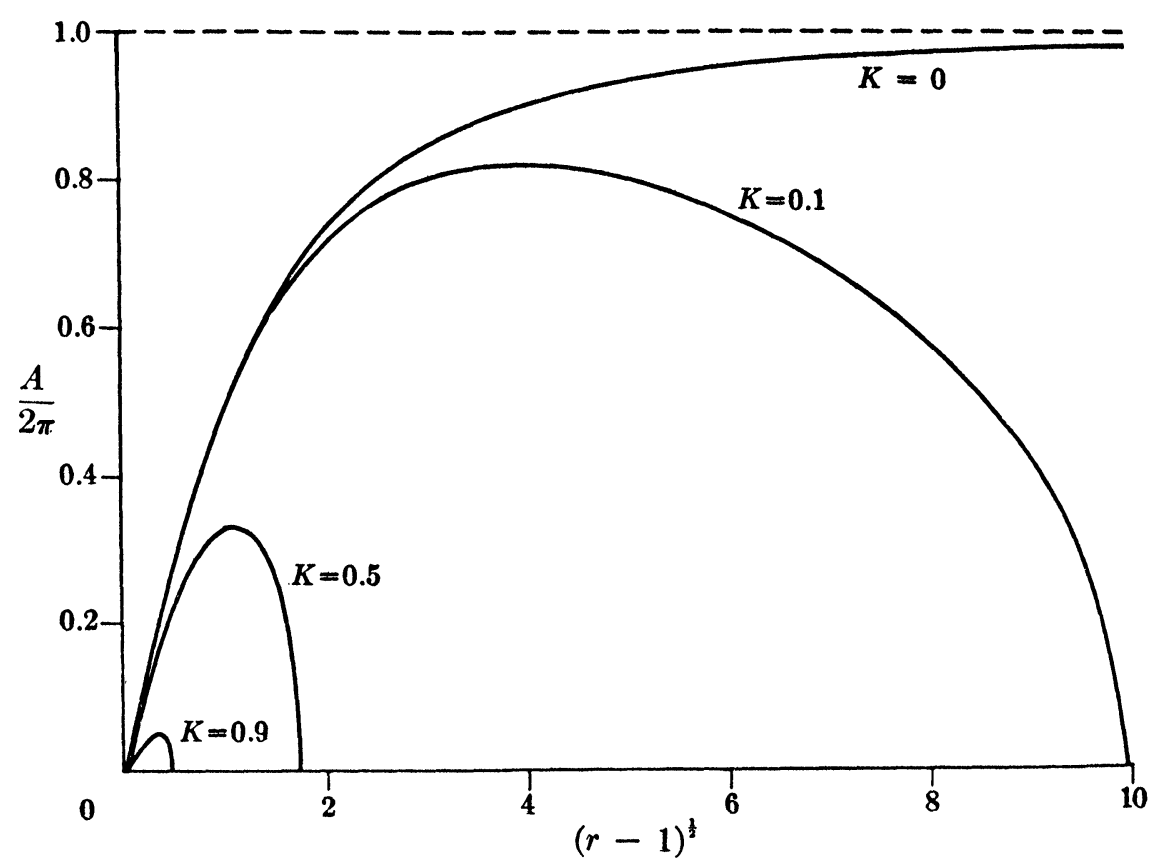

Fig. 4.4

Absorption as a function of dispersion, according to the relaxation theory

That Kneser ${ }^{41}$ has found for several polyatomic gases very good agreement with experiment for the semi-circular approximation is evidence that Stefan's formulae are inadequate. Whether or not results in better agreement with experiment can be obtained from the exact solutions of the visco-thermal theory remains an open question. The prospect, however, is poor, since, as we have seen, according to that theory $r \rightarrow \infty$ as $X \rightarrow \infty$, while the Kneser semi-circle approximation is possible only when $r$ approaches a limit value very close to 1 .

Before leaving the relaxation theory we must note that van Itterbeck \& Mariëns ${ }^{42}$ interpreted their measurements of absorption peaks at various pressures as show-

41 Ergebnisheft, Abb. 15.

42 "Measurements on the absorption of sound in carbon dioxide gas. Determination of the relaxation time for the vibrational energy as a function of the temperature," Physica 5, 153-160 (1938). 
ing that for a given fluid, $\tau$ is inversely proportional to pressure, a result since generally accepted. The relaxation theory in itself gives us no reason to expect any particular thermodynamic dependence for $\tau$, and a plausibility argument had to be constructed a posteriori. For perfect gases, the hydrodynamical formulae (4.14) predict precisely this dependence.

\section{Other proposals.}

The effect of the structure of gas molecules on the passage of waves was thoroughly investigated in a series of remarkable papers by D. G. Bourgin. ${ }^{43}$ They follow not the kinetic theory approach initiated by Boltzmann and Lorentz, but rather the method of small perturbations from statistical equilibrium. The general results are expressed in terms of infinite series of molecular properties and are difficult to analyse qualitatively or to interpret physically. Bourgin has taken great pains, however, to find what additional simplifications are necessary to reduce them to the relaxation theory or its immediate generalizations. This analysis remains to the present day the strongest theoretical argument for the relaxation theory in gases. Since, however, it takes no account of transport processes in a streaming gas, it cannot be considered a final treatment. The question remains open both as to whether the effects taken into account by Bourgin predominate over those neglected, and whether the results obtained constitute in whole or in part a molecular explanation for terms in the hydrodynamical equations, or imply the need for modifying those equations themselves.

$\mathrm{Y}$. Rocard ${ }^{44}$ reviewed the hydrodynamical theory of absorption, neglecting to use an independent second viscosity. He initiated the unfortunate custom of treating the effects of viscosity, heat conduction, and radiation singly, then superposing the results. Such a method is valid only at the lowest frequencies. Rocard was the first to calculate the absorption arising from diffusion in a mixture, using a form of the energy equation derived earlier by Chapman from the kinetic theory. For air at low frequencies he calculated that absorption from uhis cause should be about one sixth of that from shear viscosity.

The hydrodynamical equations themselves, of course, are only a first approximation. Whether viewed from a molecular model or from a general definition of a fluid as a continuum possessed of a certain class of responses, the stresses and heat flux in higher approximation depend upon pressure, temperature, and velocity gradients of all orders and upon arbitrarily high powers of these quantities. ${ }^{45}$ The terms of the latter type have no effect on the propagation of infinitesi-

\footnotetext{
${ }_{43}$ "Kinetics of absorption of ultra-sonic waves," Nature 122, 133 (1928).

"The propagation of sound in gases," Phil. Mag. (7) 7, 821-841 (1929).

"Sound propagation in gas mixtures," Phys. Rev. (2) 34, 521-526 (1929).

"The velocity of sound in an absorptive gas," Phys. Rev. (2) 42, 721-730 (1932).

"Sound absorption in non-reactive gas mixtures," J. Acoust. Soc. Am. 5, 57-59 (1933).

"Sound absorption and velocity in mixtures," Phys. Rev. (2) 50, 355-369 (1936).

44 "Sur l'amortissement des ondes sonores dans un milieu gazeux homogène," $J$. Phys. Rad. (7) 1, 426-437 (1930).

45 A survey of both viewpoints given in $\mathrm{Ch}$. $\mathrm{V}$ of the first of my papers cited in Note 12.
} 
mal waves, although their magnitude may well have a bearing on the range of amplitudes which lends itself to approximation by the theory of small oscillations. The former, however, contribute to the absorption and dispersion measures. Rocard $^{46}$ was the first to consider the effect of some of the terms involving density gradients. Deriving values for the coefficients from the kinetic theory of gases, he concluded that these particular terms give rise of themselves to no absorption, and to but a slight anomalous dispersion: "la correction est negligeable, elle compense encore toutefois simplement la diminution de vitesse du son due à la viscosité, par exemple, ou à la conductibilité thermique."

In later works Rocard ${ }^{47}$ gave what he emphasized to be rigorous values for the absorption coefficient and the dispersion caused by viscosity. These values do not agree with (4.10); in particular, Rocard predicted normal rather than anomalous dispersion. No derivation is supplied, and I have not been able to find any alternative explanation which could render these results correct. For a conducting but non-viscous fluid Rocard derived the next term after (4.22) in the power series expansion for $A_{0}$.

R. Lucas ${ }^{48}$ derived Stefan's formula $(4.10)_{1}$ for dispersion, but he contended that for absorption certain thermal fluctuations of density need to be considered. The result of his phenomenological treatment of this effect is equivalent to choosing a value of $v$ slightly greater than $\frac{4}{3}$ in Kirchhoff's result (4.20), an amount he stated to be insufficient. He included both $\lambda$ and $\mu$ in his theoretical formulae, but in comparison with experiment he always set $3 \lambda+2 \mu=0$. Later $^{49}$ he derived Stefan's formula for absorption, noting the first approximation for $r$ and $A_{0}$ as $X \rightarrow \infty$, but setting $V=\frac{4}{3}$ throughout. These results caused him to retract in part his earlier conclusion that the hydrodynamical theory is insufficient.

The relaxation theory was created ad hoc to explain ultrasonic absorption and dispersion; while indeed for gases it is based on a certain physical idea, it is isolated from mechanics in general. It gives no idea how the fluid in question would behave in any circumstance other than infinitesimal oscillation. The response predicted by the relaxation theory, if it really exists and is not described by hydrodynamics, indicates that fluids have some purely mechanical properties

46 "Equations hydrodynamiques avec termes capillaires. Théorie de la tension superficielle," J. Phys. Rad. (7) 4, 533-548 (1933). See $\$ 4$.

47 "'Sur l'absorption du son dans les gaz notamment aux fréquences très élevées," Rev. d'Acoust. 3, 47-62 (1934); "Propagation et absorption du son," Act. Sci. Ind. 222, Paris (1935). See pp. 48-49 of the former; Ch. II, $\$ 2$ of the latter. At the end of the former of these. ROCARD retracted another tentative explanation he had given in "L'absorption du son dans l'atmosphère: une tentative d'explication," $J$. Phys. Rad. (7) 1, $426-437$ (1930).

48 "Sur la propagation des ultrasons dans les milieux liquides," C. R. Acad. Sci. Paris 201, 1172-1174 (1935).

"Sur l'absorption des ondes élastiques dans les fluides," ibid. 203, 459-461 (1936).

"Absorption et dispersion des ultrasons et structures des liquides," J. Phys. Radium (7) 8, 41-48 (1937).

49 "Sur les ondes longitudinales de fréquences très élevées dans les fluides visqueux," C. R. Acad. Sci. Paris 206, 658-659 (1938). 
not included in the one fluid hydrodynamical model. Such properties should manifest themselves not in ultrasonics alone, but in a variety of mechanical situations.

This new viewpoint was first brought to the subject-it must be confessed, rather obscurely-by A. Gemant," who suggested that fluids exhibiting "relaxation" effects are in fact visco-elastic substances.

Mandelstam \& Leontovic ${ }^{51}$ were the first authors since the rise of ultrasonics to recall the full form (4.20) of Kirchhoff's result, both viscosities included. They stated that sound absorption measurements in liquids indicated large values of $v$, mentioning about 90 for benzene, and that consequently it is $\lambda$ rather than $\mu$ or $\kappa$ which gives rise to the major part of the absorption of sound in most liquids. They stated also that the linear approximation to the relaxation absorption coefficient $(4.25)_{2}$ could be interpreted as yielding a value of $v$ from the relaxation theory. If we put ${ }^{52} \bar{X}=h S$, their statement is equivalent to

$$
v=\frac{4}{3}+h\left(1-K^{2}\right) \text {. }
$$

Their view is reasonable, as far as gases are concerned. The phenomenological theory of viscous fluids yields two independent viscosities, say $\lambda+\frac{2}{3} \mu$ and $\mu$; in the molecular model of a gas, linear momentum transfer alone yields a molecular mechanism for $\mu$, while intramolecular momentum transfer yields a molecular mechanism for $\lambda+\frac{2}{3} \mu$. The results derived by Bourgin from statistical mechanics may be interpreted most reasonably in this way, within the range of linear absorption. It is not correct, however, to assume that $\mu$ is unaffected by internal degrees of freedom or that $\lambda+\frac{2}{3} \mu$ is unaffected by translatory molecular motion; even less correct is it to try to apply these ideas to liquids.

Since the paper of Mandelstam \& Leontovič several further molecular mechanisms for the second viscosity have been proposed. To follow them would lead us afield from our subject, ${ }^{53}$ which is confined to non-linear gross phenomena.

In the same year Leontovich ${ }^{54}$ first advanced an argument which has since become familiar. The hydrodynamical equations have been derived from the kinetic theory of gases as the leading terms in certain series expansions in powers

\footnotetext{
so "Komplexe Viscosität," Die Naturwiss. 23, 406-407 (1935).

"Compressional waves in media with complex viscosity," Physics 6, 363-365 (1935).

"The conception of a complex viscosity and its application to dielectrics," Trans. Faraday Soc. 31, 1582-1590 (1935).

s1 "Über die Ultraschallabsorption in Flüßigkeiten und über einige damit zusammenhangende optische Erscheinungen," C. R. Doklady Acad. Sci. SSR. $12=(2)$ 3, 111-114 (1936).

52 In KNESER's notations (Note 32), $h=\tau^{\prime} \rho V_{0}^{2} / \mu$. A similar conclusion was reached from somewhat more general grounds by Merxner, op. 1943 cit. ante, Note 35.

${ }^{53}$ Some of them are discussed in my paper in $Z$. Phys. cited in Note 12 and in $\$ 61 \mathrm{~A}$ of my paper in J. Rat. Mech. Anal. cited in the same note.

${ }_{64}^{4}$ М. А. Леонтович, "Некоторые вопросы теории поглодения ввука в многоатомных газах", Изв. Акад. Наук СССР Отдел. Мат. Ест. 1936, 633-642.
} 
of a parameter which in the case of infinitesimal oscillations reduces to (mean free path/wave length). "Thus we can see clearly in advance that the hydrodynamical theory can be valid only if this ratio is sufficiently small." He claimed further that the hydrodynamical equations can yield valid results only as far as the Kirchhoff approximation (4.20), while the relaxation theory has a somewhat greater range of correctness. He asserted that Hilbert's method of integrating the Boltzmann equation of the kinetic theory of gases can be extended to the polyatomic case, for which a non-vanishing bulk viscosity results. It is impossible to tell whether he was reporting work actually done or merely expressing his confidence in the outcome of calculations yet to be performed. He stated that according to unpublished work of Drabkin a modification of Hilbert's method yields Kneser's form of the relaxation theory as a consequence of the Boltzmann equation. Finally, he asserted that analogous results hold for liquids, but he did not reveal how he had come to this opinion.

Issakovich $^{55}$ found that results similar to those of the relaxation theory could follow from a theory of visco-elasticity different from that employed by Gemant.

In order to represent the continuity of the liquid and solid state, Frenkel \& Obrastzov $^{56}$ set up equations of a more general visco-elastic model exhibiting elasticity, elastic hysteresis, viscosity, etc., specified through seven material constants. The formulae for absorption and dispersion obtained from this theory are equivalent to a superposition of Stefan's non-linear formulae for the effect of viscosity upon Einstein's non-linear formulae for the effect of "relaxation," each containing additional dimensionless constants at various points. This result includes those of Gemant and Issakovich.

The first attempt to combine the relaxation theory rationally with ordinary hydrodynamics was made by $\mathrm{Z}$. Sakadi. ${ }^{57}$ In addition to the equations for a reacting mixture of two components, he employed the ordinary hydrodynamical formulae for viscous stress and heat flux. The resulting theory is extremely complicated. He noted that any amount of viscosity, however small, suffices ultimately to destroy the finite limit property for the speed of sound which characterizes the relaxation theory in all its forms. He noted the difficulty of calculation for general frequency, but was not able to advance beyond these qualitative remarks.

65 "Propagation of waves in a liquid possessing Maxwell viscosity," C. R. (Doklady) Akad. Sci. URSS 23, 783-787 (1939).

56 "A phenomenological theory of the mechanics of amorphous bodies," J. Physics Acad. Sci. USSR 2, 131-142 (1940) (Russian version in ЖK. Експ. Теор. Физ. 9, 1081-1093 (1939).) Cf. J. Frenkel, Kinetic Theory of Liquids, Oxford (1946), see Ch. IV, \$\$7-8, 10. Cf. also I. Osida, "On the mechanical behavior of liquids under high frequeney oscillation," Proc. Physico-Math. Soc. Japan (3) 23, 18-27 (1941), and "On the theory of the mechanical properties of liquids in the supersonic and hypersonic region," Mem. Fac. Eng. Nagoya 2, 2950 (1950).

57 "On the dispersion of sound wave considering the effects of heat conduction and viscosity," Proc. Phys.-Math. Soc. Japan (3) 23, 208-213 (1941). See also J. Meixner, "Allgemeine theorie der Schallabsorption in Gasen und Flüßigkeiten unter Berücksichtigung der Transporterscheinungen," Acustica 2, 101-109 (1952). 
In an influential paper L. Tisza ${ }^{58}$ stated it to be "an unquestioned fact that there is a time lag in the establishment of equilibrium for the internal degrees of freedom and consequently a dissipation of energy," but ". . . the classical hydrodynamical equations can easily be generalized so as to include this phenomenon." He began by repeating Mandelstam \& Leontovič's identification of the total relaxational absorption at low frequencies with the total viscous absorption. According to this view, all that is necessary is to adjust $v$ so that the initial slope of the absorption curve equals the measured value. If we denote by $A_{c}$ the "classical" value (4.21), then when $S \ll V^{-1}$ we get by $(4.20)$

$$
v=\frac{4}{3}\left[1+\left(1+\frac{3(\gamma-1)}{4 P}\right)\left(\frac{A}{A_{c}}-1\right)\right],
$$

in which we may replace $A / A_{C}$ by the corresponding ratio $\alpha / \alpha_{C}$ of absorption coefficients per $\mathrm{cm}$ if we please. From absorption data Tisza thus concluded that $v \approx 2 \times 10^{3}$ in carbon dioxide and in nitric oxide.

Tisza mentioned also that one can introduce formally a complex and frequency dependent $\lambda$, by selecting whose form appropriately one can get full agreement at all frequencies between the hydrodynamical theory and the relaxation theory. This remark has sometimes been misunderstood, and a "frequency dependent bulk viscosity" has marred the literature. The very nature of the two viscosities $\lambda$ and $\mu$ in (2.6) implies that they are independent of whatever deformation may take place: otherwise the linearity of (2.6) would be meaningless. If $\lambda$ and $\mu$ are allowed to depend on $\omega$ in plane wave propagation, what is their functional form for general deformation? And why should it be only $\lambda+\frac{2}{3} \mu$ which is allowed to be frequency dependent, rather than both $\lambda$ and $\mu$ ? The same result could be forced by retaining the Stokes relation but permitting $\mu$ to depend upon $\omega$ in a suitable way. What physical meaning can a complex viscosity coefficient have? Furthermore, we note that Tisza first linearizes the hydrodynamical absorption formula with respect to $\omega$, then inserts ${ }^{59}$ in it a $\lambda+\frac{2}{3} \mu$ which depends non-linearly upon $\omega$. By such Procrustean hospitality almost any two theories can be racked into agreement. The whole virtue of the hydrodynamical theory is that it predicts absorption and dispersion measures, not that it can be altered to fit data. However, formulae similar to that proposed by Tisza, while impossible within the classical theory of viscous fluids, do indeed result from the visco-elastic theories of Gemant, Issakovich, Frenkel \& Obrastzov, and Osida, which when applied to plane infinitesimal waves yield an equation identical in form with the wave equation of classical elasticity theory, except that the elastic

58 "Supersonic absorption and Stokes' viscosity relation," Phys. Rev. (2) 61, 531-536 (1952). On p. 532, col. 1, he appears to claim that all fluids are piezotropic, and in col. 2 to retract this statement.

${ }^{59}$ Such also is the method of H. KNeser, "Druck- und Schubviskosität in Gasen," Annalen der Phys. (6) 6, 253-256 (1949). The supposed derivations of frequency dependent bulk viscosity from irreversible thermodynamics (e.g. MerXner, op. 1951 cit. Note 35) at bottom reduce to such pure formalism. 
moduli are replaced by certain functions of $i \omega$. To the student of mechanics, the introduction of a "complex viscosity" can appear as nothing but a rather obscure way of stating that the physical fluid is to be regarded as a visco-elastic bodya proposal which, as we shall see in $\$ 5$, is not very attractive.

Consideration of the effect of higher order terms in the hydrodynamical equations, initiated by Rocard, was carried further by H. Primakoff, ${ }^{60}$ who used the expressions for the stress and heat flux derived by Burnett and Chapman \& Cowling from approximate integration of the Boltzmann equations of the kinetic theory of gases. Essentially the same analysis was repeated by H.-S. Tsien \& R. Schamberg, ${ }^{61}$ who carried power series expansions for $r$ and $A_{0}$ as far as terms of order $S^{6}$ and $S^{5}$, respectively. Numerical values of the various coefficients appropriate to special molecular models were employed. While the results of both these calculations indicate very slight dispersion over the range considered $\left(S<\frac{1}{4}\right)$, Primakoff found this dispersion to be normal, while Tsien \& Schamberg found it to be anomalous. The numerical conclusions of these authors have had to be modified (see below). Tsien \& Schamberg stated that the Burnett and Chapman \& Cowling formulae are correct only to order $O\left(S^{2}\right)$, hence claiming that the consequent results are to be treated only to the same order. They also hinted at difficulty in the convergence of their solutions near $S=\frac{1}{2}$.

From the analysis of Frenkel \& Obrastzov and of various writers on irreversible thermodynamics there arose a deplorable notion that a "modified equation of state" may be "written down by inspection." "61a According to this view, one has only to replace the usual static proportionality between excess pressure and excess density by a similar proportionality between linear combinations of these with various of their time derivatives. While indeed such a relation emerges as the outcome of a phenomenological treatment, to construct it from thin air is to beg the whole question of the physical nature of absorption and dispersion: one might as well write down the end forms of the absorption and dispersion relations "by inspection."

In 1945 an experimenter first stated that by measuring the absorption coefficient he had measured the number $v$. For water at $20^{\circ} \mathrm{C} \mathrm{E}$. Hsu ${ }^{62}$ obtained $v=3.6$.

In a work published only in abstract C. Eckart ${ }^{63}$ wrote "In discussing the

co "The translational dispersion of sound in gases," J. Acoust. Soc. Am. 13, 14-18 (1942).

61 "Propagation of plane sound waves in rarefied gases," $J$. Acoust. Soc. Am. 18, 334341 (1946).

61a KNEser, op. cit. Note 40 et op. 1950 cit. Note 35; R. B. Lindsay, "Transmission of sound through air at low pressure," Am. J. Phys. 16, 371-377 (1948); MArkham, BeYer, \& LINDSAY, op. cit. Note 27.

62 "Measurement of supersonic absorption in water by the balance method with mechanical integration," J. Acoust. Soc. Am. 17, 127-131 (1945). F. E. Fox \& G. D. Rock, "Compressional viscosity and sound absorption in water at different temperatures," Phys. Rev. (2) 70, 68-73 (1946), at the suggestion of E. TELLER similarly presented their results in terms of $\mathcal{V}$.

${ }_{63}$ "Viscous and thermal absorption of sound as relaxation phemonema," Phys. Rev. (2) 71, 277 (1947). 
absorption of sound, reference is often made to relaxation phenomena, as distinguished from the classical viscous and thermal dissipative processes. While this may be convenient, it is not a basic distinction. By using known formulae, it is shown that viscous dissipation of sound can be considered as a relaxation phenomenon, with relaxation time $\tau_{\nu}=4\left(\nu+\nu^{\prime}\right) / 3 \rho c^{2}$, where $\nu, \nu^{\prime}=$ ordinary and dilational coefficients of viscosity, $\rho=$ density of fluid, and $c=$ velocity of sound. Similarly, the relaxation time for thermal dissipation is $\tau_{\kappa}=\kappa / \rho c^{2} S$, where $\kappa=$ thermal conductivity and $S=$ specific heat at constant volume." A similar view was expressed by Lindsay, ${ }^{64}$ who added for heat conduction the qualifying phrase, "to a first approximation." For viscosity he gives $\tau=4 \mu / 3 \rho V_{0}^{2}$, for heat conduction $\tau=(\gamma-1) \kappa / \gamma c_{v} \rho V_{0}^{2}$. Now what is meant by a "relaxation process" is not clear. After exploring various conjectures in vain, I surmise that Eckart simply writes the dimensionless frequency variable in the form $\tau \omega$; for viscosity alone, $X=\tau_{\nu} \omega$ while for heat conduction alone $X Y=\tau_{k} \omega$. This conjecture similarly explains the former statement of Lindsay, but not the latter.

S. B. Gurevich ${ }^{65}$ stated that in place of the relaxation theory it is sufficient to use the exact solutions of the linearized hydrodynamical equations, due care being taken to include the second viscosity. To this end he derived anew Stefan's formulae (4.10). He noted that the predicted decrease in $A_{0} / X$ with increasing frequency is indeed observed in very viscous fluids. He remarked that it was neglect of the independence of the second viscosity, combined with linearization of $(4.10)_{2}$, which had brought on the relaxation theory, concluding that the question at issue had been confused and that unnecessarily difficult theoretical explanations had been proposed. He noted that as $X \rightarrow \infty$ $(4.10)_{1}$ yields

$$
v^{2} \sim 2(\lambda+2 \mu) \omega / \rho
$$

so that ultimately at very high frequencies the speed of propagation is determined by the viscosity alone, independently of the form of the equation of state $p=p(\rho)$, while at low frequencies it is the latter alone which determines $V_{0}$. Thus for two piezotropic fluids in which $(\lambda+2 \mu) / \rho$ has the same value, the speeds of propagation are asymptotically equal at high frequencies, although at low frequencies they may be entirely different from one another. Finally he remarked that according to the hydrodynamical theory dispersion and non-linear absorption set in simultaneously, as had already been observed experimentally in several liquids. We may notice that the same does not hold for the relaxation theory in general, which for $K \approx 1$ predicts so little dispersion that it is usually neglected in calculating the absorption coefficient. ( $C f$. also the dispersion criterion in Part $\mathrm{C}$, above.)

${ }^{64}$ Op. cit. Note 61a.

${ }^{65}$ С. Б. Гуревич, "Поглощения ултраакустических волн в жидкостях", Доклады Акад. Hayr CCCP (2) 55, 17-20 (1947). 
The work of Gurevich was immediately attacked by Bazhulin \& Leontovich ${ }^{66}$ in harsh authoritarian terms. They flatly stated his conclusions to be wrong for two reasons. (1) If $X$ is of the order of 1 , "it is perfectly clear that it is not possible to speak of propagation of waves susceptible of the ordinary method of observation." (2) The hydrodynamical equations themselves are correct only when $X \ll 1$. Hence they concluded the relaxation theory to be unavoidable. Their first reason, however, is a mere fiat, which we are at liberty to disregard. The second will be discussed in $\$ 5$.

Wang-Chang \& Uhlenbeck ${ }^{67}$ reasserted the view of Leontovich. They claimed that if one is to get non-linear absorption or any dispersion at all, "for a consistent theory" it is necessary to resort to the Burnett equations, or to even higher order approximations from the kinetic theory, rather than the Navier-Stokes equations. To this end they corrected certain errors in Chapman \& Cowling's heat flux coefficients, thus obtaining finally for Maxwellian molecules

$$
r=1+\frac{215}{36} S^{2}, \quad A_{0}=\pi S\left[\frac{7}{3}-\frac{5155}{216} S^{2}\right],
$$

while with a special molecular model for helium

$$
r=1+\frac{230.9}{36} S^{2}
$$

These they compared with the results "which would follow from Stokes-Navier if treated as exact," viz.

$$
r=1+\frac{47}{12} S^{2}, \quad A_{0}=\pi S\left[\frac{7}{3}-\frac{1559}{216} S^{2}\right] .
$$

(In the above quaint phrase, the word "exact" is used in an extraordinary new sense, equivalent to what would usually be called "quadratic and cubic approximations, respectively.") In these formulae it has been assumed that $\gamma=\frac{5}{3}$, $P=\frac{2}{3}, \vartheta=\frac{4}{3}$; hence $Y=\frac{9}{8}$.

E. Skudrzyk, ${ }^{68}$ on the basis of the linearized expression $(4.20)_{2}$ claiming that the Navier-Stokes equations are insufficient to account for the observed absorption in fluids, extended the method of Frenkel \& Obrastzov by replacing $\lambda$ and $\mu$ by infinite series in the operator $\partial / \partial t$. Since these series contain infinitely many coefficients, any given data can be fitted, but nothing can be predicted.

In an important paper Eckart ${ }^{69}$ derived second order perturbation formulae from the Navier-Stokes equations. According to his result, the second viscosity $\lambda$ can be determined from the measured stream velocity of a fluid on the acous-

\footnotetext{
${ }^{6}$ ПI. А. Бажулин и М. А. Леонтович, "Поглошение улвтразвуковых волнвжидкостях," Доклады Акад. Наук СССР 67, 29-30 (1947).

${ }^{67} \mathrm{C}$. S. WANG-Chang \& G. E. Uhlenbeck, On the transport phenomena in rarified gases," APL/JHU/CM-443, UMH-3-F, Feb. 20, 1948; WANG CHANG, The dispersion of sound in helium, APL/JHU CM-467, UMH-3-F, May 1, 1948.

68 "Die Theorie der inneren Reibung in Gasen und Flüßigkeiten und die Schallabsorption," Acta Phys. Austr. 2, 148-181 (1948).

69 "Vortices and streams caused by sound waves," Phys. Rev. (2) 73, 68-76 (1948).
} 
tical axis of a sound beam. The great value of this work lies in its providing a means of measuring $\lambda$ independently of absorption and dispersion-experimentally independent, that is, not theoretically, since both the KirchhoffLangevin equation (4.16) and Eckart's formulae are approximate consequences of the same basic theory, namely, the ordinary hydrodynamics of viscous, compressible fluids.

The necessary experiments were performed by Liebermann. ${ }^{70}$ The values he obtained for $v$ by streaming measurements in various liquids ranged from 3 to 200. He stated that these values were in rough agreement with those obtained from absorption data; presumably, by means of (4.32). For example, his value for pure water is $V=4.4$.

At this point, the tide began to turn in favor of the hydrodynamical theory. Mikhailov \& Gurevich (1949), undismayed by the $a$ priori reproofs of Bazhulin \& Leontovich, found good agreement between Stefan's formula $(4.10)_{2}$ and experimental data on absorption in rosin; between the corollary (4.33) and measurements in another case. According to Markham, Beyer, \& Lindsay, ${ }^{71}$ "these results seem to point to a perfectly classical behavior for materials of very high viscosity." Greenspan ${ }^{72}$ reported fundamental experiments for rarefied helium, in which he carried $S$ up to values as large as 10 . He compared his measured values of $A_{0}$ with five theoretical curves: the Kirchhoff linearization (4.21), the appropriate exact (numerical) solution from the visco-thermal theory based on (4.16), Wang-Chang \& Uhlenbeck's approximations $(4.34)_{2}$ and $(4.36)_{2}$, and the exact (numerical) solution of the frequency equation from which (4.34) is derived approximately. The measured points unmistakably verify the exact visco-thermal solution. Next best, and the only other theoretical result to yield even roughly the right shape of the absorption curve, is the exact solution of the frequency equation derived from the equations of Burnett and Chapman \& Cowling, as corrected by Chang \& Uhlenbeck; next, the Kirchhoff linearization; next, the second hydrodynamical approximation; and last, by far the worst of all, the formula (4.34) $)_{2}$ which Chang \& Uhlenbeck had asserted to be correct. The failure of the approximate solutions was explained by Greenspan, who noted that the series expansion which they approximate diverges for the larger values of $S$ occurring in the experiments. Greenspan's results on dispersion similarly show the failure of the usual series method (including, of course, Mme WangChang's formulae $(4.34)_{1},(4.35)$, and $\left.(4.36)_{1}\right)$, but are about equally well fitted

\footnotetext{
70 “The bulk viscosity of liquids," Phys. Rev. (2) 73, 537 (1948).

"The origin of sound absorption in water and in sea water," J. Acoust. Soc. 20, 868-873 (1948).

“The second viscosity of liquids," Phys. Rev. (2) 75, 1415-1422; 76, 440 (1949).

${ }^{71} \mathrm{Op}$. cit. ante Note 27 , see $\$ 28$. These authors in this part of their work set $\mathcal{V}=\frac{4}{3}$ and thus do not give the hydrodynamical theory a fair trial. $C f$. their discussion of castor oil with the fact that by $(4.14)_{2}$ increase in $V$ decreases the frequency at which the maximum of $A_{0}$ occurs.

72 "Attenuation of sound in rarefied helium," Phys. Rev. (2) 72, 197-198 (1949).

"Propagation of sound in rarefied helium," J. Acoust. Soc. 22, 568-571 (1950).
} 
by the exact hydrodynamical solution and the exact solution of the frequency equation which follows from the equations of Burnett and Chapman \& Cowling. There is no indication of the point of inflection and levelling off of the dispersion curve which characterizes the relaxation theory.

Wang Chang \& Uhlenbeck in their next publication ${ }^{73}$ took note of the work of Greenspan. They chose to ignore the agreement with hydrodynamical theory, asserting that "these experiments go beyond the range of validity of the StokesNavier and Burnett approximations...," and also they recognized the objection to the series method because of its failure to converge. They continued to use it nevertheless, and after a good deal of calculation, they stated that they were unable to present a result which could be compared with Greenspan's experiments.

E. Reuss ${ }^{74}$ determined exact solutions of the frequency equation which follows from Natanson's generalization of Maxwell's theory of visco-elasticity. He concluded that the results are not in accord with experimental data.

5. The present status of ultrasonic absorption and dispersion as a problem of mechanics. There are two principal viewpoints toward theories of gross phenomena. According to the one, it suffices to predict the course of these phenomena in detail, once a minimum number of empirical constants are properly adjusted. According to the other, the phenomena themselves are of interest only in so far as they cast light upon the molecular or atomic structure of the substance in which they occur, and detailed determination of gross effects is unnecessary, provided the proper order of magnitude can be predicted. For want of better terms, we shall call the latter view physical; the former, mechanical. There is no conflict between them, and the results obtained by each complement those of the other. However, the distinction is real, and careful observance of it results in economy of thought. In this paper, as in the preceding historical survey, we are concerned solely with the mechanical view of absorption and dispersion. Consequently, we take account of molecular properties only in so far as they indicate need for different mechanical models, leaving aside entirely such questions as how bulk viscosity is to be explained as a molecular process.

There are two major subdivisions of the mechanical approach: the kinetic and the phenomenological (or continuum).

A. Kinetic theory. There is no doubt that a correct and general kinetic theory treatment of absorption and dispersion would be the best and would settle all questions. However, nearly everything concluded so far from this viewpoint is fallacious or at best unproved.

First, it may well be expected indeed that in its response to sufficiently high

${ }^{73}$ "On the propagation of sound in monatomic gases," Univ. Mich. Eng. Res. Inst., Project M999, unnumbered report, October, 1952.

${ }^{74}$ E. Reuss, "Die Stoffgleichungen hochviscoser Flüßigkeiten und ihre Anwendung auf den Ultraschall," Acta Tech. Acad. Sci. Hungar. 6, 65-78 (1953). 
frequency oscillations, a fluid may no longer be regarded justifiably as a continuum. The magnitude of "sufficiently high" remains unknown and is perhaps best determined by comparing the results of continuum theories with experiment. Those who have turned to the kinetic theory on the grounds of this objection have without fail fallen into inconsistency by thereafter deriving some sort of field theory - the relaxation theory, the Hilbert-Chapman-Enskog approximations, Grad's moment equations, etc. - for a set of field equations satisfied by observable quantities and a continuum model of matter are synonymous. It is quite likely that the Navier-Stokes equations themselves do not constitute the proper field theory for certain fluid phenomena, and that the kinetic theory can lead us ultimately to more accurate field equations for these cases. Indeed, most if not all measurements concern average properties of the molecular assembly, and thus can in principle be predicted by a suitable continuum model. In fact, this idea lies at the foundation of the kinetic theory as based upon the Boltzmann equation. Anyone who will consistently maintain that all continuum models are necessarily invalid for a certain phenomenon must also relinquish the usual mathematical formulation of the kinetic theory and revert to finite sums over molecules.

Second, grant that the assertions of Leontovich, Chang \& Uhlenbeck, etc., regarding the limited validity of the Navier-Stokes equations are correct. It by no means follows that only terms of the same low order are to be retained in the solutions of these equations. That a term which is small in an equation can have a large or even dominant effect on the solution of the equation is too well known in mathematics to require examples here. We shall be content to remark that there is no evidence that two approximations are better than one; rather the reverse: most, if not all results obtained by perturbation procedures in fluid dynamics are dubious. The notion prevalent in certain circles that for "consistency" one must in solutions drop all terms of order higher than that to which the basic equations themselves are considered valid approximations to some more general ones can be no more than metaphysical.

Third, I think it is more in accord with the tradition of rational mechanics to lay down all one's assumptions at the beginning, proceding thence by strict demonstration.

Fourth, no mean free path argument can apply to liquids, for which the NavierStokes equations are equally valid, and in which absorption and dispersion of sound are not essentially different than in gases.

Fifth, an equation is often better than its derivation: that the Navier-Stokes equations have been derived as a term of a certain order in a perturbation procedure in the kinetic theory of gases is no proof at all that they cannot be derived under much weaker or different assumptions. ${ }^{1}$

\footnotetext{
${ }^{1}$ We may select an example from another domain of mechanics. It is possible to obtain the usual differential equation of the elastica from the three-dimensional infinitesimal theory of elasticity by use of various approximations, one of them being $\sin \theta \approx \theta$. If we were to apply the type of logic in favor among certain kinetic theorists, we should conclude
} 
Sixth, the Hilbert-Enskog-Chapman expansion method, on which most of the kinetic theory arguments are based, has never been shown to converge or even to be meaningful in any sense whatever. To date, the only positive indication in favor of this method is its consistency with ordinary hydrodynamics in first approximation. There is not the slightest experimental evidence that it yields results any closer to reality than does hydrodynamics. ${ }^{2}$

Seventh, the kinetic theorists do not agree upon the appropriateness of various other proposed integration methods for the Boltzmann equation. A similar situation holds with regard to the analogous problem of the structure of shock waves, where no two kinetic theorists get results in agreement with one another. Here classical hydrodynamics has survived the attacks of those who claimed it could not possibly be correct; for the shock thicknesses according to appropriate exact solutions of the Navier-Stokes equations fall in the same range as those obtained by the various approximate kinetic theory methods (see the foregoing paper by Gilbarg \& Paolucci).

Eighth, no formula derived by any means from the kinetic theory has yet satisfactorily agreed with any experimental non-linear absorption or dispersion curve. It is in the case of rarefied helium, if anywhere, that the kinetic theory should yield good results. The controversial matter of the Stokes relation does not arise. But it is precisely here that kinetic theory results so far have failed entirely while classical hydrodynamics has succeeded (see above, p. 677).

Ninth, analysis of the only two attempts ${ }^{3}$ at formal derivation of the Boltzmann equation suggests that its validity as an expression of the general principles of the kinetic theory of monatomic gases is strictly limited-limited, possibly, to a range of gas phenomena even narrower than that which is adequately described by the Navier-Stokes equations.

Perhaps when a phenomenological theory of absorption and dispersion in full agreement with experiment has been established, it can be used as a check and aid for further progress in the kinetic theory in general. ${ }^{4}$ Hence we turn now to phenomenological models.

from this fact that elastica theory is valid only for small rotations. In fact, the very reverse is true, and elastica theory is valid for large rotations, while ordinary linear elasticity is not.

2 Recall that we are speaking strictly from the mechanical viewpoint-thus we do not intend to disparage the great service done by the kinetic theory in evaluating viscosities, conductivities, etc., in terms of molecular properties.

3 See the concluding remarks in N. Bogolubov, "Kinetic equations," Journal of Physics (USSR) 10, 265-274 (1946) ; compare the last p. of J. G. KIRKwood, "The statistical mechanical theory of transport processes II. Transport in gases," J. Chem. Phys. 15, $72-76$ (1947) with the observations in $\$ 2.1$ of H. JEFFREYs, "On the relation between direct and inverse methods in statistics," Proc. R. Soc. London (A) 160, 325-348 (1937).

${ }^{4}$ Such has been the case in the field of rubber elasticity, where the complete experimental verification of the classical theory of finite elastic deformation has definitely established the inadequacy of all molecular models yet proposed for rubber. See M. C. WANG \& E. GuTH, "Statistical theory of networks of Gaussian flexible chains," J. Chem. Phys. 20, 11441157 (1952). 
B1. Hydrodynamical (visco-thermal) theory. Since the general exact solution of the Kirchhoff-Langevin equation has not been known, there has been no fair general test of the visco-thermal theory. The one special case treated, that of rarefied helium, has shown full agreement with experiment. Led on by this success of Greenspan, I undertook in the present paper to find out whether proper use of bulk viscosity and heat conduction could account for all observed absorption and dispersion in pure fluids. The result has been negative. As we shall see in $\$ 9$, the exact visco-thermal theory cannot account for most of what have been called "relaxation effects."

However, the results of Greenspan indicate that the absorption and dispersion pattern predicted by the Navier-Stokes equations exists. They nullify completely the old (and in any case rather metaphysical) arguments to the effect that high frequency oscillation invalidates the Navier-Stokes equations: rather the reverse, the typical non-linear response occasioned by viscosity and heat conduction occurs at frequencies higher than those attained in most experiments. ${ }^{5}$

B2. Pure relaxation theory. As a semi-empirical theory, with several constants adjustable to fit data, the relaxation theory appears to be successful. It is, however, a theory set up ad hoc for a single mechanical phenomenon. It does not give us any hint of the properties of waves which are not plane, of waves of finite amplitude, of shock waves, of disturbances travelling across a stream pattern, and of the myriad other mechanical phenomena for which we still have to fall back on hydrodynamics. Since the relaxation theory describes an effect not predicted by the ordinary hydrodynamics of pure fluids, it suggests that the general hydrodynamical equations should be corrected in some way, but it gives no positive indication even of the general nature of that correction (see B4, below).

B3. Visco-elastic theories. For definiteness we shall point our discussion toward the theory of Frenkel \& Obrastzov, since it contains several other visco-elastic proposals as special cases while yet remaining simple enough that specific results can be ascertained within it. First, unlike some later imitations, the theory of Frenkel \& Obrastzov is genuinely phenomenological: an ideal continuous material is clearly and explicitly defined by tensor equations which have meaning in all types of infinitesimal deformations. This theory is then applied to the special case of plane waves. Thus, in contrast to the relaxation theory, it is not merely a semi-empirical method for fitting data.

On the other hand, visco-elastic materials are well known in the mechanics of continua, ${ }^{6}$ where they have never been shown to represent the behavior of any actual material satisfactorily. Moreover, to apply such a theory to wave propagation in an ordinary gas, which in every other situation obeys the usual hydrodynamical equations without sensible error, is a dubious procedure. A proper

5 This idea, which met vigorous opposition when put forward at the Brown University symposium in 1952, has been substantiated by further unpublished experiments of GREENSPAN on polyatomic gases exhibiting "relaxation" effects at lower frequencies.

${ }^{6} \mathrm{~A}$ bibliography is given in the footnotes to $\$ 81$ of the first of the papers cited in Note 12 to $\$ 4$. 
phenomenological theory must be able, starting from simple and general hypotheses, to account for a wide range of physical phenomena with numerical moduli determined once and for all each physical material. A theory which is constructed to explain a single phenomenon, or in which the moduli are determined anew for each phenomenon occurring in the same physical substance, is merely semiempirical. In the theory of Frenkel \& Obrastzov there are so many moduli that it is difficult to reach any specific conclusions in special cases, and of the vast variety and complexity of phenomena which so elaborate a theory must certainly predict none other beyond the mere qualitative existence of elastic hysteresis has been calculated or remarked. While in the end it is possible that this theory, or one of similar complexity, will have to be accepted, a great deal of theoretical analysis and experimental test would first be necessary.

Finally, Frenkel \& Obrastzov neglect heat conduction, thus making no use of the energy equation. Such neglect is surely not justified for the majority of fluids, in which heat conduction must be more influential than some of the effects included by these authors-but to have taken account of heat conduction would have brought on staggering complications in an exact treatment.

B4. Combined hydrodynamics and relaxation (mixture theory). The SakadiMeixner theory, which in suggestion goes back to Einstein, recognizes that for some fluids, at least, "relaxation" exists as a separable phenomenon, while viscous and thermal dissipation exist independently. The two mechanisms are then supposed to operate simultaneously. Since each is non-linear, the result in general is far different from superposing the two effects-this latter being the outcome of Frenkel \& Obrastzov's theory. In absorption, for example, when the frequencies for relaxation resonance and for visco-thermal resonance are far separated, simple superposition can be expected, but when the two frequencies are nearly equal, the mixture theory will yield a complicated and as yet undetermined result.

The Sakadi-Meixner approach has most of the benefits of the visco-elastic method and none of its defects. For it offers the minimum of experimental constants to be fitted, and the mechanical responses which it embodies are responses already strongly indicated by the results of experiment. It is free of the embarrassing variety of mechanical possibilities which is the price Frenkel \& Obrastzov's theory must pay in order to yield a simple superposition formula as its result.

I shall now give a simple physical plausibility argument for the SakadiMeixner theory. Suppose the historical order had been inverted and Greenspan's experiments had been done before those of the 1930's. We should then have begun with a firm confidence in the Navier-Stokes equations, and we should have taken care that all subsequent experiments should have covered a range of values of $S$ sufficient to bring out the shape of the visco-thermal absorption and dispersion curves. For various polyatomic gases and liquids we should then have noticed small irregularities for small values of $S$. These we should have regarded as disturbing departures from the classical theory. From a strictly classical 
molecular view, they would be inexplicable: internal degrees of freedom, for example, would be excited smoothly, giving rise to bulk viscosity but not to any qualitative change in formalism. But with the quantum theory, the internal energy states become discrete, and in circumstances when they are excited the fluid becomes, as far as its gross properties are concerned, a mixture of two or more components. If we are interested only in mechanical problems, we need go no further into quantum theory, but may again apply classical hydrodynamics; this time, however, the hydrodynamics of mixtures, not of pure fluids. This is but reinterpretation of the original view of Kneser-with the important difference that now we see "relaxation" as a quantum mechanical perturbation, in most cases relatively small, in an otherwise classical phenomenon, not as itself the primary occurrence. But it is essential in all cases to use the exact solutions of the appropriate frequency equation.

Recall that for certain fluids, such as carbon disulfide, the simple relaxation theory does not appear to be adequate. While some authors have suggested the superposition of several different relaxation processes, it is at least equally promising to explore the possibility that the internal and visco-thermal resonant frequencies are close enough together that superposition is not valid, and that again it is an exact solution from the two fluid theory which is needed.

This much agreed, there now arises a new mechanical problem. Is each component to be assigned its own viscosity and thermal conductivity, or are these dissipative effects to be taken as properties of the mixture only? ${ }^{7}$ The kinetic theory of gases suggests the latter view, which is in any case the simpler and the easier to explore; this is the model of Sakadi and Meixner.

It remains to test the Sakadi-Meixner theory by experiment, after having obtained the appropriate exact solutions. In this theory, for the case of a two component mixture, there remain essentially four arbitrary parameters: the relaxation constants $K$ and $k$, the ratio of ordinary specific heats $\gamma$, and the thermoviscous number $Y$. From the value of $Y$, the viscosity number $V$ can be determined. There remain then two mechanical questions to be settled by experiment.

1. Is it possible, for any given pure fluid, by adjusting the three parameters $K, k$, and $Y$, to get agreement with experiments covering absorption and dispersion for the full range of values of $X$ ?

2. If the answer to the foregoing is yes, then is $v=\frac{4}{3}$ for all fluids?

The answer to the second question will clarify the nature of bulk viscosity. For if $v=\frac{4}{3}$, then no bulk viscosity is required for the purely visco-thermal dissipation. According to this view, which seems to be held by Meixner, bulk viscosity could then be introduced as a low frequency equivalent of a relaxation process, but on the adoption of a more accurate fluid model all need for it would disappear. Similarly, if in a polyatomic gas bulk viscosity arises solely from internal energy transfer, and if the internal energy levels occur only discretely, then shear viscosity and heat conduction alone should suffice for the remaining dissipation, and we should expect $V=\frac{4}{3}$ in the two fluid model. If, however, even

${ }^{7}$ Cf. $\$ 32$ of the former of my papers cited in footnote 12 of $\$ 4$. 
after adjusting $k$ and $K$ it still remains necessary to give values other than $\frac{4}{3}$ to $\vartheta$, then bulk viscosity independent of relaxation processes will be indicated.

In any case, the experimental existence of both relaxation absorption and dispersion and visco-thermal absorption and dispersion makes the true nature of bulk viscosity difficult to ascertain. From the basic definition (2.6) it is clear that the usual concept of bulk viscosity is formulated within the theory of a pure fluid. If a specific chemically pure fluid, such as carbon dioxide, when subjected to ultrasonic oscillation must be represented by a two fluid model, what value is to be given to its bulk viscosity in other situations, such as a high speed flow past an obstacle? Such a question cannot be settled by ultrasonic measurement or theory. An important example is furnished by Liebermann's experiment, which infers the value of the bulk viscosity from the stream velocity produced by ultrasonic excitation. The formula of Eckart on which the interpretation of the experiment rests is itself a consequence of the Navier-Stokes equations. If the first order terms in these equations are not sufficient to describe the absorption and dispersion of the waves themselves, are we justified in using the second order terms to calculate the stream field accompanying the waves? If a two fluid (relaxation) model is required for absorption and dispersion, should it not be required also to interpret Liebermann's experiment? ${ }^{8}$ That Liebermann's values of $\vartheta$ agree roughly with those determined by "excess" absorption would seem to suggest that it is again some property of the two fluid model which Liebermann has measured, rather than the bulk viscosity of a pure fluid.

It seems that at present we are as far as ever from a clear and consistent method of measuring bulk viscosity. The ultrasonic problem has brought out important gaps in our general understanding of fluid mechanics.

6. The results in this memoir. Unfortunately, the complication of the hydrodynamical mixture theory makes a manageable exact solution difficult. I have had to be content here with the single fluid case (visco-thermal theory), in the hopes that its complete and correct treatment might cast light on what was to be expected and what would have to be done in the more important two fluid case.

Thus the present memoir simply derives, interprets, and tabulates the exact solutions of the Kirchhoff-Langevin equation (4.16). Mathematically, it contains nothing more than the solution of a biquadratic equation. As a contribution to rational mechanics, however, it adds a little to what is known about the NavierStokes equations.

I began by attempting to fit all existing absorption and dispersion data in pure fluids by the results of the simple hydrodynamical theory of this memoir. In $\$ 9$ it is shown why any such attempt must fail. Those more fortunately en-

\footnotetext{
${ }^{8}$ Note that it is not the theoretical existence of the effects which we are questioningboth indeed are predicted by the NAvier-Stokes equations-but that hydrodynamics should be considered quantitatively correct for acoustical streaming while not so for acoustical waves.
} 
dowed with physical intuition have confidently asserted, without precise knowledge of the hydrodynamical solution, that it was insufficient. They are shown to be right by the facts, which have cost some labor to evolve.

Finally, the numerical values tabulated in $\$ 21$ should be useful in exploration of the two fluid theory. In a polyatomic gas for which the relaxation constants have already been evaluated by low frequency measurements, the relaxation frequency (or frequencies) can be compared with that yielding maximum visco-thermal absorption, obtainable from the tables below. If these are far distant from one another, it may be justified in first approximation to superpose the relaxation curves upon the visco-thermal curves. (Such seems to be the case, for example, for ethyl acetate and for carbon dioxide.) If, however, these frequencies are nearly equal, then such superposition will certainly not be valid according to the hydrodynamical mixture theory, and a proper solution of the exact frequency equation of that theory should be obtained. Here is a crucial test of the theory, for according to the visco-elastic proposal of Frenkel \& Obrastzov the two effects may be superposed at all frequencies.

We shall not allude further to these physical questions. The remainder of this memoir is a treatise on the hydrodynamical theory of plane infinitesimal waves in pure fluids. No gain can be had by interrupting the mathematical work with physical cavils. So as to make the results easier to grasp, I shall usually write henceforth as if for any given $\gamma \geqq 1$ and $Y \geqq 0$ a physical fluid $\langle\gamma, Y\rangle$, exactly satisfying the linearized Navier-Stokes at all frequency numbers $X$, exists.

7. Derivation of the characteristic equation of Kirchhoff and Langevin. The linearized equations of continuity, motion, and energy are

$$
\frac{\partial v}{\partial t}-v \frac{\partial u}{\partial x}=0, \quad \frac{\partial u}{\partial t}+v \frac{\partial p}{\partial x}-v(\lambda+2 \mu) \frac{\partial^{2} u}{\partial x^{2}}=0, \quad \theta \frac{\partial \eta}{\partial t}-v \kappa \frac{\partial^{2} \theta}{\partial x^{2}}=0 .
$$

Now for a homogeneous tri-variate fluid we have

$$
\begin{aligned}
\theta \frac{\partial \eta}{\partial t} & =\theta\left[\left(\frac{\partial \eta}{\partial v}\right)_{\theta} \frac{\partial v}{\partial t}+\left(\frac{\partial \eta}{\partial \theta}\right)_{v} \frac{\partial \theta}{\partial t}\right]=\theta\left(\frac{\partial p}{\partial \theta}\right)_{v} \frac{\partial v}{\partial t}+c_{v} \frac{\partial \theta}{\partial t} \\
& =\frac{c_{p}-c_{v}}{\left(\frac{\partial v}{\partial \theta}\right)_{p}} \frac{\partial v}{\partial t}+c_{v} \frac{\partial \theta}{\partial t}
\end{aligned}
$$

where the last two steps follow by well known thermodynamical identities. ${ }^{1}$ Hence $(7.1)_{3}$ becomes

$$
\frac{\partial \theta}{\partial t}+\frac{\theta(\gamma-1)}{v} \mathfrak{F} \frac{\partial v}{\partial t}-\frac{v \kappa}{c_{v}} \frac{\partial^{2} \theta}{\partial x^{2}}=0
$$

${ }^{1}$ See $e . g . \$ 25$ of P. S. EPsTein, Textbook of thermodynamics, N. Y. (1937). 
where

$$
\mathfrak{F} \equiv \frac{v}{\theta\left(\frac{\partial v}{\partial \theta}\right)_{p}}=\frac{1}{\theta \alpha_{\theta}}
$$

For a piezotropic fluid a result of the same form but with $\mathfrak{F}=0$ is evidently valid.

Now write

$$
v=v_{0}(1+\Upsilon), \quad \theta=\theta_{0}(1+\theta)
$$

and suppose $\Upsilon \ll 1, \Theta \ll 1$. For a homogeneous fluid we have then

$$
p=p_{0}[1-\mathfrak{S} \Upsilon+\mathfrak{S} \theta]
$$

where

$$
\mathfrak{S} \equiv-\frac{v}{p}\left(\frac{\partial p}{\partial v}\right)_{\theta}, \quad \mathfrak{S} \equiv \frac{\theta}{p}\left(\frac{\partial p}{\partial \theta}\right)_{v} .
$$

For a piezotropic fluid we have from (7.7) and (2.7)

$$
\mathfrak{S}=0, \quad \mathfrak{S}=\frac{V_{0}^{2}}{p v},
$$

while for a tri-variate fluid we have identically

$$
\text { (B) = FS. }
$$

In the special case of a perfect gas, $\mathfrak{F}=\mathfrak{S}=\mathfrak{S}=1$. We notice also that for a tri-variate fluid we have in contrast to $(7.8)_{2}$

$$
\begin{aligned}
\gamma \frac{p v \Theta}{V_{0}^{2}} & =\frac{\left(\frac{\partial \eta}{\partial \theta}\right)_{p}\left(\frac{\partial p}{\partial v}\right)_{\theta}}{\left(\frac{\partial \eta}{\partial \theta}\right)_{v}\left(\frac{\partial p}{\partial v}\right)_{\eta}}=\frac{\frac{\partial(\eta, p)}{\partial(\theta, p)} \frac{\partial(\theta, p)}{\partial(\theta, v)}}{\left(\frac{\partial \eta}{\partial \theta}\right)_{v}\left(\frac{\partial p}{\partial v}\right)_{\eta}} \\
& =\frac{\frac{\partial(\eta, p)}{\partial(\theta, v)}}{\left(\frac{\partial \eta}{\partial \theta}\right)_{v}\left(\frac{\partial p}{\partial v}\right)_{\eta}}=\frac{\frac{\partial(\eta, v)}{\partial(\theta, v)} \frac{\partial(\eta, p)}{\partial(\eta, v)}}{\left(\frac{\partial \eta}{\partial \theta}\right)_{v}\left(\frac{\partial p}{\partial v}\right)_{\eta}}=1
\end{aligned}
$$

(In words, the ratio of the squares of the isentropic and isothermal speeds of sound is $\gamma$, for any tri-variate fluid.)

Putting (7.5) and (7.6) into the system $(7.1)_{1},(7.1)_{2}$, and (7.3) yields 


$$
\begin{gathered}
\frac{\partial \Upsilon}{\partial t}-\frac{\partial u}{\partial x}=0 \\
-p v \mathfrak{S} \frac{\partial \Theta}{\partial x}+p v \circlearrowleft \frac{\partial \Upsilon}{\partial x}-\frac{\partial u}{\partial t}+v(\lambda+2 \mu) \frac{\partial^{2} u}{\partial x^{2}}=0 \\
\frac{\partial \Theta}{\partial t}-\frac{v \kappa}{c_{v}} \frac{\partial^{2} \Theta}{\partial x^{2}}+(\gamma-1) \mathfrak{F} \frac{\partial \Upsilon}{\partial t}=0
\end{gathered}
$$

where all coefficients are to be evaluated at the condition $v=v_{0}, \theta=\theta_{0}$, subscript noughts having been omitted in the writing out. ${ }^{2}$

The linear system (7.11) possesses solutions of the type (1.2) if and only if $\sigma$ satisfy the characteristic equation

$$
0=\left|\begin{array}{ccc}
0 & i \omega & -\frac{\sigma \omega}{2 \pi V_{0}} \\
-\frac{p v \mathfrak{S} \sigma \omega}{2 \pi V_{0}} & \frac{p v \oiint \sigma \omega}{2 \pi V_{0}} & -i \omega+\frac{v(\lambda+2 \mu) \sigma^{2} \omega^{2}}{4 \pi^{2} V_{0}^{2}} \\
i \omega-\frac{v \kappa \sigma^{2} \omega^{2}}{4 c_{v} \pi^{2} V_{0}^{2}} & (\gamma-1) \Im i \omega & 0
\end{array}\right|
$$

which is easily put into the form

$$
\begin{aligned}
\left(-i+X \frac{\sigma^{2}}{4 \pi^{2}}\right)(i- & \left.\gamma X Y \frac{\sigma^{2}}{4 \pi^{2}}\right) \\
& -\frac{i \sigma^{2} p v \mathscr{S}}{4 \pi^{2} V_{0}^{2}}\left[\left\{(\gamma-1) \frac{\mathfrak{F S}}{\mathfrak{S}}+1\right\} i-\gamma X Y \frac{\sigma^{2}}{4 \pi^{2}}\right]=0 .
\end{aligned}
$$

For piezotropic fluids, as already remarked, we have $\gamma=1, \mathfrak{F}=\mathfrak{S}=0$, whence and by $(7.8)_{2}$ we see that (7.13) factors at once:

$$
\left[-i+(X-i) \frac{\sigma^{2}}{4 \pi^{2}}\right]\left[i-X Y \frac{\sigma^{2}}{4 \pi^{2}}\right]=0 .
$$

The former factor pertains to pressure waves, the latter to thermal waves.

For tri-variate fluids we use the identities (7.9) and (7.10), by whose aid (7.13) reduces to the Kirchhoff-Langevin equation (4.16).

8. Further remarks on the piezotropic case. From (7.14) we obtain two resolutions of the type (1.13): $G=1, H=X$ for pressure waves, while $G=0, H=X Y$ for thermal waves. By applying (1.15) we quickly derive (4.10) and (4.23). In $\$ 4$ the direct consequences of these formulae have been obtained and discussed. Here we shall pause to consider their physical relevance.

2 To include radiation according to NewTon's law of cooling (see the discussion of STOKEs's work in $\$ 4$ ), add $q \Theta$ to the left hand side of $(7.11)_{3}$. 
Despite the fact that it is theoretically possible in a piezotropic fluid to obtain a solution representing pressure waves unaccompanied by thermal waves or conversely, nevertheless such a separation would be most difficult to effect in an experiment, and the remarks of Kirchhoff (\$4) remain physically appropriate in this case, too. A typical source of disturbance, such as the face of a crystal, when set into very rapid oscillation may be regarded in first approximation as moving isentropically. In consequence it will suffer rapid oscillations of temperature, which will be propagated into the fluid medium along with the pressure pulses. A receiving instrument will accept waves of both

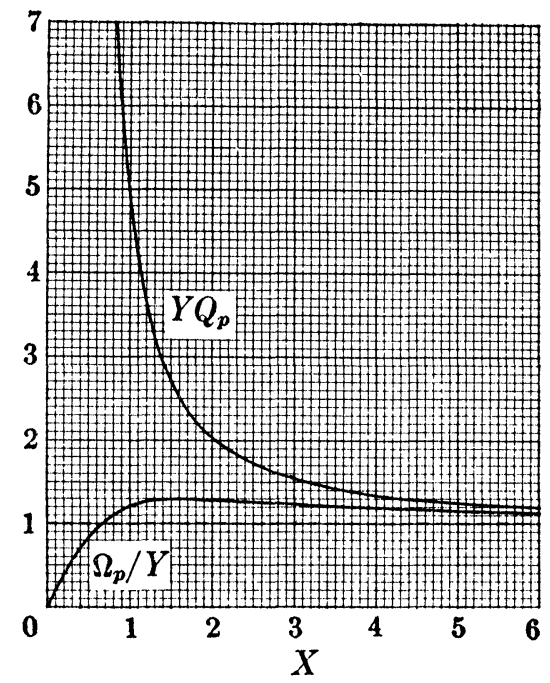

Fig. 8.1

Relative measures for a piezotropic fluid

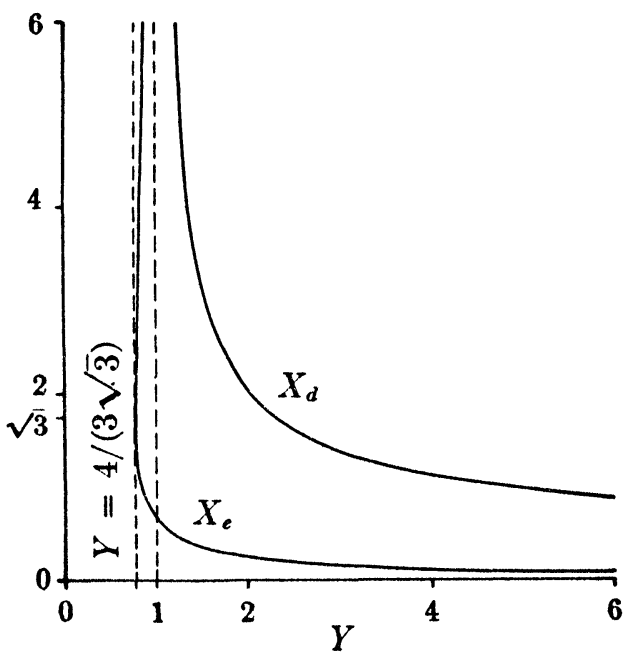

FIG. 8.2

Dividing and overtaking frequency numbers, $X_{d}$ and $X_{e}$, for a piezotropic fluid

types and be affected by both; indeed, when a rapid thermal oscillation falls upon a receiver, that receiver will also tend to be set into isentropic motion, and the consequent alternations of shape and volume cannot of themselves be distinguished from those excited by pressure waves. It is of moment, therefore, to study both types of waves.

For the relative measures (1.16) and (1.17) we have

$$
\begin{gathered}
\frac{\Omega_{p}}{Y}=\frac{X\left(1+\sqrt{1+X^{2}}\right)}{1+X^{2}} \\
Y Q_{p}=\frac{1+X^{2}}{X\left(\sqrt{1+X^{2}}-1\right)}=\left(1+\frac{1}{X^{2}}\right) \frac{1+\sqrt{1+X^{2}}}{X}
\end{gathered}
$$

Thus it is possible to draw universal curves of the relative measures (Fig. 8.1), valid for all piezotropic fluids, the effect of different values of heat conductivity 
appearing only through a change of scale. This change of scale, however, enables us to obtain strikingly different results for different values of $Y$.

The quantity $\Omega_{p} / Y$ increases rapidly with $X$, reaching the value 1 when $X$ satisfies $1-2 X+2 X^{2}-2 X^{3}=0$, viz. at $X=0.6478 \cdots$. Its maximum value, $3 \sqrt{ } \overline{3} / 4$, occurs when $X=\sqrt{3}$, the same frequency number which yields the maximum for the coefficient $A_{0 p}$ for pressure waves. Thereafter $\Omega_{p} / Y$ steadily approaches 1 from above as $X \rightarrow \infty$.

The function $Y Q_{p}$ decreases steadily from the value $\infty$ when $X=0$ to the value 1 when $X=\infty$. Thus if $Y<1$ we have $Q>1$ at all frequencies. If $Y>1$, however, there exists a dividing frequency number $X_{d}$ below which $Q>1$, above which $Q<1$. This frequency number is the single positive root of the equation

$$
1+2 Y X+2 X^{2}+2 Y X^{3}-\left(Y^{2}-1\right) X^{4}=0 .
$$

To find an overtaking frequency number $X_{e}$ at which the two types of waves travel at the same speed, we set $\Omega_{p}=1$, obtaining

$$
1-2 Y X+2 X^{2}-2 Y X^{3}-\left(Y^{2}-1\right) X^{4}=0 .
$$

When $Y<4 /(3 \sqrt{3})$ there is no positive root. When $Y=4 /(3 \sqrt{3})$ there is the single positive root $X=\sqrt{3}$. When $4 /(3 \sqrt{3})<Y<1$ there are two positive roots, one of which exceeds $\sqrt{3}$, while the other lies between $\sqrt{3}$ and $0.6478 \cdots$. When $Y \geqq 1$ there is again a single root, and as $Y$ varies from 1 to $\infty$ this root steadily falls off from $0.6478 \cdots$ to zero.

The curves of $X_{d}$ and $X_{e}$ as functions of $Y$ are shown in Fig. 8.2.

Our conclusions are summarized in the following

Relative measures theorem for piezotropic fluids. a. At low frequencies the thermal waves are absorbed enormously more than are pressure waves, while travelling much more slowly. As frequency increases, this situation tends to reverse itself. In particular, the relative absorption $Q$ is a monotone decreasing function of $X$.

b. For all values of $Y$

$$
\Omega_{p} \rightarrow Y, \quad Q_{p} \rightarrow \frac{1}{\bar{Y}} \quad \text { as } \quad X \rightarrow \infty .
$$

c. The greatest speed of thermal waves relative to pressure waves is $3 \sqrt{3} Y / 4$, occurring when $X=\sqrt{3}$. When $X<\sqrt{3}, \Omega_{p}$ increases; when $X>\sqrt{3}, \Omega_{p}$ decreases.

d. If $Y<1$, pressure waves are never at any frequency absorbed so strongly as are thermal waves. If $Y<4 /(3 \sqrt{3})$, pressure waves travel faster than thermal waves at all frequencies.

e. If $4 /(3 \sqrt{3}) \leqq Y<1$, there are two positive roots $X_{e 1}$ and $X_{e 2}$ of (8.4). When $Y=4 /(3 \sqrt{3})$, these two coincide. At these overtaking frequency numbers $X_{e 1}$ and $X_{e 2}$, then, pressure waves and thermal waves travel with equal speed; when $X<X_{\text {e1 }}$ the pressure waves are speedier; when $X_{e 1}<X<X_{e 2}$ the thermal waves are speedier; and when $X>X_{e 2}$ it is again the pressure waves which travel faster.

f. If $Y \geqq 1$, there is a dividing frequency number $X_{d}$, the single positive root of 
(8.3), below which thermal waves are more strongly absorbed, but above which pressure waves are more strongly absorbed (when $Y=1, X_{d}=\infty$ ). There is then but a single positive root $X_{e}$ of (8.4); when $X>X_{e}$ thermal waves are more speedy than pressure waves; conversely when $X<X_{e}$. As $Y$ increases from 1 to $\infty, X_{e}$ decreases from $0.6478 \cdots$ to 0 , while $X_{d}$ decreases from $\infty$ to 0 .

These results foreshadow the distinction for tri-variate fluids between the two cases $Y<1, Y>1$, a distinction which will later appear to be essential $(\$ \$ 12-13)$.

9. The general case: the two types of waves and the three major types of fluids. We write the Kirchhoff-Langevin frequency equation (4.16) in the form:

$$
\left(\frac{2 \pi}{\sigma}\right)^{4}+\left(\frac{2 \pi}{\sigma}\right)^{2}[1+i X(1+\gamma Y)]+X Y[-\gamma X+i]=0 .
$$

Hence

$$
\begin{aligned}
& -2\left(\frac{2 \pi}{\sigma}\right)^{2} \\
& \quad=1+i X(1+\gamma Y)+\left[1-X^{2}(1-\gamma Y)^{2}+2 i X(1-[2-\gamma] Y)\right]^{\frac{1}{2}},
\end{aligned}
$$

one pair of roots $\sigma$ corresponding to each determination of the square root.

If our task were merely to find the numerical values of the two pairs of roots $\sigma$ for a given fluid $\langle\gamma, Y\rangle$ at given frequency number $X$, it would be a trivial matter. Such is not the case, however. First, each pair of roots yields different values for the absorption and dispersion measures, and we must know which ones should be compared with measurements. Second, we shall wish to discuss the behavior of the various measures as the frequency number $X$ and also, in so far as is possible, the fluid $\langle\gamma, Y\rangle$ is permitted to vary. Thus there must be some connection between the determinations at different values of the variables.

The problem may be approached through either of these two avenues. We choose the second, and proceed at the beginning in a purely formal way, deferring the former and more important question of the physical meaning of the results until more knowledge about the solutions themselves has been accumulated.

Suppose $\gamma Y \neq 1$ and write

$$
\begin{gathered}
\Theta \equiv X|1-\gamma Y|, \quad K \equiv \frac{1+\gamma Y}{|1-\gamma Y|}, \\
L \equiv \frac{1-(2-\gamma) Y}{|1-\gamma Y|}, \quad z \equiv 1-Q^{2}+2 i L Q .
\end{gathered}
$$

With these notations $(9.2)$ becomes

$$
-2\left(\frac{2 \pi}{\sigma}\right)^{2}=1+i K \Theta+z^{\frac{1}{2}}
$$


For a particular fluid $\langle\gamma, Y\rangle$, as $X$ varies from 0 to $+\infty$ the variable $z$ describes in the $z$-plane the half parabola

$$
x=1-Q^{2}, \quad y=2 L Q,
$$

whose imaginary intercept is $2 L$. If $L>0$, this curve lies in the upper half plane; if $L=0$, it degenerates into the real axis from +1 to $-\infty$; if $L<0$, it lies in the lower half plane. Now for the piezotropic fluids $\langle 1, Y\rangle$ we have $L=1$ provided $Y<1$, and also $L=1$ for non-conductors $\langle\gamma, 0\rangle$. For $\gamma$ and $Y$ slightly above 1 and 0 we have $L \geqq 1$, and this fact suggests subdivision of the case $L \geqq 0$ into three more: (a) $0 \leqq \gamma Y<1$, so that $\infty>L \geqq 1$; (b) $\gamma^{-1}<$ $Y \leqq 1$, whence again $\infty>L \geqq 1$; (c) $\gamma \geqq 2$, or $1<Y<(2-\gamma)^{-1}$, so that $0<L<1$. One notes that in the cases (a) and (b) the totalities of the parabolas coincide, but that none of those in case (c) coincide with any in cases (a) and (b) or in the cases $L=0$ or $L<0$. One notes also that the parabola for the fluids $\langle\gamma, 1\rangle$ with $\gamma \neq 1$, which are included in (b), coincides with that for piezotropic fluids $\langle 1, Y\rangle$ with $Y<1$, which are included in case (a). Finally, from (9.2) we see that in the case $\gamma Y=1, \gamma \neq Y, z$ traverses a half line extending vertically upward from the point $z=+1$.

To obtain solutions of (9.4) which are continuous functions of $\gamma, Y$, and $X$, it is sufficient to cut the $z$-plane along the negative real axis. The results of the paragraph preceding show that in so doing we exclude those fluids for which $L=0$, and we make it impossible to pass from fluids for which $L>0$ to those for which $L<0$. This fact, taken together with the statements of the paragraph preceding, suggests the classification of fluids which follows.

\section{Nomenclature for fluids}

\section{Defining relation}

The three major types:

$$
\begin{aligned}
& 0 \leqq Y<\gamma^{-1} \\
& Y>\gamma^{-1}, \quad(2-\gamma) Y<1 \\
& \gamma<2, \quad(2-\gamma)^{-1}<Y<\infty
\end{aligned}
$$

Range of values

Subtypes of moderate conductors:

$$
\begin{array}{ll}
\gamma^{-1}<Y \leqq 1 & 1 \leqq L<\infty \\
\gamma \geqq 2 \text { or } 1<Y<(2-\gamma)^{-1} & 0<L<1
\end{array}
$$

Singular and special cases:

$$
\begin{array}{ll}
\gamma \neq 1, \quad(2-\gamma)^{-1}=Y & L=0 \\
\gamma \neq 1, \quad \gamma Y=1 & L=\infty
\end{array}
$$
for $L$

Name of fluid class

$\begin{array}{ll}1 \leqq L<\infty & \text { weak conductors } \\ 0<L<\infty & \text { moderate conductors } \\ -1 \leqq L<0 & \text { strong conductors }\end{array}$

$1 \leqq L<\infty \quad$ submoderate conductors (subcase of moderate conductors) supermoderate conductors (subcase of moderate conductors)

singular fluids A (boundary between moderate and strong conductors) singular fluids B (boundary between weak and moderate conductors) 


$$
\begin{array}{lll}
Y=0 & L=1 & \begin{array}{l}
\text { non-conductors } \\
\text { (special case of weak conductors) } \\
\text { piezotropic fluids } \\
\text { (if } Y<1, \text { special case of weak } \\
\text { conductors }(L=+1) ; \text { if } Y>1, \\
\text { special case of strong conduc- } \\
\text { tors }(L=-1) \text { ) }
\end{array} \\
Y=1, \gamma \neq 1 & L=1 & \begin{array}{l}
\text { pseudopiezic fluids } \\
\text { (boundary between submoderate } \\
\text { and supermoderate conductors; } \\
\text { special case of the former) }
\end{array}
\end{array}
$$

The foregoing classification of fluids is indicated diagrammatically in Figs. 9.1 and 9.2. The former shows the several regions of the $\gamma-Y$ plane, while the latter exhibits the appropriate argument curves in the $z$-plane.

From these drawings, as from the preceding analysis, emerges an indication of the highest importance: piezotropic fluids furnish a limiting case either for weak conductors or for strong conductors, according as $Y<1$ or $Y>1$. In $\$ 18$ we shall see from a special case of a duality theorem that piezotropic fluids are typical of moderate conductors also. In fact, a major concern of the whole investigation is to show that piezotropic fluids $\langle 1, Y\rangle$, if properly used, can yield at least approximate information about the absorption and dispersion measures in nearly all fluids $\langle\gamma, Y\rangle$.

In Fig. 9.3 a number of physical fluids are represented in a $\gamma-Y$ diagram. Recalling that the value of $V$ is not well known, but that $V \geqq \frac{4}{3}$, we put dots for $3 /(4 P)$ instead of $Y$, adding an arrow to indicate that the value of $Y$ may lie anywhere below the dot on the same vertical. A small cross indicates the value $Y$ resulting from Liebermann's experimental determination ${ }^{1}$ of $\lambda / \mu$ by the method of acoustical streaming, when such a value exists. A small circle indicates that the exact theoretical measures for the corresponding fluid are tabulated in $\$ 21$.

We have said already that for any fluid $\langle\gamma, Y\rangle$ not one of the singular fluids $A$ the argument $z$ of the square root in (9.2) varies with $X$ in a proper cut plane. This fact legitimizes the following

Principal definition: Waves corresponding to the two values of $\sigma$ given by the principal determination of the square root, $\sqrt{ } \bar{z}$, in (9.2), will be called type I waves; waves corresponding to the two values given by the other determination, $-\sqrt{ } z$, will be called type II waves.

The principal definition fails to apply to the singular fluids A. For convenience we shall postpone discussion of both types of singular fluid until $\$ 20$, understanding that except in case of specific mention to the contrary, no statement in the sequel is to be taken as applying to either type of singular fluid.

Now since for all fluids both the first term and the argument of the square root in (9.2) are polynomials in $\gamma, Y$, and $X$, each determination of $\sigma^{2}$ will be an

${ }^{1}$ Op. cit. $\$ 4$, Note 70. 
analytic function of each of these same variables, so long as $\langle\gamma, Y\rangle$ be kept from crossing the cut $L=0$. Hence we obtain the

Lemma. Let the frequency number $X$ vary continuously in any way, and simultaneously let the fluid $\langle\gamma, Y\rangle$ vary continuously either over the range of strong

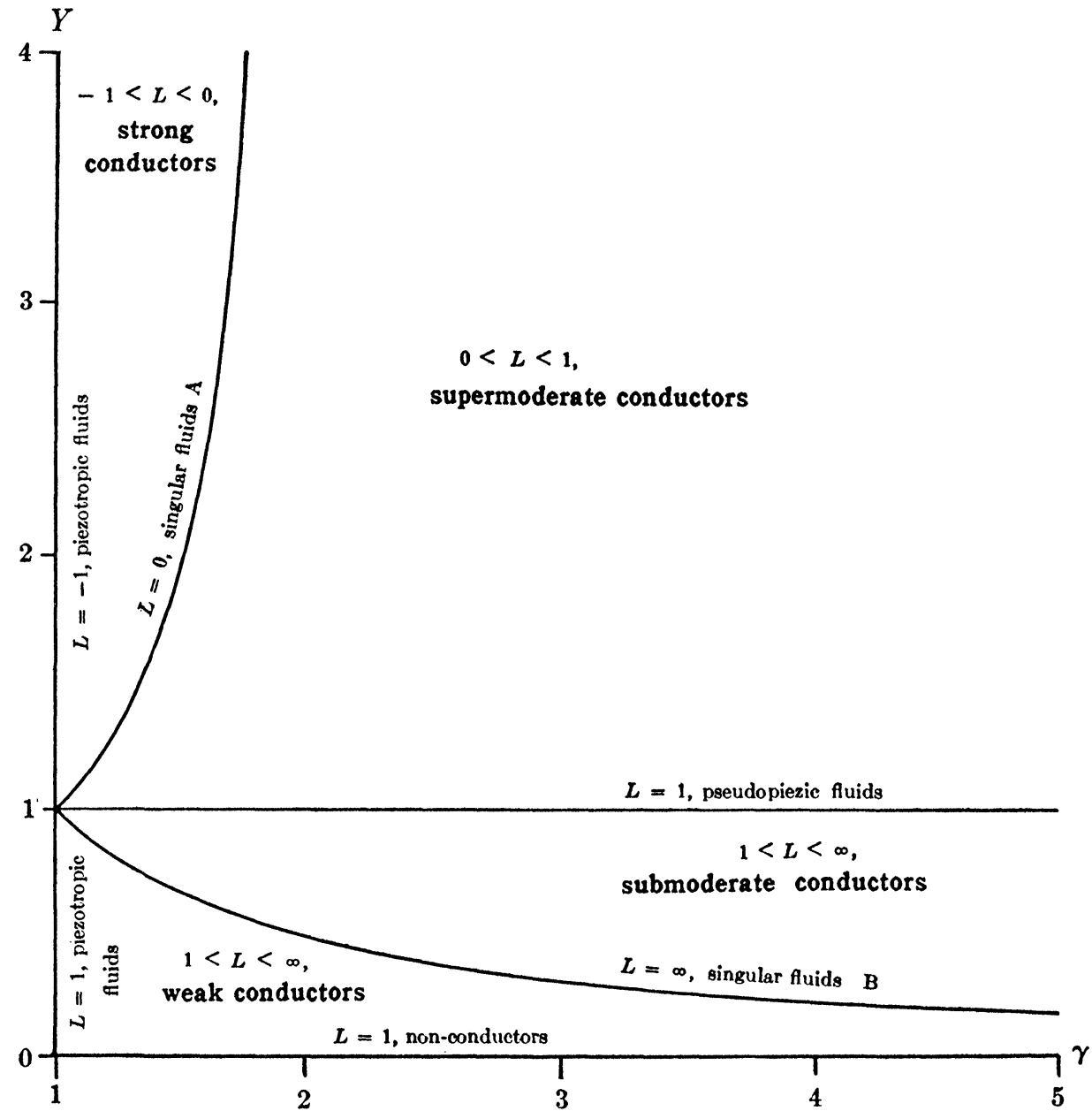

FIG. 9.1

Classification of fluids in the $\gamma-Y$ plane

conductors or over the range of weak conductors, singular fluids $B$, and moderate conductors. Then each determination of $\sigma^{2}$ is an analytic function of $X, \gamma$, and $Y$.

Now (1.15) expresses the absorption and dispersion measures in terms of the coefficients $G$ and $H$ in (1.13), regardless of which root $\pm \sigma$ is selected. The lemma 
asserts that $G$ and $H$ vary analytically with $X, \gamma$, and $Y$, subject to stated restrictions. To show that the several measures are analytic also, it is then sufficient to make sure that $G^{2}+H^{2} \neq 0$, or, equivalently, $\sigma^{-2} \neq 0$, in the regions considered.

For all fluids except the singular fluids B, (9.4) and (9.5) yield $\sigma^{-2}=0$ only

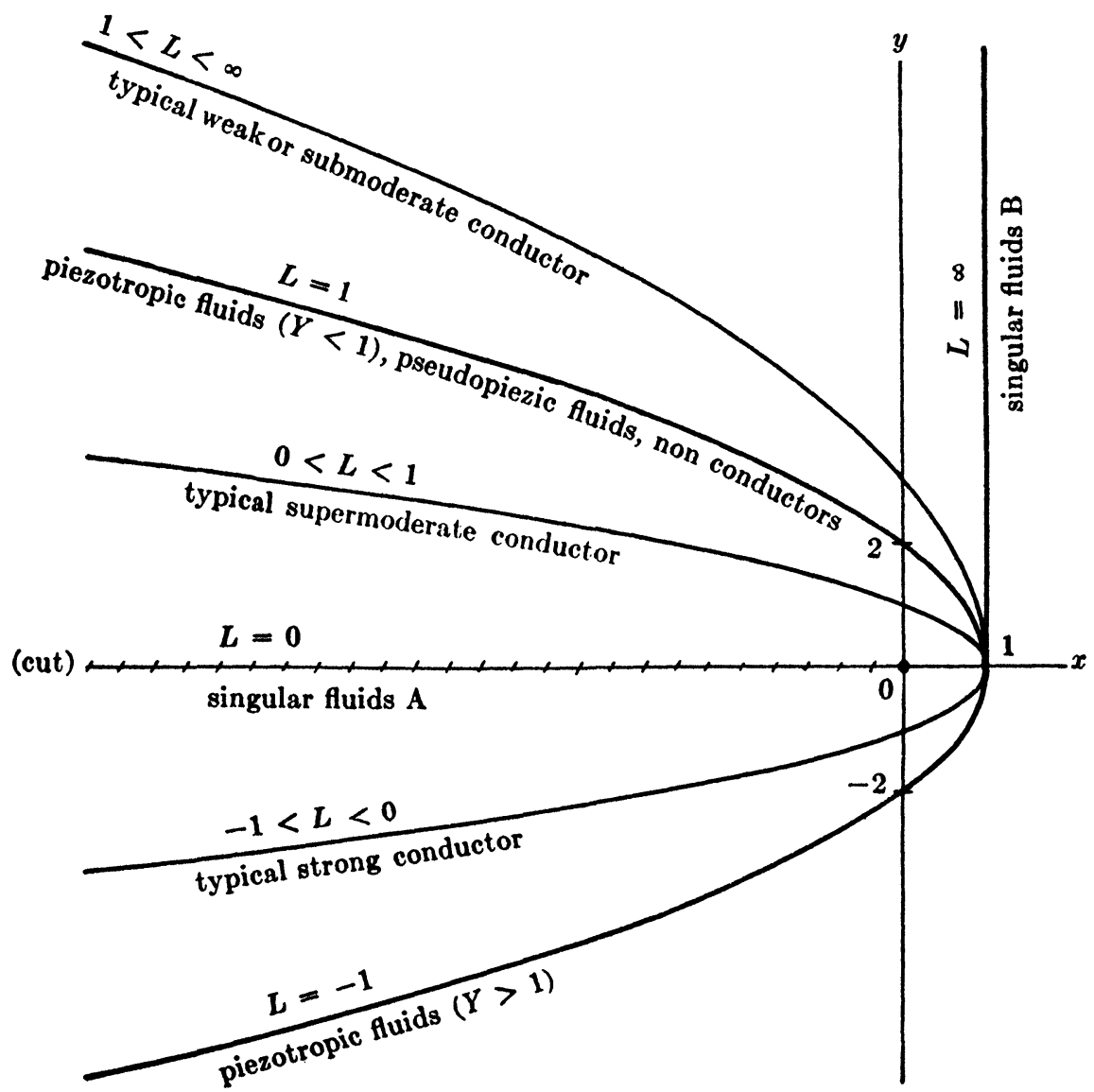

Fig. 9.2

Argument curves in the $z$-plane for the various fluid types

if $Q=0$ or $K=L=1$; the former condition implies $X=0$, while the latter condition implies $Y=0$, and both then yield $\sigma^{-2}=0$ for type II waves only. For the singular fluids $\mathrm{B}, \sigma^{-2}=0$ only if $X=0$, and then only for type II waves. In summary of these remarks, we have the

Analyticity theorem. All absorption and dispersion measures for type $I$ waves are analytic functions of $X, \gamma$, and $Y$, provided only that $\langle\gamma, Y\rangle$ remain either in 


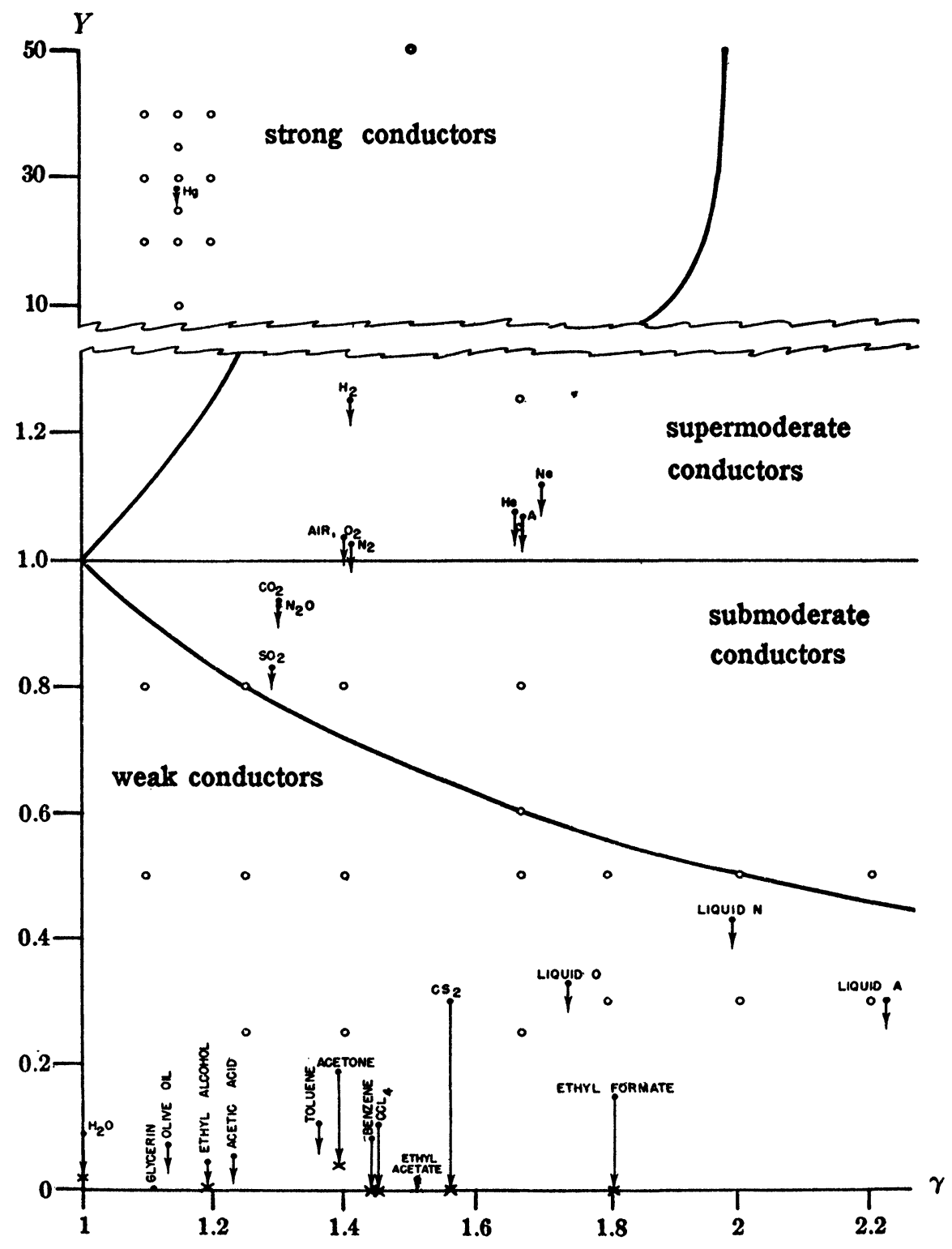

Fig. 9.3

Physical fluids in the $\gamma-Y$ plane

A dot indicates the location of the fluid if the Stokes relation holds. The arrows indicate that positive bulk viscosity has the effect of depressing the fluid toward the non-conductor line $Y=0$. The crosses indicate the location of the fluid if Liebermann's values for bulk viscosity are employed. The circles indicate fluids $\langle\gamma, Y\rangle$ for which the exact solutions are tabulated in $\$ 21$. 
the range of strong conductors or in the range of weak and moderate conductors and singular fluids B. If $X Y>0$, the same holds for type II waves also.

This theorem indicates that our formal separation of waves into two types furnishes a rational basis for further inquiry. Furthermore, it justifies interpolating when using the tables given below-interpolation in frequency for any given fluid, or interpolation between two different fluids, provided that both or neither are strong conductors.

The distinction made between the different fluid types in the statement of the analyticity theorem is essential if we insist on letting $X$ vary over the whole range $0 \leqq X<\infty$. If, however, we add the restriction $\theta<1$, then all the parabolic segments (9.5) lie in the cut plane, and for such variations, consequently, $\sqrt{z}$ will remain analytic. Hence follows the

Analyticity theorem for low frequencies. In the analyticity theorem, suppose only frequencies such that

$$
X<\frac{1}{|1-\gamma Y|}
$$

be considered. Then all restriction on the fluids $\langle\gamma, Y\rangle$ may be removed, and the result of the theorem still holds.

It is this theorem which explains why in the usual treatments, which are valid strictly only in the limit of zero frequency, no separation of fluids into classes has been necessary.

The next step is to consider the relation of the two types of waves to those in piezotropic fluids. First, it is trivial to remark from comparison of (9.2) with (4.10) and (4.23) that when $\gamma=1$ type I waves are pure pressure waves, while type II waves are pure thermal waves. We now consider the limit $\gamma \rightarrow 1+$. Since the inequality $\gamma^{-1}<Y<(2-\gamma)^{-1}$ which defines moderate conductors cannot be satisfied up to and including the value $\gamma=1$ on any path leading to the fluid $\langle 1, Y\rangle$ in the $\gamma-Y$ plane, whatever the value of $Y$, the question of limit behavior as $\gamma \rightarrow 1+$ has no meaning for moderate conductors. For both weak and strong conductors, however, the continuity theorem stated above yields a positive result, expressed in the following

First limit theorem $(\gamma \rightarrow 1)$. Consider a set of points $\left\langle\gamma_{P}, Y_{P}\right\rangle$ lying wholly in either the weak conductor region or the strong conductor region, and having as limit the fluid $\langle 1, Y\rangle$. Then, for any frequency number $X$, as $\left\langle\gamma_{P}, Y_{P}\right\rangle \rightarrow\langle 1, Y\rangle$ the measures for type $I$ waves continuously approach those for pure pressure waves in a piezotropic fluid. If $Y_{P}>0, Y>0, X>0$, the measures for type II waves continuously approach those for pure thermal waves in the piezotropic fluid $\langle 1, Y\rangle$.

This theorem must not be interpreted as implying that type I waves are pure pressure waves in general, since, as was noted in $\S 4$, if $\gamma \neq 1$ pressure waves and temperature waves are inextricably linked together. The physical significances of the two types of waves will be discussed in $\S 13$. The present theorem 
is important, however, in that it again suggests that the piezotropic fluid serves as a first approximation to a weak conductor when $Y<1$, to a strong conductor when $Y>1$, since it is a limiting case for each type.

In a piezotropic fluid the behavior of type I waves is independent of the value of $Y$. Accordingly it is interesting to discuss the limit $Y \rightarrow 0$ in general. As $Y \rightarrow 0$, whatever the value of $\gamma$, the principal determination of the root in (9.2) approaches $1+i X$ and hence we have the following

Second limit theorem $(Y \rightarrow 0)$. In the limit of vanishing thermal conductivity, whatever the value of $\gamma$, the measures for type I waves continuously approach those for pressure waves in a piezotropic fluid.

This theorem enables us to prove rigorously that the visco-thermal theory cannot account for all existing absorption and dispersion data. For suppose, in the case of a fluid such as benzene or carbon dioxide, where the "excess" absorption coefficient at the lowest frequencies for which data is available is tens or hundreds of the "classical" coefficient, we adjust the number $V$ by (4.32) so that at these low frequencies the visco-thermal theory agrees with the data. Since $Y=(P V)^{-1}$, a large value of $V$ results in a very small value of $Y$. The examples given in Table 3.1 show that in a great many cases one gets values of $Y$ such as 0.1 or even less. By the second limit theorem, we are then justified ${ }^{2}$ in regarding these fluids as very nearly piezotropic, as far as their absorption and dispersion are concerned. Now the large values of $v$ imply that a relatively small frequency will yield a fairly large $X=v S$ (see Table 3.1 ); hence the non-linear parts of the absorption and dispersion curves in Fig. 4.1 will correspond to reasonably small frequencies in cycles (i.e., tens of megacycles or even less, at normal conditions). Thus we obtain qualitative agreement. But quantitative agreement is lacking. For by $(4.12)_{2}$ the coefficient $A_{0 p}$ increases until it attains a maximum value $2.22 \cdots$, while the measured absorption peaks in many fluids are not nearly so high. (Numerical cases reveal other quantitative disagreements: with $v$ so adjusted the visco-thermal theory yields linear absorption over too great a frequency range, and a resonant frequency which is too high, besides dispersion which is too small at low frequencies and too large at moderate frequencies. $C f$. the general theorems of $\$ 4 C$.) Thus, definitively, in many fluids the absorption predicted by the visco-thermal theory (with adjusted bulk viscosity) is greater than that observed at the lowest frequencies where non-linear absorption occurs.

It is curious that the final reason for rejecting the visco-thermal theory as the complete theory of ultrasonics should be the direct opposite of that which gave rise to the relaxation theory originally.

But, more important, our result shows that determining bulk viscosity by low frequency absorption data (i.e., by (4.32)) cannot be correct, since in general agree-

\footnotetext{
2 There is an omission in the reasoning here, since the second limit theorem refers to type I waves only. This gap will be filled by the proof at the end of $\$ 11$ that when $Y$ is small type II waves are far more strongly absorbed than are type I waves.
} 
ment between theory and experiment will then not follow beyond the range of linear absorption.

10. General exact expressions for the absorption and dispersion measures. Still proceeding formally with (9.2), put

$$
E \equiv 1-X^{2}(1-\gamma Y)^{2}, \quad F \equiv 2 X(1-[2-\gamma] Y) .
$$

Then in weak and moderate conductors $F>0$, while in strong conductors $F<0$. By applying (1.11) to (9.2), from the definitions of the previous section we obtain the following resolutions of the type (1.13):

$$
\begin{gathered}
2 G=1 \pm \frac{1}{\sqrt{2}} \sqrt{\sqrt{E^{2}+F^{2}}+E} \\
\pm(1+\gamma Y)\left\{\begin{array}{l} 
\pm \frac{1}{\sqrt{2}} \sqrt{\sqrt{E^{2}+F^{2}}-E} \\
\quad \text { for weak and moderate conductors, } \\
\mp \frac{1}{\sqrt{2}} \sqrt{\sqrt{E^{2}+F^{2}}-E} \text { for strong conductors, }
\end{array}\right.
\end{gathered}
$$

where in all cases the upper sign yields type I waves, the lower sign, type II waves.

By substituting (10.2) and (10.3) into (1.15), exact expressions for all absorption and dispersion measures may be obtained. These, however, are so elaborate that their interpretation is difficult. For certain fluids $\langle\gamma, Y\rangle$, numerical values of all measures are tabulated in \$21. The remainder of the analysis obtains simple approximations, as well as some information about the physical significance of the two types of waves.

11. Approximation for very low and moderately low frequencies. The formulae of the previous section enable us by straightforward but laborious calculations to obtain the leading terms in the power series expansions in $X$ of the various absorption and dispersion measures. For type I waves in all fluids the results are:

$$
\begin{aligned}
& r=1+\frac{1}{4} X^{2}\{3+(\gamma-1) Y(10-[7-3 \gamma] Y)\}+O\left(X^{4}\right) \\
& \begin{aligned}
a=\pi X\left[1+(\gamma-1) Y-X^{2}\{1+\right. & (\gamma-1) Y(6+2[3 \gamma-5] Y \\
& \left.\left.\left.+\left[\gamma^{2}-5 \gamma+5\right] Y^{2}\right)\right\}\right]+O\left(X^{5}\right)
\end{aligned} \\
& \begin{aligned}
A=\pi X\left[1+(\gamma-1) Y-\frac{1}{4} X^{2}\{1+\right. & (\gamma-1) Y(11-[23-11 \gamma] Y \\
& \left.\left.\left.+\left[\gamma^{2}-10 \gamma+13\right] Y^{2}\right)\right\}\right]+O\left(X^{5}\right),
\end{aligned}
\end{aligned}
$$




$$
\begin{aligned}
A_{0}=\pi X\left[1+(\gamma-1) Y-\frac{1}{8} X^{2}\{5\right. & +(\gamma-1) Y(35+[35 \gamma-63] Y \\
+ & {\left.\left.\left.\left[5 \gamma^{2}-30 \gamma+33\right] Y^{2}\right)\right\}\right]+O\left(X^{5}\right) . }
\end{aligned}
$$

For any values of $Y$ and $\gamma$, there exists a range of small values of $X$ such that the foregoing formulae are very accurate. A sufficient condition is that $X$ shall be negligible with respect to the following four quantities:

$$
\begin{array}{cc}
\frac{1}{\sqrt{2}|1-\gamma Y|}, & \frac{1}{2|1-(2-\gamma) Y|}, \quad \frac{1}{1+(\gamma-1) Y}, \\
& \frac{1+(\gamma-1) Y}{Y|1-Y||1-(2-\gamma) Y|} .
\end{array}
$$

These bounds would suggest, and inspection of the convergence conditions for the various series employed will confirm, that as $Y$ becomes larger, the range of validity of the approximations (11.1)-(11.4) decreases. For the values of $\gamma$ and $Y$ occurring in physical fluids other than mercury, however, the smallest of the quantities (11.5) does not fall below $\frac{1}{2}$, so for values of $X$ below 0.1 the approximations (11.1) through (11.4) may be used with confidence. For all the weak and moderate conductors for which exact solutions are tabulated in \$21, the approximations (11.1) through (11.4) yield a maximum error of $10 \%$ when $X \leqq \frac{1}{3}$; for strong conductors, however, their error in every case but one is greater than $10 \%$ when $X=0.1$.

Dispersion. From (11.1) follows the classical result of Stokes and Stefan: there is no observable dispersion at frequencies such that $X^{2} \ll 1$. The known results $(4.11)_{1}$ and (4.36) $)_{1}$ are included in (11.1) as special cases. Almost by inspection, moreover, follows the new and interesting

First dispersion theorem. At moderately low frequencies, the dispersion of type $I$ waves is anomalous if $\gamma \geqq \frac{7}{3}$; if $\gamma<\frac{7}{3}$, then the dispersion is

$$
\left.\begin{array}{l}
\text { anomalous } \\
\text { neutral }
\end{array}\right\} \text { if }\left\{\begin{array}{r}
Y<\frac{5+2 \sqrt{\frac{4 \gamma-1}{\gamma-1}}}{7-3 \gamma} \\
Y=\frac{5+2 \sqrt{\frac{4 \gamma-1}{\gamma-1}}}{7-3 \gamma} \\
Y>\frac{5+2 \sqrt{\frac{4 \gamma-1}{\gamma-1}}}{7-3 \gamma}
\end{array}\right.
$$

It is interesting to examine the regions in the $\gamma-Y$ plane where dispersion is of one or the other type. The results, which are left to the reader to verify, are shown in Fig. 11.1. Included is the fact that in weak and submoderate conductors, the dispersion at moderate frequencies is always anomalous. Normal dispersion is 
to be expected mainly in strong conductors. When $\gamma$ is near to 1 , the value of $Y$ yielding neutral dispersion is quite sensitive to small changes in $\gamma$. This theorem gives precise form to the expectation, encountered in $\$ 4$, that sufficiently strong

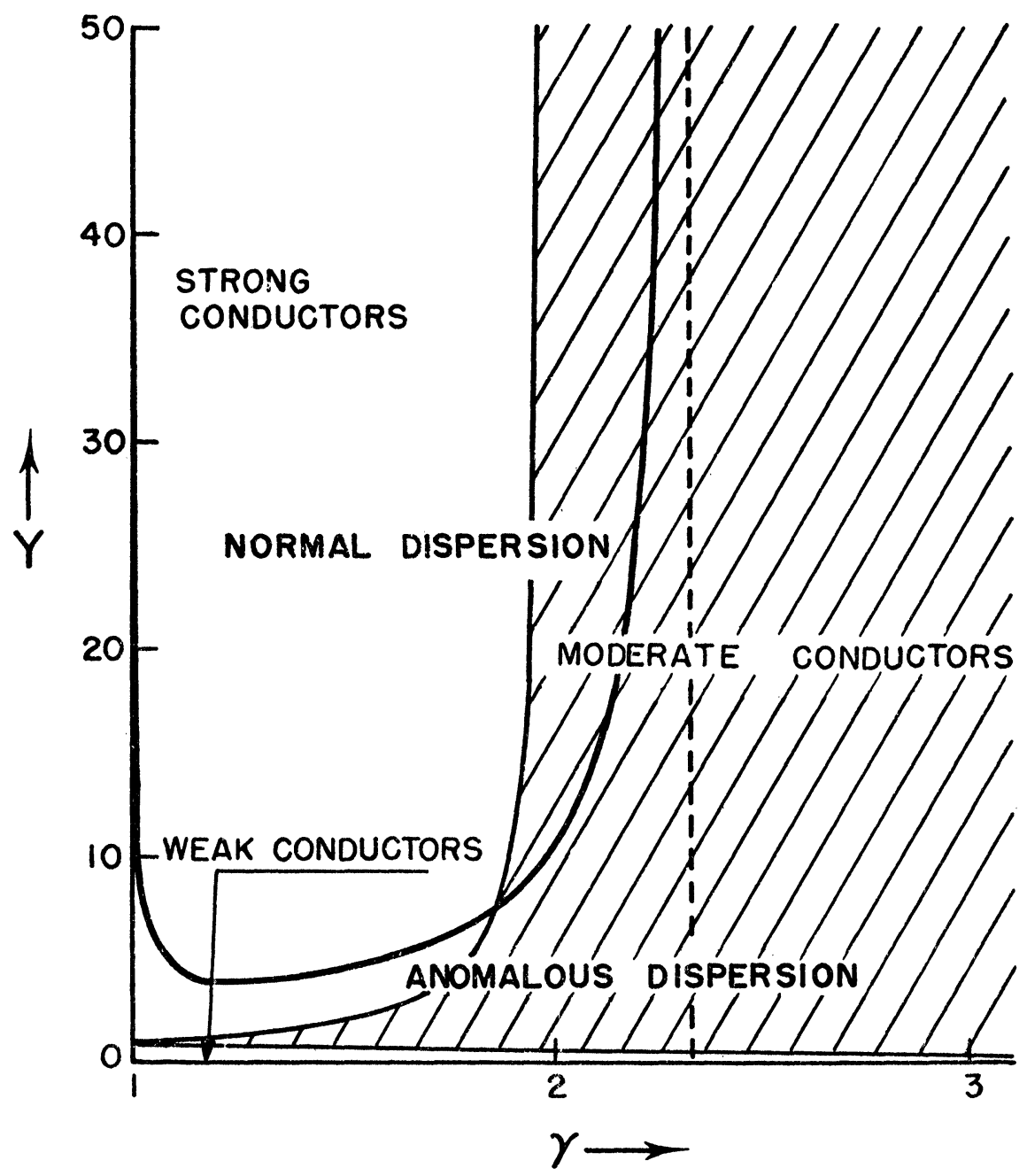

Fig. 11.1

The two types of dispersion at moderate frequencies

heat conduction can diminish the speed of sound at moderate frequencies to a value less than Laplace's.

Absorption. From any of (11.2) through (11.4) we may read off the classical 
result of Kirchhoff: at the lowest frequencies, the effect of heat conduction is to increase the absorption coefficient in the ratio $1+(\gamma-1) Y$. The next terms indicate, however, the following

Theorem of decreasing absorption. For all fluids, at moderate frequencies Kirchhoff's value for the absorption coefficient, $\pi X[1+(\gamma-1) Y]$, is too large, both for $A$ and for $A_{0}$. The proof consists in showing that the coefficients of $X^{2}$ in (11.3) and (11.4) are negative, whatever the values of $\gamma$ and $Y$, a straightforward but tedious matter which will be omitted here.

Measured absorption coefficients are often larger than the "classical" value $\frac{4}{3} \pi S[1+(\gamma-1) Y]$ but this disagreement may simply indicate that for the fluid in question $V>\frac{4}{3}$. On the other hand the theorem states that when the proper value for $V$ is employed, the absorption curve always lies below its tangent at the origin. Thus in the measurements reported to be in agreement with the "classical" value there is a possibility of compensating errors, resulting from taking too small a value, viz., $\frac{4}{3}$, for $\mathcal{V}$, but neglecting the non-linearity of the absorption curve. This suspicion is increased by the occasional reports of measured values less than the "classical."

One may notice also that the remark of Gurevich $(\$ 4)$ that the hydrodynamical theory in the piezotropic case predicts the simultaneous onset of dispersion and of non-linear absorption cannot be extended to the entire tri-variate case. In the strong conductor $\langle 1.15,10\rangle$, for example, the dispersion for $X \leqq \frac{1}{2}$ is very slight, while $A_{0}$ falls off sharply from the Kirchhoff linearization.

For type II waves we similarly obtain

$$
\begin{aligned}
r^{\prime} / r_{p}^{\prime} & =1+(\gamma-1)(1-Y)\left\{X+\frac{1}{2} X^{2}[\gamma+1-(3-\gamma) Y]+O\left(X^{3}\right)\right\} \\
\alpha^{\prime} / Q_{p}^{\prime} & =1-(\gamma-1)(1-Y)\left\{(\gamma-Y) X^{2}+O\left(X^{4}\right)\right\} \\
A_{0}^{\prime 2} / A_{0 p}^{\prime 2} & =1+(\gamma-1)(1-Y)\left\{X+O\left(X^{2}\right)\right\} \\
A^{\prime} / A_{p}^{\prime} & =1+(\gamma-1)(1-Y)\left\{X+O\left(X^{2}\right)\right\}
\end{aligned}
$$

where the measures with subscript $p$ are given by (4.23). The first terms will give very accurate results if $X$ be greatly inferior not only to the quantities (11.5) but also to

$$
\frac{1}{\gamma Y}, \quad \frac{1}{|1-Y||1-(2-\gamma) Y|} \text {. }
$$

Inspection of these formulae yields the

Low frequency approximation theorem for type II waves. At low and moderately low frequencies, the measures for type II waves for the fluid $\langle\gamma, Y\rangle$ are closely approximated by the corresponding measures for the piezotropic fluid $\langle 1, Y\rangle$. For the measures $r^{\prime}, A_{0}^{\prime}$, and $A^{\prime}$, the approximation so obtained is in the case of weak 
and submoderate conductors too small; in the case of strong and supermoderate conductors, too large.

The foregoing result is not important, however, since the relative measures are, with error $O\left(X^{2}\right)$,

$$
\Omega / 2 X Y=\frac{1}{2}\left\{[1+(\gamma-1) Y]^{2} X^{3} Y\right\} Q=1+(\gamma-1)(1-Y) X
$$

(For all the weak and moderate conductors for which the exact solutions are tabulated in \$21, these approximations yield a maximum error of $10 \%$ when $X \leqq 0.1$; for strong conductors, however, their error in every case exceeds $10 \%$ when $X=0.1$.) From the fact that (11.11) 1 yields $Q=\infty$ when $X=0$ we have the classical result of Kirchhoff : in all fluids, at sufficiently low frequencies type II waves will generally fail to be observed, since they are far more strongly absorbed than are type I waves. However, we note that since $Q \propto X^{-\frac{\pi}{2}}$, as $X$ increases $Q$ falls off very sharply, so that at moderate frequencies the possibility of observing type II waves increases with frequency. This extremely interesting result immediately raises the question, foreshadowed in $\$ 8$, of what happens to $Q$ at higher frequencies, a question which will be discussed int he next two sections.

The relative dispersion $\Omega$ increases with frequency at low frequencies, for all fluids: as frequency increases, type II waves tend to follow type I waves more closely. At moderate frequencies the dispersion of type II waves in all fluids is anomalous.

Inspection of (11.8) through (11.11) shows that the second term in all of them arises solely from the second term in (11.6), i.e. from the nature of the dispersion of type II waves. In particular we note the

Low frequency approximation theorem for the relative measures. At low frequencies $\Omega=\Omega_{p}+O(X)$, for any fluid. To approximate $\Omega$ and $Q$ by their asymptotic forms as $X \rightarrow 0$ gives values which at moderate frequencies are too small in the case of weak and submoderate conductors; too large in the case of strong and supermoderate conductors.

This result is significant because it reinforces the suggestion already drawn from the piezotropic case that in strong and supermoderate conductors, where $Y$ exceeds 1 , the possibility of observing type II waves is far greater than in weak or submoderate conductors, where $Y<1$.

12. Approximation for very high and moderately high frequencies. By straightforward but laborious calculations we obtain the following results for type I waves, for both weak and strong conductors, in the limit as $X \rightarrow \infty$ :

$$
a=\frac{\pi}{X}\left[1+\frac{(1-Y)\left(1-2 Y+\gamma Y^{2}\right)}{(1-\gamma Y)^{3} X^{2}}+O\left(\frac{1}{X^{4}}\right)\right]
$$

while, with error $O\left(X^{-2}\right)$,

$$
\frac{r}{2 X}=\frac{A}{2 \pi}=\frac{A_{0}^{2} X}{2 \pi^{2}}=1-\frac{1-Y}{(1-\gamma Y) X} .
$$


In order that the remainder terms be negligibly small, it is sufficient that $X$ greatly exceed the quantities

$$
\begin{array}{r}
\frac{1}{|1-\gamma Y|}, \frac{\sqrt{2} \sqrt{\left|1-2(4-3 \gamma) Y+\left(8-8 \gamma+\gamma^{2}\right) Y^{2}\right|}}{(1-\gamma Y)^{2}}, \frac{|1-Y|}{|1-\gamma Y|} \\
\frac{2 \sqrt{(\gamma-1) Y|1-Y|}}{(1-\gamma Y)^{2}}, \frac{\sqrt{|1-\gamma|\left|1-(3-\gamma) Y+\gamma Y^{2}\right|}}{\sqrt{2}|1-\gamma Y|^{3}}
\end{array}
$$

The accuracy of (12.2) is quite good near $Y=0$ but poor for larger $Y$. Comparison with the exact values in $\$ 21$ shows such irregularity as to defy a simple summary. As examples we mention that for $Y=.5$ and $Y=.8$, for all tabulated $\gamma,(12.2)$ is generally in error by more than $10 \%$ at $X=5$; while for several strong conductors the error in $A_{0}$ is less than $10 \%$ when $X \geqq 1$.

By inspection of (12.1) and (12.2) we obtain a number of theorems regarding type I waves in weak or strong conductors:

1. The dispersion approaches $\infty$ with $X$; hence in particular for sufficiently high frequencies the dispersion is always anomalous.

2. The true absorption coefficient $A$ approaches the limit $2 \pi$.

3. Both $a$ and $A_{0}$ approach 0 ; hence each experiences at least one maximum. (General proof of the phenomenon of visco-thermal resonance.)

4. All absorption and dispersion measures for type I waves approach asymptotically the values of the corresponding measures for pressure waves in a piezotropic fluid, values independent of $Y$.

This last result shows that at sufficiently high frequencies pressure waves in a piezotropic fluid furnish a first approximation to type I waves, for both weak and strong conductors. The degree of this approximation will be discussed presently, as soon as we consider the second terms in themselves.

Now in a weak conductor necessarily $Y<1$, while in a strong conductor necessarily $Y>1$. Also $1-2 Y+\gamma Y^{2}>0$ when $\gamma>1$. Thus the coefficient of the second terms in (12.1) and (12.2) is negative in both cases:

5. In both weak and strong conductors which are not piezotropic, all the absorption and dispersion measures approach their asymptotic values from below.

To compare the results with those for piezotropic fluids, we notice that with error $O\left(X^{-2}\right)$

$$
\frac{r}{r_{p}}=\frac{A}{A_{p}}=\frac{A_{0}^{2}}{A_{0 p}^{2}}=1-\frac{(\gamma-1) Y}{(1-\gamma Y) X}
$$

The sign of the correction term is negative if $\gamma Y<1$, positive if $\gamma Y>1$. Hence:

6. In weak conductors at high frequencies the measures $r, A, A_{0}$ are smaller than those for a piezotropic fluid; in strong conductors, greater.

Furthermore the correction term itself diminishes both as $Y \rightarrow 0$ when $Y$ is small and as $Y \rightarrow \infty$ when $Y$ is large:

7. For moderately high frequencies the piezotropic fluid serves a good approxima- 
tion, as far as type I waves are concerned, both for very slightly and for very strongly conducting fluids.

For type II waves we similarly obtain

$$
A^{\prime}=\frac{\pi}{\gamma Y X}\left\{1+\frac{(\gamma-1)\left(\gamma^{2} Y-2 \gamma Y+1\right)}{\gamma^{2}(1-\gamma Y)^{3} X^{2}}+O\left(\frac{1}{\bar{X}^{4}}\right)\right\},
$$

while with error $O\left(X^{-2}\right)$

$$
\frac{r^{\prime}}{2 \gamma Y X}=\frac{A^{\prime}}{2 \pi}=\frac{A_{0}^{\prime 2} \gamma Y X}{2 \pi^{2}}=1+\frac{\gamma-1}{\gamma(1-\gamma Y) X} .
$$

For the remainder terms to be negligible it is sufficient that $X$ greatly exceed not only the quantities (12.3) but also

$$
\frac{\sqrt{\gamma-1} \sqrt{1-2 \gamma(2-\gamma) Y-(\gamma-1) \gamma^{2} Y^{2}}}{\gamma(1-\gamma Y)^{2}}
$$

Here the remarks following (12.3) may be repeated, with the addition that for all strong conductors for which there are tabulated solutions in $\$ 21$ the error made by (12.6) is less than $10 \%$ for $X \geqq 0.1$, indeed often less than $1 \%$.

For the relative measures we have, with error $O\left(X^{-2}\right)$,

$$
\frac{\Omega}{\gamma Y}=\gamma Y Q=1+\frac{2-\frac{1}{\gamma}-Y}{(1-\gamma Y) X}
$$

or, alternatively,

$$
\frac{\Omega}{\gamma \Omega_{p}}=\frac{\gamma Q}{Q_{p}}=1+\frac{1-\frac{1}{\gamma}+\gamma Y}{(1-\gamma Y) X} .
$$

For all strong conductors for which there are tabulated solutions in $\$ 21$, the above formulae are accurate to within $10 \%$ when $X \geqq 4$; but for weak conductors, larger values of $X$ are required.

From (12.6) and (12.8) we conclude the

High frequency approximation theorem for type II waves. In the strong or weak conductor $\langle\gamma, Y\rangle$ at sufficiently high frequencies, $r^{\prime}, A^{\prime}, A_{0}^{\prime}, \Omega$, and $Q$ may be approximated by their values for the piezotropic fluid $\langle 1, \gamma Y\rangle$, the results being for weak conductors too small, for strong conductors too large.

We conclude also

8. For both weak and strong conductors, the ultimate ratio of speeds of propagation for the two types of waves is $\sqrt{\gamma Y}$ while the ultimate ratio of absorption co. efficients is $1 / \sqrt{\gamma Y}$.

9. In weak conductors at high and moderately high frequencies type II waves travel more slowly and are more strongly absorbed than are type I waves. 
When $X \leqq 5$ the increase given by the second term in (12.8) is not less than $20 \%$, so that at moderately high frequencies $\Omega$ and $Q$ are well above their asymptotic values.

For strong conductors $\gamma Y>1$ and hence, as was foreshadowed by the piezotropic case, Kirchhoff's conclusion of $\$ 11$ is reversed:

10. At sufficiently high frequencies in strong conductors, type I waves travel more slowly and are more strongly absorbed than are type II waves. Hence in strong conductors at very high frequencies it is type II waves, not type I waves, which are more easily observed.

The second term in (12.8) is never greater than $(20 / \gamma) \%$ when $X=5$, so that for strong conductors the asymptotic values of $\Omega$ and $Q$ are more rapidly approached than for weak conductors.

Thus far in the present section moderate conductors have been excluded. For them it is possible by considering the power series expansions of (10.2) and (10.3) about $X=\infty$ to prove the following

Interchange theorem. In moderate conductors at sufficiently high frequencies, the two leading terms in the formal power series expansions in $1 / X$ for the absorption and dispersion measures coincide with those for strong or weak conductors, except that the assignment of wave types is interchanged.

Thus for moderate conductors primes should be dropped from (12.5) and (12.6) and supplied for (12.1) and (12.2), while the relative measures are the reciprocals of (12.8), viz., with error $O\left(X^{-2}\right)$,

$$
\gamma Y \Omega=\frac{Q}{\gamma Y}=1+\frac{2-\frac{1}{\gamma}-Y}{(\gamma Y-1) X} .
$$

For moderate conductors the adjusted formulae (12.2) and (12.6b) yield good results for $Y$ near to 1, but poor values for $Y$ near $\gamma^{-1}$ or $(2-\gamma)^{-1}$. For example, for $\left\langle\frac{5}{3}, 1.05\right\rangle$ and $\left\langle\frac{5}{3}, 1.25\right\rangle$ their error is less than $10 \%$ when $X \leqq 2.5$. Values of $X$ in excess of 5 are required in order to obtain similar accuracy from (12.10).

From the interchange theorem we conclude

11. In the moderate conductor $\langle\gamma, Y\rangle$ at high frequencies, the measures for type $I$ waves may be approximated by those for thermal waves in the piezotropic fluid $\langle 1, \gamma Y\rangle$, the values so obtained for $r, A$, and $A_{0}$ being somewhat too large. The measures for type II waves may be approximated by those for pressure waves in a piezotropic fluid, the values so obtained for $r^{\prime}, A^{\prime}$, and $A_{0}^{\prime}$ being somewhat too small. Thus at sufficiently high frequencies type II waves travel more slowly and are more strongly absorbed than are type I waves.

This result illustrates the discontinuous nature of the separation between strong conductors and other fluids. Suppose, for example, that $\gamma$ is fixed at a value $1<\gamma<2$, and let $Y$ increase. As $Y$ rises from 0 to $1 / \gamma$ the fluid $\langle\gamma, Y\rangle$ remains in the class of weak conductors, and the asymptotic value $Q_{a}$ of $Q$ decreases steadily from $\infty$ to 1 . As $Y$ rises from $1 / \gamma$ to $(2-\gamma)^{-1}-0$ the fluid crosses the moderate conductor region and approaches the singular $\mathrm{A}$ line; 
meanwhile $Q_{a}$ increases steadily from 1 to $\gamma(2-\gamma)^{-1}$, a value which when $\gamma$ is near to 2 is again very large. Now let $Y$ jump from $(2-\gamma)^{-1}-0$ to $(2-\gamma)^{-1}+$ 0 , thus crossing the singular A line; then $Q_{a}$ decreases discontinuously from $\gamma(2-\gamma)^{-1}$ to $(2-\gamma) / \gamma$ :

$$
\left[Q_{a}\right]=-\frac{2(\gamma-1)}{\gamma(2-\gamma)}
$$

As $\gamma \rightarrow 1+0$, this jump approaches 0 , but as $\gamma \rightarrow 2-0$, it approaches $-\infty$. Finally, as $Y$ increases from $(2-\gamma)^{-1}+0$ to $\infty, Q_{a}$ decreases from $(2-\gamma) / \gamma$ to 0 .

Instead, we may hold $Y$ fixed and let $\gamma$ increase. If $0<Y<1$, the fluid $\langle\gamma, Y\rangle$ simply moves from the weak conductor region into the submoderate conductor region. $Q_{a}$ decreases from $1 / Y$ to 1 as singular B line is crossed, then increases again from 1 to $\infty$. If $Y>1$, the fluid moves from the strong conductor region into the supermoderate conductor region. $Q_{a}$ decreases from $1 / Y$ to $(2 Y-1)^{-1}$, then as the singular A line is crossed it increases discontinuously to $2 Y-1$ :

$$
\left[Q_{a}\right]=+\frac{Y-1}{2 Y-1} .
$$

When $Y$ is near to 1 , this jump is small, but for large $Y$ it approaches $+\infty$. As $\gamma$ continues to increase, $Q_{a}$ increases from $2 Y-1$ to $\infty$.

Similarly interesting results may be obtained for dispersion.

Interpretation of the fact that $r \sim 2 X, r^{\prime} \sim 2 \gamma Y X$ as $X \rightarrow \infty$ for weak and strong conductors, together with the interchange theorem, yields the

Generalized Gurevich theorem. In all fluids, the asymptotic speed of each type of wave as $X \rightarrow \infty$ is independent of the form of the equation of state, being determined either by viscosity alone or by thermal conductivity alone. For weak or strong conductors

$$
V^{2} \sim \frac{2 \omega(\lambda+2 \mu)}{\rho}, \quad V^{\prime 2} \sim \frac{2 \gamma \kappa \omega}{\rho c_{p}}
$$

alternatively expressed, the asymptotic speeds are the same as for pressure waves and thermal waves, respectively, in the piezotropic fluid $\langle 1, \gamma Y\rangle$. For moderate conductors, the expressions for $V$ and $V^{\prime}$ are to be interchanged.

Corollary. In any viscous fluid the speed of sound increases without limit at sufficiently high frequencies.

As remarked in $\$ 4$, this corollary fails when $Y=\infty$.

By noting that $r^{\prime} \sim r$ if and only if $\gamma Y=1$, and also that $Q \rightarrow 1$ if and only if $\gamma Y=1$, we obtain the following

Characterization of the singular fluids $B$. A necessary and sufficient condition that at large frequencies the two types of waves travel at equal speeds and suffer equal absorption is that the fluid be one of the singular fluids $B$. 
Finally, from (11.9), $(12.2)_{2}$ and $(12.6 \mathrm{~b})_{2}$, taking account of the interchange theorem, we have the

Theorem of the true absorption coefficient. In any fluid the true absorption coefficient per wave length for either type of wave approaches $2 \pi$ with infinite frequency. Moreover, the coefficient $A^{\prime}$, beginning with the value $2 \pi$ when $X=0$, in a weak or submoderate conductor must experience at least one maximum; in a strong or supermoderate conductor, at least one minimum.

13. Summary and conjectures. The physical occurrence of the two types of waves. Those of the conclusions from the formulae proved in \$\$11-12 which hold in simple form for all fluids, without subdivision into types, will now be assembled. These, taken together with numerical exploration of special cases, make plausible the conjectures stated immediately below. The symbol " $\precsim$ " is to mean "is less than and is asymptotic to"; " $\succsim$ " is defined analogously. $A_{K}$ stands for Kirchhoff's absorption coefficient (4.20).

Theorem 1. As $X \rightarrow 0, A_{0} \lesssim A_{K} ; A_{0}$ experiences at least one maximum; as $x \rightarrow \infty, A_{0} \rightarrow 0$.

Conjecture 1. A0 experiences exactly one maximum.

Theorem 2. As $X \rightarrow 0, A \lesssim A_{K}$; as $X \rightarrow \infty, A \lesssim 2 \pi$.

Conjecture 2. $A$ is a monotone increasing function of $X$.

Theorem 3. As $X \rightarrow 0, r \rightarrow 1$; as $X \rightarrow \infty, r \rightarrow \infty$.

Conjecture 3. The curve of $r$ against $X$ is curved positively upward at all points.

Theorem 4. As $X \rightarrow 0, A_{0}^{\prime 2} \sim 2 \pi^{2} /(\gamma Y X) \rightarrow \infty$; as $X \rightarrow \infty, A_{0}^{\prime} \rightarrow 0$.

Conjecture 4. $A_{0}^{\prime}$ is a monotone decreasing function of $X$.

Theorem 5. As $X \rightarrow 0$ and as $X \rightarrow \infty, A^{\prime} \rightarrow 2 \pi$.

Conjecture 5. $A^{\prime}$ is closely approximated by $2 \pi$ except in narrow frequency bands about such extremes as it possesses.

Theorem 6. As $X \rightarrow 0, r^{\prime} \sim r_{p}^{\prime} \rightarrow 0$; as $X \rightarrow \infty, r^{\prime} \rightarrow \infty$.

Conjecture 6. $r^{\prime}$ is a monotone increasing function of $X$.

Theorem 7. As $X \rightarrow 0, \Omega \sim \Omega_{p} \rightarrow 0$; as $X \rightarrow \infty, \Omega \rightarrow$ a finite limit.

Conjecture 7. $\Omega$ experiences a single maximum.

Theorem 8. As $X \rightarrow 0, Q \sim A_{0 p}^{2} / A_{k}^{2}=Q_{p}^{2} /[1+(\gamma-1) Y]^{2}$; as $X \rightarrow \infty$, $Q \rightarrow$ a finite limit.

Conjecture 8. $Q$ is a monotone decreasing function of $X$.

From theorem 8 , were conjecture 8 to be established rigorously, we should be able to draw the following

Major relative absorption corollary. In weak conductors we have $Q>(\gamma Y)^{-1}>$ 1. In moderate conductors we have $Q>\gamma Y>1$. But in strong conductors there exists a single dividing frequency number $X_{d}$ such that $Q>1$ when $X<X_{d}, Q<1$ when $X>X_{d}$.

Adopting the viewpoint of Kirchhoff that it is the less absorbed of the two types of waves which in general will be observed, we should then conclude that in both weak and moderate conductors, it is the measures for type I waves which are physically relevant at all frequencies; in strong conductors, however, this con- 
clusion holds only when $X \ll X_{d}$, while when $X \gg X_{d}$ it is the measures for type II waves which become physically relevant, and, finally, when $X \approx X_{d}$ a sinusoidal driving force will fail to produce simple sinusoidal waves of either type.

14. A power series expansion and its consequences. We shall explore a new view of the subject. We may write (9.2) in the form

$$
\begin{array}{rl}
-2\left(\frac{2 \pi}{\sigma}\right)^{2}=1+i & X(1+\gamma Y) \\
+ & {[1+i X(1-\gamma Y)]\left\{1+\frac{4 i(\gamma-1) X Y}{[1+i X(1-\gamma Y)]^{2}}\right\}^{\frac{1}{2}} .}
\end{array}
$$

Since for complex numbers in general $\sqrt{z w} \neq \sqrt{z} \sqrt{w}$, we cannot at once identify the two determinations of the root occurring in (14.1) with those employed in the principal definition of $\$ 9$. However, for sufficiently small $X$ the principal determination in (14.1) is given by the usual series, and comparison of the leading terms with those in the series (not written down in this paper) from which (11.1) and (11.2) were calculated shows that in all types of fluids, for sufficiently small $X$ the principal determination in (14.1) yields type I waves. But each determination is an analytic function of the argument $w$ of the root when this latter varies over a cut plane. Hence if $w$ travels within a proper cut plane as $X$ varies from 0 to $\infty$, the agreement between these determinations and those of $\$ 9$ may be continued over this whole plane.

Now we have

$$
w=1+\frac{2 i M Q}{(1 \pm i \vartheta)^{2}}, \quad \text { where } \quad M \equiv \frac{2(\gamma-1) Y}{|1-\gamma Y|}
$$

(9.3) $)_{1}$ is employed, and the plus sign applies to weak conductors, the minus sign to moderate and strong conductors. Hence for a particular fluid $\langle\gamma, Y\rangle$, as $X$ varies from 0 to $\infty$, so does the parameter $\mathcal{Q}$, and the variable $w=u+i v$ describes the curve

$$
u=1 \pm \frac{4 M Q^{2}}{\left(1+Q^{2}\right)^{2}}, \quad v=\frac{2 M Q\left(1-Q^{2}\right)}{\left(1+Q^{2}\right)^{2}}
$$

For any tri-variate non-singular fluid, this curve is a circle with center at $(1 \pm$ $\left.\frac{1}{2} M, 0\right)$ and radius $\frac{1}{2} M$. The initial point, corresponding to zero frequency $(Q=0)$ is $w=1$. As $Q$ increases, the upper half of the circle is traversed, until at $Q=1$ the circle crosses the real axis, returning again to $w=1$ when $Q=\infty$. For weak conductors the circle lies to the right of $w=1$, while for moderate and strong conductors it lies to the left. In the latter case it crosses the real axis at $u=$ $1-M$. Now for both strong and moderate conductors we have $1-M=\frac{1}{\gamma Y-1}[(2-\gamma) Y-1] \begin{cases}<0 & \text { for moderate conductors } \\ >0 & \text { for (tri-variate) strong conductors. }\end{cases}$ 
In fact, for tri-variate strong conductors $1>M>0$; for moderate conductors $\infty>M>1$; while for weak conductors $0 \leqq M<\infty$. Hence for tri-variate strong conductors the circle again lies entirely in the cut plane, but for moderate conductors it crosses the cut when $Q=1$. The three cases are illustrated in Fig. 14.1. For moderate conductors $\sqrt{w}$ jumps from $i \sqrt{M-1}$ to $-i \sqrt{M-1}$ at
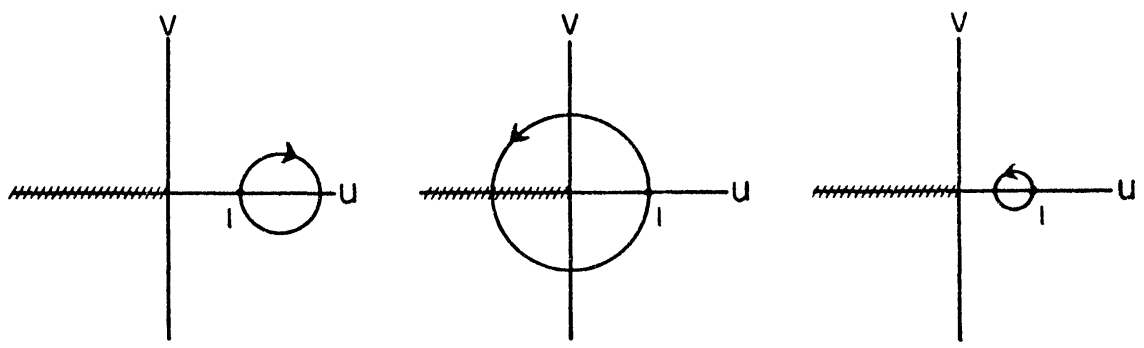

FIG. 14.1

Argument curves for the root in (14.1)

(a) weak conductors (case drawn: $M=1$ ) (b) moderate conductors (case drawn: $M=2$ ) (c) strong conductors (case drawn: $M=\frac{1}{2}$ )

$Q=1$, and hence its product by $1+i X(1-\gamma Y)$ jumps from $(1+i) \sqrt{M-1}$ to $-(1+i) \sqrt{M-1}$, or, equivalently, from $\sqrt{z}$ to $-\sqrt{z}$. Summarizing these results, we have the following

Branch theorem. For weak and strong conductors alike, the principal determination of the square root in (14.1) yields absorption and dispersion measures for type I waves at all frequencies. For moderate conductors the principal determination yields

$$
\left.\begin{array}{l}
\text { type I waves } \\
\text { type II waves }
\end{array}\right\} \quad\left\{\begin{array}{l}
X<\frac{1}{\gamma Y-1} \\
X>\frac{1}{\gamma Y-1}
\end{array} .\right.
$$

As was mentioned in $\$ 4$, various investigators have used power series expansions for the roots $\sigma$ in a rather loose way. The foregoing analytical theorem enables us now to found the series method upon a rational basis.

The circle of convergence $|w|<1$ for the binomial expansion of the root in (14.1) is equivalent to the region

$$
\frac{2 M Q}{1+Q^{2}}<1
$$

which is shown in Fig. 14.2. Analysis of this relation yields the

Convergence theorem. For any fluid $\langle\gamma, Y\rangle$, i.e., for any value of $M$, there exist frequencies for which the power series expansion of the radical in (14.1) converges. 
1. For weak conductors such that $M<1$ (equivalently, $\left.Y<(3 \gamma-2)^{-1}\right)$, and for all strong conductors, the series converges for the entire frequency range and yields the measures for type $I$ waves. (The appropriate region of the $\gamma-Y$ plane is shown in Fig. 14.3.)

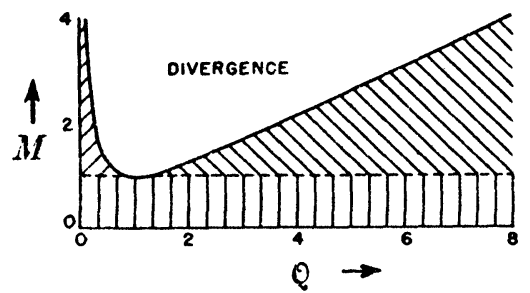

FIG. 14.2

Convergence region for the series expansion.

Vertical lining: weak or strong conductors, type I waves.

Slant lining at left: weak or moderate conductors, type I waves.

Slant lining at right: type $I$ waves if weak conductor, type II waves if moderate.

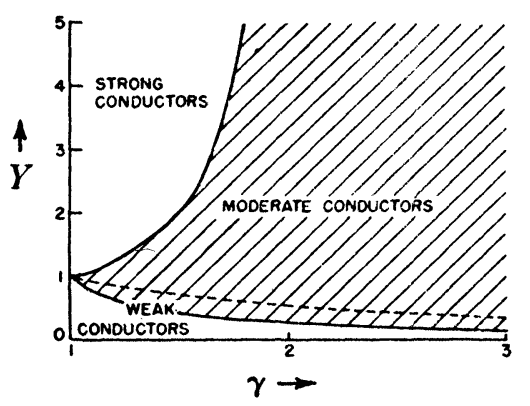

Frg. 14.3

The region $M<1$ (not shaded), in which the series converges for all $X$.

2. For weak conductors such that $M \geqq 1$, and for all moderate conductors, the series converges both in the low frequency range

$$
Q<M-\sqrt{M^{2}-1}
$$

and in the high frequency range

$$
Q>M+\sqrt{M^{2}-1}
$$

For weak conductors the series yields the measures for type I waves in both the high and the low frequency range. For moderate conductors it yields the measures for type I waves in the low frequency range; those for type II waves in the high frequency range.

For any fluid, then, the method of expansion in powers of the frequency may be applied at sufficiently low frequencies and at sufficiently high frequencies. However, some of the statements in the literature concerning asymptotic values of the measures are partially incorrect from disregard of the fact that for moderate conductors at high frequencies the result yields the measures for type II waves, not type I waves. The convergence theorem stated above replaces the special results and suspicions on this subject which have occurred in earlier works (see §4). The leading terms in the resulting expansions for the absorption and dispersion measures have already been obtained in a different way in $\$ \$ 11-12$. 
The interchange theorem of $\$ 12$ is an evident corollary of the convergence theorem. The remainder of this section will discuss the expansions obtainable under the weaker assumption (14.4).

Putting $U \equiv M Q /\left(1+Q^{2}\right)$, we have from (14.1)

$$
\begin{aligned}
-2\left(\frac{2 \pi}{\sigma}\right)^{2}=1+ & i X(1+\gamma Y) \\
& \pm\left\{1+i X(1-\gamma Y)+\frac{2 i(\gamma-1) X Y}{1+i X(1-\gamma Y)}\right\}+O\left(U^{2}\right)
\end{aligned}
$$

where the signs are to be selected in accordance with the branch theorem. $O\left(U^{2}\right)$ is to be interpreted with respect to the limit $U \rightarrow \infty$ while $\gamma$ and $\mathcal{Q}$ are held fixed.

Taking the upper sign yields

$$
G=1 \pm \frac{1}{2} \Theta U+O\left(U^{2}\right), \quad H=X+\frac{1}{2} U+O\left(U^{2}\right),
$$

while taking the lower sign yields

$$
G=\mp \frac{1}{2} \Theta U+O\left(U^{2}\right), \quad H=\gamma Y X-\frac{1}{2} U+O\left(U^{2}\right) .
$$

After some labor we obtain for weak or strong conductors the following results, ${ }^{1}$ in which $M, Q$, and $U$ have been replaced by their expressions in terms of $\gamma, Y$, and $X$ :

$$
\begin{gathered}
\frac{a}{a_{p}}=1+(\gamma-1) Y \frac{1-(3-2 \gamma Y) X^{2}}{\left(1+X^{2}\right)\left(1+X^{2}[1-\gamma Y]^{2}\right)}, \\
\frac{r}{r_{p}}=1+(\gamma-1) Y X^{2} \frac{3-\gamma Y+(2-\gamma Y) \sqrt{1+X^{2}}-(1-\gamma Y) X^{2}}{\left(1+X^{2}\right)\left(1+X^{2}[1-\gamma Y]^{2}\right)\left(1+\sqrt{1+X^{2}}\right)} \\
\frac{A}{A_{p}}=1+(\gamma-1) Y \frac{1-X^{2}(1-\gamma Y)}{\sqrt{1+X^{2}\left(1+X^{2}[1-\gamma Y]^{2}\right)}}, \\
\frac{A_{0}^{2}}{A_{0 p}^{2}}=1+(\gamma-1) Y \frac{1-(3-2 \gamma Y) X^{2}+\left(1-X^{2}[1-\gamma Y]\right) \sqrt{1+X^{2}}}{\left(1+X^{2}\right)\left(1+X^{2}[1-\gamma Y]^{2}\right)} \\
\frac{\Omega}{\Omega_{p}}=\frac{r^{\prime}}{r_{p}^{\prime}} \\
=\frac{1+\gamma^{2} X^{2}(1-\gamma Y)^{2}}{\sqrt{1+X^{2}(1-\gamma Y)^{2}} \sqrt{1+\gamma^{2} X^{2}(1-\gamma Y)^{2}-(\gamma-1) X(1-\gamma Y)}}
\end{gathered}
$$

1 These results are correct as stated. They are not, however, precisely the leading terms in a series expansion in powers of $U$, since their form has been simplified by retaining explicitly certain quantities which could have been absorbed into the remainders. 


$$
\begin{aligned}
& \frac{Q^{\prime}}{Q_{0 p}^{\prime}}=\frac{1+\gamma X^{2}(1-\gamma Y)^{2}}{1+\gamma^{2} X^{2}(1-\gamma Y)^{2}} \\
& \frac{A^{\prime}}{A_{p}^{\prime}}=\frac{1+\gamma X^{2}(1-\gamma Y)^{2}}{\sqrt{1+X^{2}(1-\gamma Y)^{2}} \sqrt{1+\gamma^{2} X^{2}(1-\gamma Y)^{2}-(\gamma-1) X(1-\gamma Y)}}, \\
& \frac{Q}{Q_{p}}=\frac{A_{0}^{\prime 2}}{A_{0 p}^{\prime 2}} \\
& =\frac{\left[1+\gamma X^{2}(1-\gamma Y)^{2}\right] /\left[1+\gamma^{2} X^{2}(1-\gamma Y)^{2}\right]}{\sqrt{1+X^{2}(1-\gamma Y)^{2}} \sqrt{1+\gamma^{2} X^{2}(1-\gamma Y)^{2}-(\gamma-1) X(1-\gamma Y)}} .
\end{aligned}
$$

In (14.10)-(14.13) the error is $O\left(U^{2}\right)$, while on the right hand sides of (14.14)(14.17) a factor $\{1+O(U)\}$ is to be understood. For moderate conductors these results still hold when $X<(\gamma Y-1)^{-1}$; for $X>(\gamma Y-1)^{-1}$, primed and unprimed quantities are to be interchanged, while $\Omega$ and $Q$ are to be replaced by their reciprocals.

For any given fluid $\langle\gamma, Y\rangle$, these approximations are of course best for very high and for very low frequencies, since $U \rightarrow 0$ both as $X \rightarrow 0$ and as $X \rightarrow \infty$. They are poorest in the range where $U \approx 1$, or, equivalently, $X|1-\gamma Y| \approx$ $M \pm \sqrt{ } M^{2}-1$. However, for certain fluids $U$ is a rather small quantity at all frequencies, and for these fluids the results just obtained show precisely how the tri-variate character of the fluid $\langle\gamma, Y\rangle$ causes the absorption and dispersion curves to depart from those for the piezotropic fluid $\langle 1, Y\rangle$.

There are several broad observations:

1. The correction terms in $A$ and $A_{0}$ increase them from $A_{p}$ and $A_{0 p}$ in Kirchhoff's ratio $(\gamma-1) Y /[1+(\gamma-1) Y]$ at low frequencies.

2. The correction terms in (14.11)-(14.13) fall off to zero at $X=\infty$ with $X^{-1}$.

3. The effect of the correction terms in (14.14)-(14.17) is roughly equivalent to a steady changeover with increasing frequency from the corresponding measures for the fluid $\langle 1, Y\rangle$ to those for the fluid $\langle 1, \gamma Y\rangle$.

We shall attempt to use the above approximate formulae only for very weak conductors and for very strong conductors, for which it will be legitimate to suppose $M \ll 1$, and hence $U \ll 1$. Consequently the approximate formulae are very accurate at all frequencies, rendering precise our earlier expectation that the measures for piezotropic fluids, providing the low frequency absorption coefficients be increased in Kirchhoff's ratio, serve as first approximations both to those for very weak and to those for very strong conductors. At the same time we have furnished a first order correction to this approximation. The qualitative pattern of absorption and dispersion of type I waves is then the same as for pressure waves in piezotropic fluids. We shall now consider in detail the first order quantitative changes.

Dispersion. The second term in (14.11) vanishes both for $X=0$ and for $X \rightarrow$ $\infty$. Examination of the sign of the numerator then yields the

Second dispersion theorem. Not only at very low frequencies but also at very 
high frequencies dispersion of type I waves both in very weak and in very strong conductors is virtually the same as for pressure waves in a piezotropic fluid. In a slightly conducting fluid $(M \ll 1, \gamma Y<1)$, dispersion of type I waves at low frequencies is a little greater than in a piezotropic fluid; at high frequencies, a little less. In a very strongly conducting fluid $\left(M \ll 1, \gamma<2, Y>(2-\gamma)^{-1}\right)$, dispersion of type $I$ waves at low frequencies is greater or less than in a piezotropic fluid, according as $\gamma Y<5$ or $\gamma Y>5$; at high frequencies, always greater.

Absorption. Both the measures $Q$ and $A_{0}$ possess single maxima, but the expressions for the corresponding resonant frequency numbers are rather elaborate and will not be written down here.

We confine ourselves to the remark that the peak in $A_{0}$ is very flat, so much so that a perturbation formula is worthless, even though the actual value of $A_{0}$ is given with great accuracy by (14.13), even near the peak. Moreover, experimental values of $A_{0}$ are often determined very inaccurately and plotted against $\log \omega$. On a rough graph of this type the correction in $X$ h afforded by a small amount or a great amount of heat conductivity would be slight, and it would seem that the piezotropic value $X$ 月 $=\sqrt{3}$ would be sufficiently accurate. For this value of $X$, the value of $A_{0}$ is for weak conductors decreased in the approximate ratio $1-\frac{3}{4}(\gamma-1) Y$ from $A_{0 p}$; for strong conductors, increased in the approximate ratio $1+(\gamma-1) Y / \gamma$. On the other hand, we can get a very good approximate formula for $X^{*}$, the resonant frequency number for $a$. Unfortunately, however, is not convenient for treatment of experimental data, since it requires measurements not only of absorption but also of dispersion, with consequent greater likelihood of error.

15. Second approximation for weak conductors. ${ }^{1}$ We have seen that piezotropic fluids in which $\mathrm{Y}<1$ serve as first approximations for weak conductors. To obtain a second approximation, let us assume

$$
Y \ll \min \left(\frac{1}{\gamma}, \frac{1}{2(\gamma-1)}\right),
$$

or, equivalently,

$$
\frac{1}{P} \ll v \min \left(\frac{1}{\gamma}, \frac{1}{2(\gamma-1)}\right) .
$$

Since $v \geqq \frac{4}{3}$ always, while for most liquids $\gamma<1.2$, the condition (15.2) reduces to $P^{-1} \ll 1$ at worst. From Table 3.1 it appears that for most liquids $P^{-1}<.15$. Hence the assumption (15.1) will be satisfied by most liquids.

From (15.1) we have both $\gamma Y \ll 1$ and $\gamma Y \ll[2(\gamma-1)]^{-1}$ so that by $(14.2)_{1}$ we have $M \ll 1$. Consequently all the results of $\$ 14$ hold, with the further

\footnotetext{
1 These results were first published in my note, "Preliminary report: Non-linear absorption and dispersion of plane ultrasonic waves in pure fluids," J. Wash. Acad. 42, 3336 (1952).
} 
simplification that only terms of first order in $Y$ need be retained. For type I waves the following approximate measures result:

$$
\begin{aligned}
& \frac{a}{a_{p}} \approx 1+(\gamma-1) Y \frac{1-3 X^{2}}{\left(1+X^{2}\right)^{2}}, \\
& \frac{r}{r_{p}} \approx 1+(\gamma-1) Y X^{2} \frac{3+2 \sqrt{1+X^{2}}-X^{2}}{\left(1+X^{2}\right)^{2}\left(1+\sqrt{1+X^{2}}\right)}, \\
& \frac{A}{A_{p}} \approx 1+(\gamma-1) Y \frac{1-X^{2}}{\left(1+X^{2}\right)^{\frac{2}{2}}}, \\
& \frac{A_{0}^{2}}{A_{0 p}^{2}} \approx 1+(\gamma-1) Y \frac{1-3 X^{2}+\left(1-X^{2}\right) \sqrt{1-X^{2}}}{\left(1+X^{2}\right)^{2}} .
\end{aligned}
$$

Numerical comparison of these formulae with the exact values tabulated in $\$ 21$ indicates that they are accurate in a range beyond that which could reasonably be expected. For example, for $\langle 1.25, .25\rangle,\langle 1.40, .25\rangle$, and $\left\langle\frac{5}{3}, .25\right\rangle$ their maximum error is $4 \%$, and in many cases the error is less than $1 \%$; for $<1.40$, $.5\rangle$ it does not exceed $6 \%$, although for $\langle 1.25, .8\rangle$ it is considerably worse.

The resonant frequency number $X^{*}$ for $Q$ is easily shown to be given approximately by

$$
X^{*} \approx 1-\frac{1}{2}(\gamma-1) Y
$$

a formula which turns out to be extremely accurate. For example, for the case $\gamma=\frac{5}{3}, Y=1.05,(\gamma-1) Y=.70$, when the hypotheses under which it was derived are not correct at all, its error does not exceed $10 \%$. To determine $\lambda$ from (15.7) we have

$$
\frac{\lambda}{\mu} \approx-2+\frac{1+\sqrt{1-2(\gamma-1) S^{*} / P}}{2 S^{*}}
$$

or, more crudely,

$$
\frac{\lambda}{\mu} \approx-2+\frac{1}{S^{*}}-\frac{\gamma-1}{2 P}
$$

For the height of the peak we have

$$
Q^{*} \approx \frac{1}{2} \pi\left[1-\frac{1}{2}(\gamma-1) Y\right] .
$$

Thus the effect of heat conduction is to decrease both the resonant frequency and the height of the resonant peak.

16. Second approximation for strong conductors. We have seen that piezotropic fluids in which $\mathrm{Y}>1$ serve as first approximations for strong conductors. 
To obtain a second approximation, let us assume

$$
Y \gg \frac{1}{\gamma}, \quad \gamma-1 \ll \frac{1}{2} \gamma .
$$

Since $P^{-1}=38, \gamma=1.15$ for mercury, both these assumptions will be rather roughly satisfied, providing $v$ do not too greatly exceed $\frac{4}{3}$. From (16.1) follows $M \ll 1$, and the expansions of $\$ 14$ may be simplified by the approximation $\gamma Y-1 \approx \gamma Y$. Putting $W \equiv \gamma X Y$, we obtain

$$
\begin{aligned}
& \frac{Q}{Q_{p}} \approx 1+(\gamma-1) Y \frac{1+2 W X}{\left(1+X^{2}\right)\left(1+W^{2}\right)}, \\
& \frac{r}{r_{p}} \approx 1-\frac{\gamma-1}{\gamma} \frac{W^{2}}{\sqrt{1+X^{2}\left(1+W^{2}\right)}}, \\
& \frac{A}{A_{p}} \approx 1+\frac{\gamma-1}{\gamma} \frac{W^{2}}{\sqrt{1+X^{2}}\left(1+W^{2}\right)} \\
& \frac{A_{0}^{2}}{A_{0 p}^{2}} \approx 1+(\gamma-1) Y \frac{1+2 X W+(1-X W) \sqrt{1+X^{2}}}{\left(1+X^{2}\right)\left(1+W^{2}\right)} \\
& \frac{\Omega}{\Omega_{p}} \approx \frac{r^{\prime}}{r_{p}^{\prime}} \approx \frac{1+\gamma^{2} W^{2}}{\sqrt{1+W^{2} \sqrt{1+\gamma^{2} W^{2}}+(\gamma-1) W}}, \\
& \frac{Q^{\prime}}{Q_{p}^{\prime}} \approx \frac{1+\gamma W^{2}}{1+\gamma^{2} W^{2}}, \quad \\
& \frac{A^{\prime}}{A_{p}^{\prime}} \approx \frac{1+\gamma W^{2}}{\sqrt{1+W^{2} \sqrt{1+\gamma^{2} W^{2}+(\gamma-1) W}}} \\
& \frac{Q}{Q_{p}} \approx \frac{A_{0}^{\prime 2}}{A_{0 p}^{\prime 2}} \approx \frac{\left(1+\gamma W^{2}\right)^{2} /\left(1+\gamma^{2} W^{2}\right)}{\sqrt{1+W^{2}} \sqrt{1+\gamma^{2} W^{2}}+(\gamma-1) W} \cdot
\end{aligned}
$$

For all the strong conductors except $\langle 1.50,50\rangle$ for which tabulated values are given in $\$ 21$, the error of these approximations does not exceed $16 \%$; when $X>1$, it does not exceed $4 \%$; and for several fluids it is less than $\frac{1}{2} \%$ over much of the frequency range. In general, (16.8)-(16.9) are more accurate than (16.3)-(16.5).

For fluids in which $\gamma$ is fairly near to 1 , the denominators in (16.8) and (16.9) may be simplified by omitting the term $(\gamma-1) W$.

17. Moderate conductors. The duality theorem. From much of our discussion the moderate conductors have been excluded. To summarize what we have learned so far in a single phrase, the piezotropic fluids serve as first approximations both for weak conductors and for strong conductors. We show now that, 
properly employed, they furnish a similar first approximation for moderate conductors.

Returning to the basic equations (9.3) and (9.4), we begin by asking a general question: let $\sigma_{1}(Q), \sigma_{2}(Q)$ be the solutions for a given fluid $\langle\gamma, Y\rangle$; then do there exist constants $k, l, \bar{\gamma}, \bar{Y}$ such that $l \sigma_{1}(k \vartheta), l \sigma_{2}(k \vartheta)$ are solutions for the fluid $\langle\bar{\gamma}, \bar{Y}\rangle$ ?

To multiply $\sigma$ by $l$ is to multiply $G$ and $H$ by $1 / l$ and hence, by (1.16), to multiply $r$ by $1 / l$. But by (10.1), $r=1+O\left(X^{2}\right)$ as $X \rightarrow 0$ for all fluids, and hence we must have $l=1$.

Now proceed with (9.3) and (9.4). To obtain a positive answer to our question, we must have

$$
i K Q+\left(1-Q^{2}+2 i L Q\right)^{\frac{1}{2}}=i \bar{K} k Q+\left(1-k^{2} Q^{2}+2 i k \bar{L} Q\right)^{\frac{1}{2}},
$$

where $\bar{K}$ and $\bar{L}$ denote the expressions resulting from replacing $\gamma, Y$ by $\bar{\gamma}, \bar{Y}$ in $(9.3)_{2}$ and $(9.3)_{3}$. Rationalizing this expression and equating to zero the coefficients of the powers of $Q$ in the resulting polynomial which must be identically zero, we obtain the following necessary conditions:

$$
\begin{aligned}
\bar{L} k-L= \pm_{1}(\bar{K} k-K), \quad 2(k \bar{L}+L)^{2} & =2 k(\bar{L}+k L) \\
1 & +k^{2}-(\bar{K} k-K)^{2}= \pm_{2} 2 k
\end{aligned}
$$

Here and henceforth \pm signs which are to be associated carry the same subscript. Putting $(17.2)_{3}$ into $(17.2)_{2}$ yields

$$
\bar{L}+k L= \pm_{2}(L+k \bar{L})
$$

Take $\pm_{2}$ as + . Then $(17.3)$ becomes $(\bar{L}-L)(1-k)=0$, which yields the cases

$$
\begin{array}{llll}
\text { I: } & \pm_{2}=+, & k=1, & \bar{L} \text { arbitrary. } \\
\text { II: } & \pm_{2}=+, & k \neq 1, & \bar{L}=L .
\end{array}
$$

Now take $\pm_{2}$ as - . Then (17.3) becomes $(\bar{L}+L)(k+1)=0$, and we get the case

$$
\text { III: } \quad \pm_{2}=-, k \text { arbitrary, } \bar{L}=-L .
$$

We now explore these three cases systematically. In case I, (17.2) 1 yields $\bar{L}-L= \pm{ }_{1}(\bar{K}-K)$, while $(17.2)_{2}$ yields $\bar{K}=K$. Hence $k=1, \bar{L}=L, \bar{K}=K$.

In case II, $(17.2)_{1}$ yields $L(k-1)= \pm_{1}(\bar{K} k-K)$, while from $(17.2)_{3}$ we have $\bar{K} k-K= \pm(k-1)$. Hence $\bar{L}=L= \pm 1$.

In case III, from $(17.2)_{1}$ follows $-L(1+k)= \pm_{1}(\bar{K} k-K)$, while $(17.2)_{3}$ reduces to $\bar{K} k-K= \pm(k+1)$. Hence again $L= \pm 1$.

The meaning of these necessary conditions will now be investigated.

The conditions $L=\bar{L}, K=\bar{K}$, which arise in case I, may be written 


$$
\frac{1-(2-\bar{\gamma}) \bar{Y}}{|1-\bar{\gamma}|}=\frac{1-(2-\gamma) Y}{|1-\gamma Y|}, \quad \frac{1+\bar{\gamma} \bar{Y}}{|1-\bar{\gamma} \bar{Y}|}=\frac{1+\gamma Y}{|1-\gamma Y|} .
$$

If both or neither of the fluids be weak conductors, (17.4) implies $\gamma=\bar{\gamma}, Y=\bar{Y}$ : the two fluids are alike. If $\langle\gamma, Y\rangle$ be a weak conductor, but $\langle\bar{\gamma}, \bar{Y}\rangle$ not a weak conductor, then (17.4) yields

$$
\bar{Y}=\frac{1}{\gamma}, \quad \bar{\gamma}=\frac{1}{Y} .
$$

It can be verified easily that this necessary condition is also sufficient for the desired correspondence to exist. Moreover, from (17.5) we have $\bar{\gamma} \bar{Y}=(\gamma Y)^{-1}$. Since $\gamma \geqq 1,(17.5)_{1}$ yields $\bar{Y} \leqq 1$, so that $\langle\bar{\gamma}, \bar{Y}\rangle$ is a submoderate conductor. If $\langle\gamma, Y\rangle$ be piezotropic, $\gamma=1$, and hence $\bar{Y}=1$, so that $\langle\bar{\gamma}, \bar{Y}\rangle$ is pseudopiezic. It is this fact which suggests the name "pseudopiezic".

To establish the frequency correspondence, note that since $k=1$ we have $Q=\bar{Q}$, or $\bar{X}(\bar{\gamma} \bar{Y}-1)=X(1-\gamma Y)$. Hence

$$
\bar{X}=\gamma Y X=\frac{1}{\bar{\gamma} \bar{Y}} X
$$

A part of the result we have just established may be put as follows: all absorption and dispersion measures in the weak conductor $\langle\gamma, Y\rangle$ at frequency number $X$ have the same numerical values as the corresponding measures in the submoderate conductor $\left\langle Y^{-1}, \gamma^{-1}\right\rangle$ at the lower frequency number $\gamma Y X$, and conversely.

This result reduces the entire theory of absorption and dispersion in submoderate conductors to that in weak conductors, already discussed in some detail. The fluids $\langle\gamma, Y\rangle$ and $\left\langle Y^{-1}, \gamma^{-1}\right\rangle$ may be called duals of one another. Unfortunately the approximations of $\$ 15$ are not very helpful here, since when $\langle\gamma, Y\rangle$ is a very weak conductor, its dual $\langle\bar{\gamma}, \bar{Y}\rangle$ has a very large value of $\bar{\gamma}$, a case to which no physical fluid corresponds. In $\$ 18$, however, we shall see that a great deal can be learned about moderate conductors from the typical case of the pseudopiezic fluids.

Coming now to cases II and III, we note that $L= \pm 1$ implies $Y=0, Y=1$, or $\gamma=1$. Hence each of the fluids is piezotropic, pseudopiezic, or a non-conductor. While $k$ remains arbitrary, indicating that an infinite number of correspondences between fluids of these types can be set up, we shall not pursue the matter since the explicit formulae for these three types of fluids are so simple.

Summary of the analysis in the preceding pages yields the

Duality theorem. Let it be desired to find two fluids such that the absorption and dispersion curves for the one may be brought into coincidence by uniform changes of scale in magnitude and frequency. Set aside the trivial case when both are alike, and the special cases when both are piezotropic, pseudopiezic, or nonconductors. Then such a correspondence is possible if and only if 
a. One fluid is a weak conductor, $\langle\gamma, Y\rangle$.

b. The other is its dual, the submoderate conductor $\left\langle Y^{-1}, \gamma^{-1}\right\rangle$. The magnitudes of the absorption and dispersion measures for $\langle\gamma, Y\rangle$ at frequency number $X$ equal the magnitudes of the corresponding measures for $\left\langle Y^{-1}, \gamma^{-1}\right\rangle$ at frequency number $\gamma Y X$.

To construct the dual fluid in the $\gamma-Y$ plane, one erects parallels to the axes from $\langle\gamma, Y\rangle$ to the curve $\gamma Y=1$. The point $\langle\bar{\gamma}, \bar{Y}\rangle$ is then the opposite vertex of the rectangle having these segments for two of its sides (Fig. 18.1).

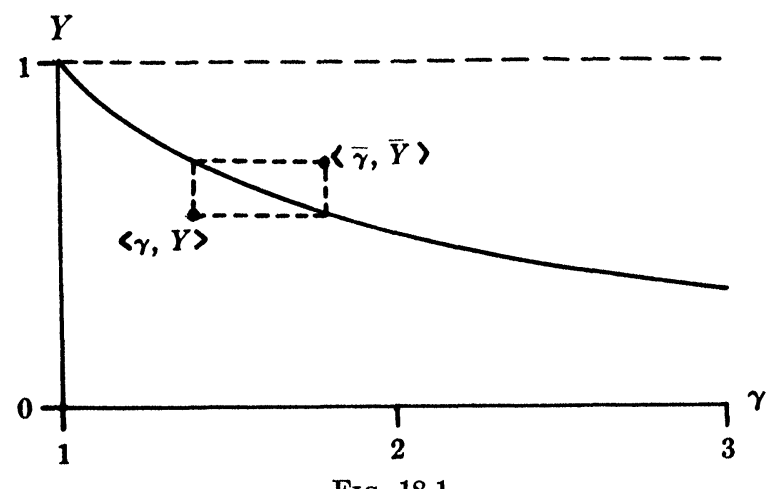

Fig. 18.1

Construction for the duality theorem

18. First approximation for gases : the pseudopiezic fluids. Since in monatomic gases $Y$ is close to 1 (Table 3.1 ), the pseudopiezic fluids $\langle\gamma, 1\rangle$ can serve as a first approximation. An example of a fluid with $Y=1$ is one in which $P=\frac{3}{4}$ and the Stokes relation holds - a case which is well known for the simplification it yields in the shock layer problem. A special case of the duality theorem of $\$ 17$ is the statement that the absorption and dispersion measures for the pseudopiezic fluid $\langle\gamma, 1\rangle$ at frequency number $X$ have the same values as for the piezotropic fluid $\left\langle 1, \gamma^{-1}\right\rangle$ at frequency number $\gamma X$.

Now the results of $\$ 13$ indicate that that for moderate conductors it is only type I waves which will be observed physically. Since type I waves in a piezotropic fluid are unaffected by the value of $Y$, the italicized statement above yields the

First approximation theorem for gases. In the pseudopiezic fluid $\langle\gamma, 1\rangle$ the absorption and dispersion measures at frequency number $X$ are the same as those in a piezotropic fluid (formulae (4.10)) at frequency number $\gamma X$. Consequently the absorption peaks are given by

$$
\begin{gathered}
X^{*}=\frac{1}{\gamma}<X \nvdash=\frac{\sqrt{3}}{\gamma}, \quad a^{*}=\frac{1}{2} \pi<A_{0} \downarrow=\frac{\pi}{\sqrt{2}}, \\
\frac{\lambda}{\mu}=-2+\frac{1}{\gamma S^{*}}=-2+\frac{\sqrt{3}}{\gamma S^{\natural}} .
\end{gathered}
$$

Since for gases $\gamma \leqq \frac{5}{3}$, these results indicate that the whole absorption and 
dispersion behavior of gases is very much like that in a piezotropic fluid, not only qualitatively, but also in order of magnitude. Resonance occurs at a somewhat lower frequency than when $\gamma=1$, but the height of the resonant peaks is the same.

19. Second approximation for gases. We now expand the general formulae at $\S 10$ in powers of $Y-1$. After some calculation we obtain the following results, with error $O\left([(\gamma-1)(Y-1)]^{2}\right)$ :

$$
\begin{aligned}
& \frac{a}{a_{\psi}}=1+\frac{\gamma-1}{\gamma}(Y-1) \frac{1-\gamma(\gamma-1) X^{2}}{1+(\gamma-1)^{2} X^{2}} \\
& \frac{r}{r_{\psi}}=1+(\gamma-1)(Y-1) \frac{X^{2}}{1+(\gamma-1)^{2} X^{2}}\left[\gamma-1+\frac{\gamma}{1+\sqrt{1+\gamma^{2} X^{2}}}\right] \\
& \frac{A}{A_{\psi}}=1+\frac{\gamma-1}{\gamma}(Y-1) \frac{\sqrt{1+\gamma^{2} X^{2}}}{1+(\gamma-1)^{2} X^{2}} \\
& \frac{A_{0}}{A_{0 \psi}}=1+\frac{\frac{1}{2} \frac{\gamma-1}{\gamma}(Y-1)}{1+(\gamma-1)^{2} X^{2}}\left[1+\sqrt{1+\gamma^{2} X^{2}}-\gamma(\gamma-1) X^{2}\right]
\end{aligned}
$$

The subscript $\psi$ denotes the measures for a pseudopiezic fluid, given explicitly by the theorem of $\S 18$. For the three fluids $\langle 1.25, .8\rangle,\left\langle\frac{5}{3}, .8\right\rangle,\left\langle\frac{5}{3}, 1.05\right\rangle$, the greatest error in the results given by the foregoing expressions in the range $0 \leqq$ $X \leqq 5$ is $7 \%$, the absorption formulae being accurate in fact to within $2 \%$ for the two latter fluids.

Examination of (19.1)-(19.4) leads to the

Approximation theorem for moderate conductors. Let the absorption and dispersion measures for moderate conductors be compared up to terms

$$
O\left([(\gamma-1)(Y-1)]^{2}\right)
$$

with those for pseudopiezic fluids. Then at all frequencies the dispersion and the true absorption coefficient $A$ are less (greater) in the submoderate (supermoderate) conductor $\langle\gamma, Y\rangle$ than in the pseudopiezic fluid $\langle\gamma, 1\rangle$. The ratios $Q / Q_{\psi}$ and $A_{0} / A_{0 \psi}$, however, are less than (greater than) 1 at low frequencies in submoderate (supermoderate) conductors, greater than (less than) 1 at high frequencies.

For the resonant peak in $Q$ we have

$$
\begin{aligned}
X^{*} \approx \frac{1}{\gamma}-2(Y-1) \frac{(\gamma-1)^{2}(2 \gamma-1)}{\left[(\gamma-1)^{2}+\gamma^{2}\right]^{2}} & \\
A^{*} & \approx \frac{1}{2} \pi\left[1+\frac{(\gamma-1)(Y-1)}{(\gamma-1)^{2}+\gamma^{2}}\right] .
\end{aligned}
$$

Consequently $X^{*}>1 / \gamma$ if $Y<1$, while $X^{*}<1 / \gamma$ if $Y>1$. Also $A^{*}<\frac{1}{2} \pi$ if $Y$ 
$<1$, while $A^{*}>\frac{1}{2} \pi$ if $Y>1$. Instead of formalizing what we have just proved as a theorem, we may combine it with the information gained in $\$ 15$ as well as from numerical examples to formulate the following

Conjecture regarding visco-thermal resonance in weak and moderate conductors. Let $\gamma$ be fixed at any value greater than 1 , and let $Y$ increase steadily from 0 to $2-\gamma$ if $\gamma<2$, to $\infty$ if $\gamma \geqq 2$. Then the resonant frequency number $X^{*}$ for the measure $Q$ decreases steadily from the value $X^{*}=1$ when $Y=0$, passing through $X^{*}=\gamma^{-1}$ when $Y=1$. The height $a^{*}$ of the resonant peak at first decreases from the value $\frac{1}{2} \pi$ when $Y=0$, experiences a minimum, rises again to the value $\frac{1}{2} \pi$ when $Y=1$, and continues to increase thereafter.

On less firm ground but of greater physical interest is the conjecture that in the above statement, Q may be replaced by $A_{0}$, with appropriate numerical changes ( $\sqrt{3} / \gamma$ for $1 / \gamma, \pi / \sqrt{2}$ for $\frac{1}{2} \pi$, etc.). The basis for this more extensive guess is that examples seem to indicate the measure $A_{0}$ to have a qualitative behavior quite similar to that of $Q$ in any given fluid, except that its resonant peak is always much flatter.

We may study also the effect of change of $\gamma$ when $Y$ is fixed, provided we remain in the region of moderate conductors. As $\gamma$ increases from 1 to $\infty$ the coefficient of $(Y-1) / \gamma$ in $(19.5)_{1}$ decreases steadily from 0 to -1 . Thus increase of $\gamma$ increases (decreases) $\gamma X^{*}$ for submoderate (supermoderate) conductors. The coefficient of $Y-1$ in $(19.5)_{2}$, however, increases from the value 0 when $\gamma=1$ to the value $(2+2 \sqrt{2})^{-\frac{1}{2}}$ when $\gamma=1+2^{-\frac{1}{2}}$, falling off thereafter to 0 . Hence the height of the resonant peak, taken as a function of $\gamma$ when $Y$ is fixed, for a submoderate (supermoderate) conductor decreases (increases) from the value $\frac{1}{2} \pi$ at $\gamma=1$ to a minimum (maximum) when $\gamma=1+2^{-\frac{1}{2}}$, waxing (waning) thereafter to the value $\frac{1}{2} \pi$ as $\gamma \rightarrow \infty$.

20. The singular fluids. For the singular fluids $B$, defined by the condition $\gamma Y=1$, the exact solution of $\$ 10$ remains valid. The calculations are relatively simple, since (10.1) reduces to

$$
E=1, \quad F=4 X(1-Y) .
$$

It is easy to see that the power series expansion method of $\$ 14$ is valid if and only if $X<[4(1-Y)]^{-1}$, and that the resulting measures pertain to type I waves. In the sense of the duality theorem of $\$ 17$, the singular fluids $B$ are self-dual.

In some liquefied gases $\gamma \approx 2$ and $Y \approx \frac{1}{2}$ (see Table 3.1). Thus the singular fluids $B$ may serve as a first approximation for them.

No physical fluids appear to be approximated by the singular fluids A, defined by the condition $Y(2-\gamma)=1$. For completeness, however, we shall determine the appropriate measures. Since in effect we are assigning va. to a complex function at points on a cut, there must be considerable arbitrariness in our procedure. We shall define two wave types in the only way which can 
permit extension of the first limit theorem of $\$ 9$ to the case when the set of points $\left\langle\gamma_{P}, Y_{P}\right\rangle$ includes or consists wholly in singular fluids $\mathrm{A}$ and the limit fluid is $\langle 1,1\rangle$. (Of course the analyticity theorem cannot be so extended.)

It is easiest to proceed directly from the Kirchhoff-Langevin equation (4.16), which in the present special case can be written

$$
\begin{aligned}
\left\{2\left(\frac{2 \pi}{\sigma}\right)^{2}+1+\left(1-Z^{2}\right)^{\frac{1}{2}}\right. & \left.+\frac{i Z}{\gamma-1}\right\} \\
\cdot & \left\{2\left(\frac{2 \pi}{\sigma}\right)^{2}+1-\left(1-Z^{2}\right)^{\frac{1}{2}}+\frac{i Z}{\gamma-1}\right\}=0
\end{aligned}
$$

where $Z \equiv(\gamma-1) X /\left(1-\frac{1}{2} \gamma\right)$. There are now two frequency ranges which must be distinguished: ${ }^{1} Z<1$ and $Z \geqq 1$, or

$$
\begin{array}{ll}
\alpha: & X<\frac{1-\frac{1}{2} \gamma}{\gamma-1}, \\
\beta: & X \geqq \frac{1-\frac{1}{2} \gamma}{\gamma-1} .
\end{array}
$$

Since as $\gamma \rightarrow 1+$ the range $\alpha$ increases so as to become $0 \leqq X<\infty$, all that is required is to find a pair of determinations of $G$ and $H$, continuous functions of $X$ for $0 \leqq X<\infty$, which as $\gamma \rightarrow 1+$ in range $\alpha$ will approach, respectively, the values $G=1, H=X$ and $G=0, H=X Y=X$, appropriate to the piezotropic case. From (20.2) it is easy to see that

$$
\begin{aligned}
G & = \begin{cases}\frac{1}{2}\left(1 \pm \sqrt{\left.1-Z^{2}\right)}\right. & \text { in range } \alpha, \\
\frac{1}{2} & \text { in range } \beta,\end{cases} \\
H & = \begin{cases}\frac{1}{2} Z /(\gamma-1) & \text { in range } \alpha, \\
\frac{1}{2}\left[Z /(\gamma-1) \pm \sqrt{Z^{2}-1}\right] & \text { in range } \beta,\end{cases}
\end{aligned}
$$

where, by definition, the upper signs yield type I waves, the lower, type II waves. All absorption and dispersion measures are continuous functions of $X$, but their derivatives are only piecewise continuous.

21. Tables. The following tables were computed from the exact formulae of $\$ 10$ by Mr. Harrison Hancock of the Applied Mathematics Branch, Mechanics Division, Naval Research Laboratory. Their error is at most \pm 1 in the third significant figure.

If, for a given value of $Y$ (or $\gamma$ ) one should desire solutions for fluids with $\gamma$

1 Such a division of range was not required in the general case treated in $\$ 10$, since the assumption $Y(2-\gamma) \neq 1$ made there implies $F \neq 0$ if $X \neq 0$, whence follows $E^{2}+F^{2} \neq 0$ for all $X$. 
(or $Y$ ) smaller than any for which tables are provided, then the piezotropic fluid with the same value of $Y$ (or the non-conductor with the same value of $\gamma$ ) furnishes a sufficiently good approximation, or may be used as a basis for interpolation.

Figs. 21.1-21.4 present the results in graphical form for typical examples of weak, submoderate, supermoderate, and strong conductors.

As a trial, the case $\gamma=5, Y=50$ was computed roughly. The results, which will not be presented in detail, were qualitatively similar to those for snualler values of $\gamma$ and $Y$.

There is reason to believe that the analysis and tables in this memoir give a full solution to the problem which we set out to solve, valid over the range of parameter values which can be of physical interest. 
Group 0. The piezotropic fluids $\langle 1, Y\rangle$

\begin{tabular}{|c|c|c|c|c|c|c|c|c|c|c|}
\hline$x$ & $r_{p}$ & $a_{p}$ & $A_{0 p}$ & $A_{p}$ & $r_{p}^{\prime} / Y$ & $Y Q_{p}^{\prime}$ & $\sqrt{\bar{Y}} A_{0 p}^{\prime}$ & $A_{p}^{\prime}$ & $\Omega_{p} / Y$ & $Y Q_{p}$ \\
\hline 0 & 1 & 0 & 0 & 0 & 0 & $\infty$ & $\infty$ & 6.28 & 0 & $\infty$ \\
\hline .1 & 1.01 & .311 & .312 & .313 & .2 & 31.4 & 14.0 & 6.28 & .199 & 2030 \\
\hline .3 & 1.07 & .865 & .893 & .922 & .6 & 10.5 & 8.11 & 6.28 & .562 & 82.5 \\
\hline .5 & 1.18 & 1.26 & 1.37 & 1.48 & 1 & 6.28 & 6.28 & 6.28 & .847 & 21.2 \\
\hline .7 & 1.34 & 1.48 & 1.71 & 1.98 & 1.4 & 4.49 & 5.31 & 6.28 & 1.04 & 9.65 \\
\hline 1. & 1.66 & 1.57 & 2.02 & 2.60 & 2 & 3.14 & 4.44 & 6.28 & 1.21 & 4.83 \\
\hline 1.5 & 2.32 & 1.45 & 2.21 & 3.36 & 3 & 2.09 & 3.63 & 6.28 & 1.29 & 2.70 \\
\hline 2. & 3.09 & 1.26 & 2.21 & 3.88 & 4 & 1.57 & 3.14 & 6.28 & 1.29 & 2.02 \\
\hline 2.5 & 3.93 & 1.08 & 2.15 & 4.25 & 5 & 1.26 & 2.81 & 6.28 & 1.27 & 1.71 \\
\hline 3. & 4.81 & .942 & 2.07 & 4.53 & 6 & 1.05 & 2.57 & 6.28 & 1.25 & 1.54 \\
\hline 3.5 & 5.71 & .830 & 1.98 & 4.74 & 7 & .898 & 2.37 & 6.28 & 1.23 & 1.43 \\
\hline 4. & 6.64 & .739 & 1.90 & 4.91 & 8 & .785 & 2.22 & 6.28 & 1.21 & 1.36 \\
\hline 4.5 & 7.58 & .665 & 1.83 & 5.04 & 9 & .698 & 2.09 & 6.28 & 1.19 & 1.31 \\
\hline 5. & 8.53 & .604 & 1.76 & 5.15 & 10 & .628 & 1.99 & 6.28 & 1.17 & 1.27 \\
\hline
\end{tabular}

Group I. Weak and moderate conductors

$\langle 1.10, .5\rangle$

\begin{tabular}{|c|c|c|c|c|c|c|c|c|c|c|}
\hline$x$ & $r$ & $a$ & $\Delta_{0}$ & $A$ & $r^{\prime}$ & $a^{\prime}$ & $A_{0}^{\prime}$ & $A^{\prime}$ & $\mathbf{\Omega}$ & $Q$ \\
\hline 0 & 1 & 0 & 0 & 0 & 0 & $\infty$ & $\infty$ & 6.28 & 0 & $\infty$ \\
\hline .1 & 1.01 & .326 & .328 & .329 & .100 & 62.8 & 19.9 & 6.31 & .0996 & 3690 . \\
\hline .3 & 1.08 & .896 & .929 & .963 & .305 & 20.9 & 11.5 & 6.38 & .284 & 154. \\
\hline .5 & 1.20 & 1.28 & 1.40 & 1.54 & .515 & 12.5 & 8.96 & 6.43 & .429 & 40.8 \\
\hline .7 & 1.38 & 1.48 & 1.73 & 2.03 & .730 & 8.87 & 7.58 & 6.48 & .530 & 19.1 \\
\hline 1. & 1.71 & 1.54 & 2.02 & 2.64 & 1.06 & 6.15 & 6.33 & 6.52 & .619 & 9.85 \\
\hline 1.5 & 2.40 & 1.40 & 2.17 & 3.36 & 1.63 & 4.04 & 5.15 & 6.57 & .679 & 5.62 \\
\hline 2. & 3.17 & 1.21 & 2.16 & 3.85 & 2.20 & 2.99 & 4.43 & 6.58 & .694 & 4.21 \\
\hline 2.5 & 4.00 & 1.05 & 2.10 & 4.20 & 2.77 & 2.37 & 3.94 & 6.57 & .693 & 3.53 \\
\hline 3. & 4.86 & .917 & 2.02 & 4.46 & 3.34 & 1.96 & 3.59 & 6.56 & .687 & 3.15 \\
\hline 3.5 & 5.75 & .811 & 1.94 & 4.66 & 3.91 & 1.67 & 3.31 & 6.54 & .680 & 2.89 \\
\hline 4. & 6.66 & .724 & 1.87 & 4.83 & 4.48 & 1.46 & 3.08 & 6.52 & .672 & 2.72 \\
\hline 4.5 & 7.58 & .654 & 1.80 & 4.96 & 5.04 & 1.29 & 2.90 & 6.51 & .665 & 2.59 \\
\hline 5. & 8.52 & .595 & 1.74 & 5.07 & 5.60 & 1.16 & 2.74 & 6.50 & .658 & 2.49 \\
\hline \multicolumn{11}{|c|}{$\langle 1.10, .8\rangle$} \\
\hline 0 & 1 & 0 & 0 & 0 & 0 & $\infty$ & $\infty$ & 6.28 & 0 & $\infty$ \\
\hline .1 & 1.01 & .335 & .337 & .338 & .160 & 39.3 & 15.7 & 6.30 & .159 & 2180 . \\
\hline .3 & 1.08 & .919 & .954 & .991 & .483 & 13.1 & 9.10 & 6.32 & .448 & 90.9 \\
\hline .5 & 1.21 & 1.30 & 1.44 & 1.58 & .809 & 7.84 & 7.06 & 6.34 & .668 & 24.1 \\
\hline .7 & 1.40 & 1.50 & 1.77 & 2.09 & 1.14 & 5.60 & 5.97 & 6.37 & .814 & 11.4 \\
\hline 1. & 1.76 & 1.54 & 2.05 & 2.71 & 1.64 & 3.91 & 5.00 & 6.40 & .933 & 5.97 \\
\hline 1.5 & 2.48 & 1.38 & 2.18 & 3.44 & 2.49 & 2.59 & 4.09 & 6.44 & 1.00 & 3.51 \\
\hline 2. & 3.30 & 1.19 & 2.16 & 3.92 & 3.35 & 1.93 & 3.54 & 6.47 & 1.02 & 2.69 \\
\hline 2.5 & 4.17 & 1.02 & 2.08 & 4.25 & 4.23 & 1.54 & 3.16 & 6.50 & 1.01 & 2.31 \\
\hline 3. & 5.07 & .885 & 1.99 & 4.49 & 5.11 & 1.27 & 2.88 & 6.51 & 1.01 & 2.09 \\
\hline 3.5 & 5.99 & .780 & 1.91 & 4.68 & 6.00 & 1.09 & 2.66 & 6.52 & 1.00 & 1.94 \\
\hline 4. & 6.92 & .697 & 1.83 & 4.82 & 6.89 & .947 & 2.49 & 6.53 & .996 & 1.84 \\
\hline 4.5 & 7.86 & .629 & 1.76 & 4.94 & 7.79 & .838 & 2.34 & 6.53 & .991 & 1.76 \\
\hline 5. & 8.80 & .573 & 1.70 & 5.04 & 8.69 & .752 & 2.22 & 6.53 & .987 & 1.70 \\
\hline
\end{tabular}


$\langle 1.25, .25\rangle$

\begin{tabular}{|c|c|c|c|c|c|c|c|c|c|c|}
\hline$x$ & $r$ & a & $A_{0}$ & $\boldsymbol{A}$ & $r^{\prime}$ & $a^{\prime}$ & $A_{0}^{\prime}$ & $A^{\prime}$ & $\Omega$ & $Q$ \\
\hline 0 & 1 & 0 & 0 & 0 & 0 & $\infty$ & $\infty$ & 6.28 & 0 & $\infty$ \\
\hline .1 & 1.01 & .330 & .331 & .333 & .0510 & 125. & 28.3 & 6.40 & .0506 & 7310 . \\
\hline .3 & 1.08 & .900 & .934 & .970 & .160 & 41.2 & 16.5 & 6.62 & .149 & 313. \\
\hline .5 & 1.21 & 1.28 & 1.40 & 1.54 & .280 & 24.2 & 12.8 & 6.79 & .232 & 83.6 \\
\hline .7 & 1.38 & 1.47 & 1.72 & 2.03 & .409 & 16.9 & 10.8 & 6.90 & .295 & 39.1 \\
\hline 1. & 1.72 & 1.53 & 2.00 & 2.62 & .611 & 11.4 & 8.93 & 6.98 & .356 & 19.9 \\
\hline 1.5 & 2.39 & 1.40 & 2.16 & 3.33 & .955 & 7.31 & 7.14 & 6.98 & .400 & 11.0 \\
\hline 2. & 3.15 & 1.21 & 2.15 & 3.82 & 1.30 & 5.34 & 6.08 & 6.93 & .412 & 7.97 \\
\hline 2.5 & 3.97 & 1.05 & 2.10 & 4.18 & 1.63 & 4.20 & 5.37 & 6.86 & .411 & 6.55 \\
\hline 3. & 4.83 & .921 & 2.02 & 4.45 & 1.96 & 3.46 & 4.85 & 6.80 & .406 & 5.75 \\
\hline 3.5 & 5.72 & .814 & 1.95 & 4.66 & 2.29 & 2.95 & 4.46 & 6.75 & .400 & 5.25 \\
\hline 4. & 6.63 & .728 & 1.87 & 4.82 & 2.61 & 2.56 & 4.14 & 6.70 & .394 & 4.89 \\
\hline 4.5 & 7.56 & .656 & 1.80 & 4.96 & 2.93 & 2.27 & 3.89 & 6.66 & .388 & 4.65 \\
\hline 5. & 8.49 & .597 & 1.74 & 5.07 & 3.25 & 2.04 & 3.68 & 6.63 & .383 & 4.46 \\
\hline
\end{tabular}

$\langle 1.25, .5\rangle$

\begin{tabular}{|c|c|c|c|c|c|c|c|c|c|c|}
\hline 0 & 1 & 0 & 0 & 0 & 0 & $\infty$ & $\infty$ & 6.28 & 0 & $\infty$ \\
\hline .1 & 1.01 & .348 & .350 & .352 & .101 & 62.8 & 19.9 & 6.36 & .100 & 3250. \\
\hline .3 & 1.09 & .941 & .982 & 1.02 & .313 & 20.8 & 11.6 & 6.51 & .288 & 140. \\
\hline .5 & 1.23 & 1.31 & 1.45 & 1.62 & .539 & 12.3 & 9.05 & 6.64 & .437 & 38.7 \\
\hline .7 & 1.43 & 1.48 & 1.76 & 2.11 & .778 & 8.67 & 7.64 & 6.74 & .544 & 18.7 \\
\hline 1. & 1.80 & 1.50 & 2.01 & 2.69 & 1.15 & 5.93 & 6.37 & 6.84 & .643 & 10.0 \\
\hline 1.5 & 2.51 & 1.34 & 2.13 & 3.37 & 1.81 & 3.82 & 5.14 & 6.92 & .721 & 5.86 \\
\hline 2. & 3.30 & 1.16 & 2.10 & 3.82 & 2.48 & 2.80 & 4.40 & 6.94 & .753 & 4.38 \\
\hline 2.5 & 4.13 & 1.00 & 2.04 & 4.14 & 3.15 & 2.19 & 3.90 & 6.92 & .764 & 3.66 \\
\hline 3. & 4.98 & .879 & 1.96 & 4.38 & 3.82 & 1.80 & 3.52 & 6.89 & .768 & 3.23 \\
\hline 3.5 & 5.86 & .779 & 1.89 & 4.57 & 4.50 & 1.53 & 3.24 & 6.86 & .767 & 2.94 \\
\hline 4. & 6.75 & .699 & 1.82 & 4.72 & 5.16 & 1.32 & 3.01 & 6.84 & .764 & 2.74 \\
\hline 4.5 & 7.66 & .634 & 1.75 & 4.85 & 5.82 & 1.17 & 2.82 & 6.81 & .760 & 2.59 \\
\hline 5. & 8.57 & .578 & 1.69 & 4.96 & 6.48 & 1.05 & 2.66 & 6.78 & .756 & 2.47 \\
\hline \multicolumn{11}{|c|}{$\langle 1.25, .8\rangle$} \\
\hline 0 & 1 & 0 & 0 & 0 & 0 & $\infty$ & $\infty$ & 6.28 & 0 & $\infty$ \\
\hline .1 & 1.01 & .372 & .374 & .376 & .161 & 39.3 & 15.7 & 6.32 & .159 & 1770. \\
\hline .3 & 1.10 & .994 & 1.04 & 1.09 & .488 & 13.1 & 9.13 & 6.38 & . .444 & 76.9 \\
\hline .5 & 1.26 & 1.36 & 1.53 & 1.72 & .823 & 7.81 & 7.09 & 6.43 & .652 & 21.4 \\
\hline .7 & 1.48 & 1.51 & 1.84 & 2.25 & 1.17 & 5.56 & 6.00 & 6.48 & .786 & 10.6 \\
\hline 1. & 1.90 & 1.50 & 2.07 & 2.86 & 1.70 & 3.86 & 5.03 & 6.55 & .893 & 5.88 \\
\hline 1.5 & 2.72 & 1.30 & 2.15 & 3.55 & 2.61 & 2.54 & 4.10 & 6.63 & .961 & 3.63 \\
\hline 2. & 3.62 & 1.10 & 2.09 & 3.98 & 3.56 & 1.88 & 3.54 & 6.68 & .983 & 2.86 \\
\hline 2.5 & 4.55 & .941 & 2.01 & 4.28 & 4.51 & 1.48 & 3.16 & 6.70 & .992 & 2.47 \\
\hline 3. & 5.51 & .817 & 1.92 & 4.50 & 5.48 & 1.22 & 2.87 & 6.72 & .995 & 2.24 \\
\hline 3.5 & 6.48 & .720 & 1.83 & 4.67 & 6.46 & 1.04 & 2.65 & 6.72 & .997 & 2.08 \\
\hline 4. & 7.46 & .644 & 1.76 & 4.80 & 7.44 & .905 & 2.47 & 6.73 & .998 & 1.97 \\
\hline 4.5 & 8.44 & .582 & 1.69 & 4.91 & 8.43 & .799 & 2.32 & 6.73 & .999 & 1.88 \\
\hline 5. & 9.42 & .530 & 1.63 & 5.00 & 9.42 & .715 & 2.19 & 6.73 & .999 & 1.81 \\
\hline
\end{tabular}


$\langle 1.40, .25\rangle$

\begin{tabular}{|c|c|c|c|c|c|c|c|c|c|c|}
\hline$X$ & $r$ & $Q$ & $A_{0}$ & $A$ & $r^{\prime}$ & $\dot{Q}^{\prime}$ & $A_{0}^{\prime}$ & $A^{\prime}$ & $\mathbf{\Omega}$ & $Q$ \\
\hline 0 & 1 & 0 & 0 & 0 & 0 & $\infty$ & $\infty$ & 6.28 & 0 & $\infty$ \\
\hline .1 & 1.01 & .340 & .342 & .344 & .0517 & 125. & 28.5 & 6.47 & .0512 & 6920. \\
\hline .3 & 1.08 & .921 & .960 & 1.00 & .167 & 40.7 & 16.7 & 6.82 & .154 & 301. \\
\hline .5 & 1.22 & 1.29 & 1.42 & 1.58 & .301 & 23.6 & 12.9 & 7.09 & .246 & 82.3 \\
\hline .7 & 1.41 & 1.46 & 1.73 & 2.06 & .448 & 16.2 & 10.8 & 7.24 & .317 & 38.9 \\
\hline 1. & 1.76 & 1.50 & 1.99 & 2.64 & .682 & 10.8 & 8.89 & 7.34 & .389 & 19.9 \\
\hline 1.5 & 2.43 & 1.37 & 2.13 & 3.32 & 1.08 & 6.77 & 7.04 & 7.33 & .445 & 10.9 \\
\hline 2. & 3.19 & 1.19 & 2.12 & 3.79 & 1.48 & 4.90 & 5.95 & 7.24 & .463 & 7.87 \\
\hline 2.5 & 4.01 & 1.03 & 2.07 & 4.14 & 1.87 & 3.83 & 5.23 & 7.14 & .465 & 6.39 \\
\hline 3. & 4.86 & .906 & 2.00 & 4.40 & 2.24 & 3.14 & 4.71 & 7.05 & .462 & 5.56 \\
\hline 3.5 & 5.74 & .803 & 1.92 & 4.61 & 2.62 & 2.67 & 4.31 & 6.98 & .456 & 5.03 \\
\hline 4. & 6.64 & .719 & 1.85 & 4.78 & 2.99 & 2.31 & 4.00 & 6.91 & .450 & 4.67 \\
\hline 4.5 & 7.55 & .650 & 1.79 & 4.91 & 3.35 & 2.04 & 3.74 & 6.85 & .444 & 4.38 \\
\hline 5. & 8.48 & .593 & 1.73 & 5.03 & 3.71 & 1.83 & 3.53 & 6.80 & .438 & 4.19 \\
\hline \multicolumn{11}{|c|}{$\langle 1.40, .5\rangle$} \\
\hline 0 & 1 & 0 & 0 & 0 & 0 & $\infty$ & $\infty$ & 6.28 & 0 & $\infty$ \\
\hline .1 & 1.01 & .371 & .373 & .375 & .102 & 62.7 & 20.1 & 6.41 & .101 & 2900 . \\
\hline .3 & 1.10 & .982 & 1.03 & 1.08 & .322 & 20.6 & 11.7 & 6.65 & .292 & 129 \\
\hline .5 & 1.27 & 1.33 & 1.50 & 1.69 & .565 & 12.1 & 9.11 & 6.85 & .446 & 36.8 \\
\hline .7 & 1.49 & 1.47 & 1.79 & 2.19 & .827 & 8.45 & 7.68 & 6.99 & .557 & 18.4 \\
\hline 1. & 1.88 & 1.47 & 2.01 & 2.75 & 1.25 & 5.71 & 6.37 & 7.2 & .664 & 10.1 \\
\hline 1.5 & 2.63 & 1.29 & 2.09 & 3.39 & 1.98 & 3.63 & 5.12 & 7.21 & .755 & 5.98 \\
\hline 2. & 3.44 & 1.11 & 2.05 & 3.81 & 2.74 & 2.63 & 4.36 & 7.22 & .799 & 4.50 \\
\hline 2.5 & 4.28 & .960 & 1.99 & 4.11 & 3.51 & 2.05 & 3.84 & 7.19 & .820 & 3.74 \\
\hline 3. & 5.14 & .842 & 1.91 & 4.33 & 4.27 & 1.68 & 3.46 & 7.16 & .830 & 3.29 \\
\hline 3.5 & 6.02 & .748 & 1.84 & 4.51 & 5.03 & 1.42 & 3.18 & 7.12 & .835 & 2.99 \\
\hline 4. & 6.91 & .673 & 1.77 & 4.65 & 5.79 & 1.22 & 2.94 & 7.08 & .838 & 2.77 \\
\hline 4.5 & 7.81 & .611 & 1.71 & 4.77 & 6.54 & 1.08 & 2.76 & 7.05 & .838 & 2.61 \\
\hline 5. & 8.72 & .559 & 1.65 & 4.87 & 7.30 & .961 & 2.60 & 7.01 & .837 & 2.47 \\
\hline \multicolumn{11}{|c|}{$\langle 1.40, .8\rangle$} \\
\hline 0 & 1 & 0 & 0 & 0 & 0 & $\infty$ & $\infty$ & 6.28 & 0 & $\infty$ \\
\hline .1 & 1.01 & .407 & .410 & .413 & .161 & 39.3 & 15.8 & 6.33 & .159 & 1490 \\
\hline .3 & 1.12 & 1.06 & 1.12 & 1.19 & .493 & 13.0 & 9.16 & 6.43 & .441 & 66.3 \\
\hline .5 & 1.31 & 1.41 & 1.62 & 1.85 & .839 & 7.77 & 7.12 & 6.52 & .638 & 19.4 \\
\hline .7 & 1.57 & 1.52 & 1.91 & 2.39 & 1.20 & 5.50 & 6.02 & 6.59 & .760 & 9.97 \\
\hline 1. & 2.05 & 1.46 & 2.10 & 3.00 & 1.76 & 3.80 & 5.04 & 6.67 & .858 & 5.78 \\
\hline 1.5 & 2.95 & 1.24 & 2.13 & 3.65 & 2.73 & 2.48 & 4.09 & 6.76 & .923 & 3.70 \\
\hline 2. & 3.93 & 1.03 & 2.05 & 4.07 & 3.73 & 1.82 & 3.52 & 6.80 & .948 & 2.95 \\
\hline 2.5 & 4.95 & .880 & 1.96 & 4.35 & 4.74 & 1.44 & 3.13 & 6.82 & .958 & 2.56 \\
\hline 3. & 5.98 & .762 & 1.86 & 4.56 & 5.76 & 1.18 & 2.84 & 6.82 & .964 & 2.33 \\
\hline 3.5 & 7.02 & .671 & 1.78 & 4.72 & 6.79 & 1.00 & 2.62 & 6.82 & .967 & 2.16 \\
\hline 4. & 8.07 & .600 & 1.70 & 4.84 & 7.82 & .871 & 2.44 & 6.81 & .969 & 2.04 \\
\hline 4.5 & 9.13 & .542 & 1.64 & 4.95 & 8.86 & .769 & 2.29 & 6.81 & .970 & 1.95 \\
\hline 5. & 10.2 & .494 & 1.58 & 5.03 & 9.89 & .687 & 2.16 & 6.80 & .970 & 1.88 \\
\hline
\end{tabular}




\begin{tabular}{|c|c|c|c|c|c|c|c|c|c|c|}
\hline \multicolumn{11}{|c|}{$\left\langle\frac{5}{3}, .25\right\rangle$} \\
\hline$x$ & $r$ & $a$ & $A_{0}$ & $A$ & $r^{\prime}$ & $a^{\prime}$ & $A_{0}^{\prime}$ & $A^{\prime}$ & $\Omega$ & $Q$ \\
\hline 0 & 1 & 0 & 0 & 0 & 0 & $\infty$ & $\infty$ & 6.28 & 0 & $\infty$ \\
\hline .1 & 1.01 & .360 & .362 & .364 & .0529 & 125. & 28.7 & 6.60 & .0523 & 6280. \\
\hline .3 & 1.10 & .956 & 1.00 & 1.05 & .181 & 39.7 & 16.9 & 7.18 & .165 & 284 \\
\hline .5 & 1.25 & 1.30 & 1.46 & 1.63 & .340 & 22.3 & 13.0 & 7.58 & .271 & 79.9 \\
\hline .7 & 1.46 & 1.45 & 1.75 & 2.11 & .521 & 15.0 & 10.8 & 7.79 & .357 & 38.0 \\
\hline 1. & 1.82 & 1.47 & 1.98 & 2.67 & .814 & 9.71 & 8.76 & 7.90 & .447 & 19.6 \\
\hline 1.5 & 2.52 & 1.32 & 2.09 & 3.31 & 1.31 & 5.98 & 6.85 & 7.84 & .521 & 10.7 \\
\hline 2. & 3.28 & 1.14 & 2.07 & 3.75 & 1.80 & 4.27 & 5.73 & 7.70 & .550 & 7.65 \\
\hline 2.5 & 4.09 & .998 & 2.02 & 4.08 & 2.28 & 3.32 & 5.01 & 7.56 & .558 & 6.16 \\
\hline 3. & 4.93 & .879 & 1.95 & 4.33 & 2.75 & 2.70 & 4.48 & 7.43 & .558 & 5.29 \\
\hline 3.5 & 5.79 & .782 & 1.88 & 4.53 & 3.20 & 2.29 & 4.09 & 7.32 & .553 & 4.72 \\
\hline 4. & 6.68 & .703 & 1.82 & 4.69 & 3.65 & 1.98 & 3.78 & 7.23 & .547 & 4.34 \\
\hline 4.5 & 7.58 & .637 & 1.75 & 4.83 & 4.10 & 1.74 & 3.53 & 7.15 & .541 & 4.06 \\
\hline 5. & 8.49 & .582 & 1.70 & 4.94 & 4.54 & 1.56 & 3.32 & 7.08 & .534 & 3.84 \\
\hline \multicolumn{11}{|c|}{$\left\langle\frac{5}{3}, .5\right\rangle$} \\
\hline 0 & 1 & 0 & 0 & 0 & 0 & $\infty$ & $\infty$ & 6.28 & 0 & $\infty$ \\
\hline .1 & 1.02 & .410 & .412 & .416 & .104 & 62.6 & 20.2 & 6.50 & .102 & 2390 . \\
\hline .3 & 1.13 & 1.05 & 1.12 & 1.19 & .339 & 20.3 & 11.8 & 6.89 & .300 & 112 \\
\hline .5 & 1.33 & 1.37 & 1.58 & 1.82 & .612 & 11.7 & 9.17 & 7.17 & .461 & 33.8 \\
\hline .7 & 1.58 & 1.46 & 1.84 & 2.31 & .916 & 8.03 & 7.69 & 7.36 & .580 & 17.5 \\
\hline 1. & 2.02 & 1.41 & 2.01 & 2.86 & 1.41 & 5.33 & 6.33 & 7.51 & .696 & 9.94 \\
\hline 1.5 & 2.84 & 1.22 & 2.05 & 3.45 & 2.27 & 3.34 & 5.03 & 7.58 & .801 & 6.03 \\
\hline 2. & 3.70 & 1.04 & 1.99 & 3.83 & 3.16 & 2.40 & 4.26 & 7.57 & .853 & 4.58 \\
\hline 2.5 & 4.59 & .893 & 1.91 & 4.10 & 4.05 & 1.86 & 3.74 & 7.52 & .882 & 3.82 \\
\hline 3. & 5.49 & .784 & 1.84 & 4.30 & 4.94 & 1.51 & 3.36 & 7.48 & .901 & 3.36 \\
\hline 3.5 & 6.40 & .697 & 1.76 & 4.46 & 5.83 & 1.27 & 3.08 & 7.43 & .912 & 3.04 \\
\hline 4. & 7.31 & .627 & 1.70 & 4.59 & 6.73 & 1.10 & 2.84 & 7.38 & .921 & 2.81 \\
\hline 4.5 & 8.23 & .570 & 1.64 & 4.69 & 7.62 & .964 & 2.66 & 7.34 & .925 & 2.64 \\
\hline 5. & 9.15 & .523 & 1.58 & 4.79 & 8.51 & .858 & 2.50 & 7.30 & .929 & 2.50 \\
\hline \multicolumn{11}{|c|}{$\left\langle\frac{5}{3}, .6\right\rangle$} \\
\hline 0 & 1 & 0 & 0 & 0 & 0 & $\infty$ & $\infty$ & 6.28 & 0 & $\infty$ \\
\hline .1 & 1.02 & .430 & .433 & .437 & .124 & 52.2 & 18.4 & 6.45 & .122 & 1790 . \\
\hline .3 & 1.14 & 1.09 & 1.16 & 1.24 & .396 & 17.1 & 10.7 & 6.76 & .348 & 85.3 \\
\hline .5 & 1.36 & 1.40 & 1.63 & 1.90 & .705 & 9.93 & 8.34 & 7.00 & .520 & 26.1 \\
\hline .7 & 1.63 & 1.47 & 1.88 & 2.41 & 1.04 & 6.87 & 7.02 & 7.16 & .639 & 13.9 \\
\hline 1. & 2.11 & 1.40 & 2.04 & 2.96 & 1.59 & 4.61 & 5.80 & 7.30 & .751 & 8.11 \\
\hline 1.5 & 3.00 & 1.18 & 2.05 & 3.55 & 2.54 & 2.91 & 4.63 & 7.38 & .847 & 5.10 \\
\hline 2. & 3.93 & .999 & 1.98 & 3.93 & 3.52 & 2.10 & 3.94 & 7.39 & .895 & 3.96 \\
\hline 2.5 & 4.89 & .857 & 1.89 & 4.19 & 4.51 & 1.63 & 3.47 & 7.37 & .922 & 3.36 \\
\hline 3. & 5.86 & .749 & 1.81 & 4.38 & 5.51 & 1.33 & 3.13 & 7.34 & .940 & 2.98 \\
\hline 3.5 & 6.84 & .664 & 1.74 & 4.54 & 6.50 & 1.12 & 2.86 & 7.30 & .951 & 2.73 \\
\hline 4. & 7.82 & .596 & 1.67 & 4.66 & 7.51 & .970 & 2.66 & 7.28 & .960 & 2.54 \\
\hline 4.5 & 8.80 & .540 & 1.60 & 4.76 & 8.51 & .852 & 2.48 & 7.25 & .967 & 2.40 \\
\hline 5. & 9.79 & .494 & 1.55 & 4.84 & 9.50 & .759 & 2.34 & 7.22 & .971 & 2.29 \\
\hline
\end{tabular}


$\left\langle\frac{5}{3}, .8\right\rangle$

\begin{tabular}{|c|c|c|c|c|c|c|c|c|c|c|}
\hline$x$ & $r$ & a & $A_{0}$ & $A$ & $r^{\prime}$ & $Q^{\prime}$ & $A_{0}^{\prime}$ & $A^{\prime}$ & $\mathbf{\Omega}$ & $Q$ \\
\hline 0 & 1 & 0 & 0 & 0 & 0 & $\infty$ & $\infty$ & 6.28 & 0 & $\infty$ \\
\hline .1 & 1.02 & .470 & .474 & .478 & .162 & 39.2 & 15.8 & 6.37 & .159 & 1110. \\
\hline .3 & 1.16 & 1.17 & 1.26 & 1.36 & .503 & 13.0 & 9.20 & 6.52 & .434 & 53.0 \\
\hline .5 & 1.41 & 1.47 & 1.74 & 2.07 & .867 & 7.67 & 7.15 & 6.65 & .614 & 16.8 \\
\hline .7 & 1.74 & 1.51 & 1.99 & 2.62 & 1.25 & 5.39 & 6.03 & 6.74 & .720 & 9.18 \\
\hline 1. & 2.32 & 1.39 & 2.12 & 3.22 & 1.85 & 3.69 & 5.02 & 6.83 & .800 & 5.62 \\
\hline 1.5 & 3.39 & 1.14 & 2.09 & 3.85 & 2.89 & 2.38 & 4.05 & 6.89 & .853 & 3.76 \\
\hline 2. & 4.53 & .936 & 1.99 & 4.24 & 3.94 & 1.75 & 3.47 & 6.89 & .871 & 3.03 \\
\hline 2.5 & 5.70 & .790 & 1.89 & 4.51 & 5.00 & 1.38 & 3.08 & 6.89 & .877 & 2.67 \\
\hline 3. & 6.90 & .682 & 1.79 & 4.71 & 6.07 & 1.13 & 2.78 & 6.86 & .879 & 2.41 \\
\hline 3.5 & 8.12 & .599 & 1.71 & 4.86 & 7.13 & .959 & 2.56 & 6.83 & .878 & 2.25 \\
\hline 4. & 9.34 & .534 & 1.63 & 4.99 & 8.19 & .832 & 2.38 & 6.81 & .876 & 2.13 \\
\hline 4.5 & 10.6 & .481 & 1.56 & 5.09 & 9.24 & .734 & 2.23 & 6.78 & .873 & 2.03 \\
\hline 5. & 11.8 & .438 & 1.50 & 5.18 & 10.3 & .658 & 2.11 & 6.76 & .870 & 1.97 \\
\hline
\end{tabular}

$\left\langle\frac{5}{3}, 1.05\right\rangle$

\begin{tabular}{|c|c|c|c|c|c|c|c|c|c|c|}
\hline 0 & 1 & 0 & 0 & 0 & 0 & $\infty$ & $\infty$ & 6.28 & 0 & $\infty$ \\
\hline .1 & 1.02 & .519 & .525 & .530 & .209 & 29.9 & 13.7 & 6.26 & .205 & 680. \\
\hline .3 & 1.19 & 1.28 & 1.39 & 1.52 & .623 & 9.99 & 7.89 & 6.22 & .525 & 32.1 \\
\hline .5 & 1.49 & 1.57 & 1.91 & 2.33 & 1.03 & 6.01 & 6.10 & 6.19 & .692 & 10.2 \\
\hline .7 & 1.90 & 1.56 & 2.15 & 2.96 & 1.43 & 4.31 & 5.15 & 6.16 & .754 & 5.73 \\
\hline 1. & 2.64 & 1.38 & 2.25 & 3.66 & 2.02 & 3.04 & 4.32 & 6.14 & .764 & 3.69 \\
\hline 1.5 & 4.10 & 1.07 & 2.16 & 4.37 & 2.99 & 2.05 & 3.54 & 6.13 & .730 & 2.69 \\
\hline 2. & 5.69 & .842 & 2.01 & 4.79 & 3.97 & 1.55 & 3.08 & 6.14 & .698 & 2.35 \\
\hline 2.5 & 7.34 & .690 & 1.87 & 5.06 & 4.95 & 1.24 & 2.76 & 6.15 & .675 & 2.19 \\
\hline 3. & 9.02 & .582 & 1.75 & 5.25 & 5.94 & 1.04 & 2.53 & 6.16 & .658 & 2.10 \\
\hline 3.5 & 10.7 & .503 & 1.65 & 5.39 & 6.93 & .892 & 2.35 & 6.18 & .646 & 2.03 \\
\hline 4. & 12.4 & .442 & 1.56 & 5.49 & 7.92 & .782 & 2.20 & 6.19 & .637 & 1.99 \\
\hline 4.5 & 14.2 & .394 & 1.48 & 5.58 & 8.91 & .695 & 2.08 & 6.20 & .630 & 1.96 \\
\hline 5. & 15.9 & .356 & 1.42 & 5.64 & 9.91 & .626 & 1.97 & 6.20 & .624 & 1.93 \\
\hline \multicolumn{11}{|c|}{$\left\langle\frac{5}{3}, 1.25\right\rangle$} \\
\hline 0 & 1 & 0 & 0 & 0 & 0 & $\infty$ & $\infty$ & 6.28 & 0 & $\infty$ \\
\hline .1 & 1.02 & .560 & .566 & .573 & .246 & 25.2 & 12.5 & 6.18 & .240 & 484. \\
\hline .3 & 1.20 & 1.37 & 1.50 & 1.65 & .709 & 8.44 & 7.10 & 5.98 & .588 & 22.3 \\
\hline .5 & 1.55 & 1.65 & 2.06 & 2.57 & 1.13 & 5.13 & 5.45 & 5.79 & .727 & 6.99 \\
\hline .7 & 2.06 & 1.61 & 2.31 & 3.31 & 1.51 & 3.74 & 4.59 & 5.64 & .733 & 3.95 \\
\hline 1. & 3.04 & 1.35 & 2.36 & 4.11 & 2.02 & 2.73 & 3.89 & 5.53 & .664 & 2.72 \\
\hline 1.5 & 4.97 & .970 & 2.16 & 4.82 & 2.88 & 1.93 & 3.28 & 5.58 & .580 & 2.30 \\
\hline 2. & 7.00 & .741 & 1.96 & 5.18 & 3.80 & 1.50 & 2.91 & 5.68 & .543 & 2.21 \\
\hline 2.5 & 9.04 & .597 & 1.80 & 5.40 & 4.75 & 1.22 & 2.65 & 5.78 & .525 & 2.18 \\
\hline 3. & 11.1 & .499 & 1.66 & 5.54 & 5.71 & 1.02 & 2.44 & 5.84 & .514 & 2.16 \\
\hline 3.5 & 13.2 & .429 & 1.56 & 5.65 & 6.68 & .882 & 2.28 & 5.90 & .508 & 2.15 \\
\hline 4. & 15.2 & .375 & 1.47 & 5.72 & 7.67 & .775 & 2.15 & 5.94 & .503 & 2.14 \\
\hline 4.5 & 17.3 & .334 & 1.39 & 5.79 & 8.65 & .691 & 2.03 & 5.98 & .500 & 2.13 \\
\hline 5. & 19.4 & .301 & 1.32 & 5.83 & 9.64 & .623 & 1.93 & 6.01 & .497 & 2.13 \\
\hline
\end{tabular}


$\langle 1.8, .3\rangle$

\begin{tabular}{|c|c|c|c|c|c|c|c|c|c|c|}
\hline$X$ & $r$ & a & $A_{0}$ & $A$ & $r^{\prime}$ & $Q^{\prime}$ & $A_{0}^{\prime}$ & $A^{\prime}$ & $\Omega$ & $Q$ \\
\hline 0 & 1 & 0 & 0 & 0 & 0 & $\infty$ & $\infty$ & 6.28 & 0 & $\infty$ \\
\hline .1 & 1.01 & .382 & .385 & .387 & .0639 & 104. & 26.3 & 6.64 & .0631 & 4660 . \\
\hline .3 & 1.11 & .994 & 1.05 & 1.11 & .222 & 32.8 & 15.4 & 7.28 & .200 & 217. \\
\hline .5 & 1.29 & 1.32 & 1.50 & 1.70 & .422 & 18.3 & 11.9 & 7.71 & .328 & 62.5 \\
\hline .7 & 1.51 & 1.44 & 1.77 & 2.18 & .652 & 12.2 & 9.84 & 7.94 & .432 & 30.8 \\
\hline 1. & 1.90 & 1.43 & 1.97 & 2.72 & 1.02 & 7.88 & 7.97 & 8.06 & .539 & 16.3 \\
\hline 1.5 & 2.63 & 1.27 & 2.05 & 3.33 & 1.66 & 4.82 & 6.22 & 8.02 & .633 & 9.17 \\
\hline 2. & 3.41 & 1.10 & 2.03 & 3.74 & 2.30 & 3.44 & 5.21 & 7.89 & .674 & 6.60 \\
\hline 2.5 & 4.22 & .956 & 1.96 & 4.04 & 2.92 & 2.66 & 4.54 & 7.76 & .692 & 5.34 \\
\hline 3. & 5.06 & .844 & 1.90 & 4.27 & 3.54 & 2.16 & 4.06 & 7.64 & .699 & 4.58 \\
\hline 3.5 & 5.92 & .752 & 1.83 & 4.45 & 4.14 & 1.82 & 3.70 & 7.53 & .700 & 4.08 \\
\hline 4. & 6.79 & .678 & 1.77 & 4.61 & 4.74 & 1.57 & 3.42 & 7.44 & .698 & 3.74 \\
\hline 4.5 & 7.68 & .616 & 1.71 & 4.73 & 5.33 & 1.38 & 3.18 & 7.35 & .694 & 3.47 \\
\hline 5. & 8.58 & .565 & 1.65 & 4.85 & 5.91 & 1.23 & 2.99 & 7.28 & .689 & 3.27 \\
\hline
\end{tabular}

$\langle 1.8, .5\rangle$

\begin{tabular}{l|l|l|l|l|l|r|r|r|r|r}
\hline 0 & 1 & 0 & 0 & 0 & 0 & $\infty$ & $\infty$ & 6.28 & 0 & $\infty$ \\
.1 & 1.02 & .429 & .432 & .436 & .105 & 62.5 & 20.2 & 6.54 & .103 & 2190. \\
.3 & 1.14 & 1.08 & 1.16 & 1.24 & .347 & 20.1 & 11.9 & 6.99 & .305 & 105. \\
.5 & 1.36 & 1.38 & 1.61 & 1.88 & .637 & 11.5 & 9.17 & 7.32 & .470 & 32.4 \\
.7 & 1.63 & 1.46 & 1.86 & 2.37 & .961 & 7.83 & 7.67 & 7.52 & .590 & 17.0 \\
1. & 2.09 & 1.39 & 2.01 & 2.91 & 1.48 & 5.16 & 6.29 & 7.66 & .709 & 9.80 \\
1.5 & 2.95 & 1.18 & 2.03 & 3.48 & 2.40 & 3.21 & 4.98 & 7.71 & .815 & 6.02 \\
2. & 3.84 & 1.00 & 1.97 & 3.85 & 3.34 & 2.30 & 4.20 & 7.68 & .869 & 4.57 \\
2.5 & 4.76 & .865 & 1.89 & 4.11 & 4.28 & 1.78 & 3.68 & 7.63 & .900 & 3.82 \\
3. & 5.68 & .758 & 1.81 & 4.31 & 5.23 & 1.45 & 3.31 & 7.57 & .920 & 3.36 \\
3.5 & 6.62 & .674 & 1.73 & 4.46 & 6.17 & 1.22 & 3.03 & 7.52 & .933 & 3.05 \\
4. & 7.55 & .607 & 1.67 & 4.58 & 7.12 & 1.05 & 2.80 & 7.47 & .942 & 2.82 \\
4.5 & 8.50 & .551 & 1.61 & 4.68 & 8.06 & .921 & 2.62 & 7.43 & .949 & 2.65 \\
5. & 9.44 & .506 & 1.55 & 4.77 & 9.01 & .820 & 2.46 & 7.38 & .954 & 2.51 \\
\hline & & & & & $<2, .3>$ & & & & \\
& & & & & & & & & \\
\hline 0 & 1 & 0 & 0 & 0 & 0 & $\infty$ & $\infty$ & 6.28 & 0 & $\infty$ \\
.1 & 1.02 & .399 & .402 & .405 & .0650 & 104. & 26.4 & 6.73 & .0641 & 4310. \\
.3 & 1.12 & 1.02 & 1.08 & 1.15 & .235 & 32.0 & 15.5 & 7.52 & .209 & 205. \\
.5 & 1.31 & 1.34 & 1.53 & 1.75 & .458 & 17.5 & 11.8 & 8.02 & .349 & 59.8 \\
.7 & 1.55 & 1.44 & 1.79 & 2.23 & .715 & 11.5 & 9.76 & 8.25 & .461 & 29.8 \\
1. & 1.96 & 1.41 & 1.97 & 2.75 & 1.13 & 7.39 & 7.86 & 8.36 & .577 & 15.9 \\
1.5 & 2.71 & 1.23 & 2.03 & 3.34 & 1.84 & 4.49 & 6.10 & 8.27 & .680 & 9.02 \\
2. & 3.51 & 1.06 & 1.99 & 3.73 & 2.55 & 3.19 & 5.08 & 8.12 & .727 & 6.51 \\
2.5 & 4.33 & .927 & 1.93 & 4.02 & 3.25 & 2.46 & 4.43 & 7.98 & .749 & 5.26 \\
3. & 5.17 & .818 & 1.86 & 4.23 & 3.93 & 1.99 & 3.95 & 7.84 & .760 & 4.50 \\
3.5 & 6.03 & .731 & 1.80 & 4.41 & 4.61 & 1.68 & 3.60 & 7.72 & .764 & 4.02 \\
4. & 6.91 & .660 & 1.73 & 4.56 & 5.28 & 1.44 & 3.32 & 7.62 & .765 & 3.66 \\
4.5 & 7.79 & .601 & 1.68 & 4.68 & 5.95 & 1.27 & 3.09 & 7.53 & .763 & 3.39 \\
5. & 8.68 & .551 & 1.62 & 4.78 & 6.60 & 1.13 & 2.90 & 7.45 & .761 & 3.19 \\
\hline & & & & & & & & & & \\
& & & & & & & & & \\
\end{tabular}


$\langle 2, .5\rangle$

\begin{tabular}{|c|c|c|c|c|c|c|c|c|c|c|}
\hline$x$ & $r$ & a & $A_{0}$ & $A$ & $r^{\prime}$ & $a^{\prime}$ & $A_{0}^{\prime}$ & $A^{\prime}$ & $\Omega$ & $Q$ \\
\hline 0 & 1 & 0 & 0 & 0 & 0 & $\infty$ & $\infty$ & 6.28 & 0 & $\infty$ \\
\hline .1 & 1.02 & .458 & .462 & .467 & .106 & 62.4 & 20.3 & 6.60 & .104 & 1930. \\
\hline .3 & 1.16 & 1.13 & 1.21 & 1.31 & .361 & 19.8 & 11.9 & 7.16 & .311 & 96.2 \\
\hline .5 & 1.40 & 1.40 & 1.66 & 1.97 & .674 & 11.2 & 9.16 & 7.52 & .481 & 30.5 \\
\hline .7 & 1.70 & 1.45 & 1.89 & 2.46 & 1.03 & 7.53 & 7.63 & 7.72 & .603 & 16.4 \\
\hline 1. & 2.20 & 1.35 & 2.01 & 2.98 & 1.59 & 4.93 & 6.22 & 7.85 & .723 & 9.58 \\
\hline 1.5 & 3.11 & 1.14 & 2.01 & 3.54 & 2.58 & 3.05 & 4.90 & 7.86 & .828 & 5.94 \\
\hline 2. & 4.06 & .961 & 1.94 & 3.90 & 3.58 & 2.18 & 4.12 & 7.80 & .881 & 4.54 \\
\hline 2.5 & 5.02 & .826 & 1.85 & 4.15 & 4.58 & 1.69 & 3.61 & 7.73 & .912 & 3.81 \\
\hline 3. & 6.00 & .723 & 1.77 & 4.34 & 5.59 & 1.37 & 3.24 & 7.66 & .932 & 3.35 \\
\hline 3.5 & 6.98 & .642 & 1.70 & 4.48 & 6.60 & 1.15 & 2.96 & 7.60 & .946 & 3.04 \\
\hline 4. & 7.96 & .578 & 1.63 & 4.60 & 7.60 & .993 & 2.74 & 7.55 & .955 & 2.82 \\
\hline 4.5 & 8.95 & .525 & 1.57 & 4.70 & 8.61 & .871 & 2.56 & 7.50 & .962 & 2.65 \\
\hline 5. & 9.94 & .481 & 1.52 & 4.78 & 9.61 & .775 & 2.40 & 7.45 & .967 & 2.51 \\
\hline
\end{tabular}

$\langle 2.2, .3\rangle$

\begin{tabular}{|c|c|c|c|c|c|c|c|c|c|c|}
\hline 0 & 1 & 0 & 0 & 0 & 0 & $\infty$ & $\infty$ & 6.28 & 0 & $\infty$ \\
\hline .1 & 1.02 & .417 & .420 & .423 & .0661 & 103. & 26.5 & 6.82 & .0651 & 3990 . \\
\hline .3 & 1.14 & 1.05 & 1.12 & 1.19 & .248 & 31.3 & 15.6 & 7.75 & .218 & 194 \\
\hline .5 & 1.34 & 1.35 & 1.56 & 1.80 & .494 & 16.8 & 11.8 & 8.29 & .369 & 57.3 \\
\hline .7 & 1.59 & 1.43 & 1.80 & 2.27 & .778 & 10.9 & 9.66 & 8.52 & .489 & 28.7 \\
\hline 1. & 2.02 & 1.38 & 1.96 & 2.79 & 1.24 & 6.96 & 7.73 & 8.60 & .612 & 15.5 \\
\hline 1.5 & 2.79 & 1.20 & 2.01 & 3.36 & 2.01 & 4.21 & 5.97 & 8.48 & .721 & 8.83 \\
\hline 2. & 3.61 & 1.03 & 1.96 & 3.73 & 2.78 & 2.98 & 4.97 & 8.30 & .771 & 6.41 \\
\hline 2.5 & 4.45 & .900 & 1.90 & 4.00 & 3.55 & 2.29 & 4.32 & 8.14 & .798 & 5.18 \\
\hline 3. & 5.30 & .794 & 1.83 & 4.21 & 4.30 & 1.86 & 3.86 & 8.00 & .812 & 4.44 \\
\hline 3.5 & 6.17 & .710 & 1.76 & 4.38 & 5.05 & 1.56 & 3.51 & 7.88 & .818 & 3.95 \\
\hline 4. & 7.05 & .641 & 1.70 & 4.52 & 5.79 & 1.34 & 3.23 & 7.77 & .821 & 3.60 \\
\hline 4.5 & 7.93 & .584 & 1.64 & 4.63 & 6.52 & 1.18 & 3.01 & 7.68 & .823 & 3.34 \\
\hline 5. & 8.82 & .537 & 1.59 & 4.73 & 7.25 & 1.05 & 2.82 & 7.60 & .822 & 3.13 \\
\hline \multicolumn{11}{|c|}{$\langle 2.2, .5\rangle$} \\
\hline 0 & 1 & 0 & 0 & 0 & 0 & $\infty$ & $\infty$ & 6.28 & 0 & $\infty$ \\
\hline .1 & 1.02 & .486 & .492 & .497 & .107 & 62.2 & 20.4 & 6.66 & .105 & 1720. \\
\hline .3 & 1.18 & 1.17 & 1.27 & 1.38 & .375 & 19.5 & 11.9 & 7.31 & .318 & 88.6 \\
\hline .5 & 1.45 & 1.42 & 1.70 & 2.05 & .711 & 10.8 & 9.13 & 7.70 & .491 & 28.8 \\
\hline .7 & 1.77 & 1.43 & 1.91 & 2.54 & 1.09 & 7.25 & 7.57 & 7.89 & .614 & 15.7 \\
\hline 1. & 2.31 & 1.32 & 2.01 & 3.06 & 1.69 & 4.72 & 6.14 & 7.98 & .731 & 9.30 \\
\hline 1.5 & 3.28 & 1.10 & 1.99 & 3.61 & 2.73 & 2.91 & 4.81 & 7.95 & .832 & 5.84 \\
\hline 2. & 4.28 & .923 & 1.91 & 3.96 & 3.78 & 2.08 & 4.05 & 7.87 & .882 & 4.49 \\
\hline 2.5 & 5.30 & .792 & 1.82 & 4.20 & 4.84 & 1.61 & 3.54 & 7.79 & .912 & 3.78 \\
\hline 3. & 6.33 & .692 & 1.74 & 4.38 & 5.88 & 1.31 & 3.18 & 7.70 & .930 & 3.33 \\
\hline 3.5 & 7.37 & .614 & 1.67 & 4.52 & 6.93 & 1.10 & 2.90 & 7.63 & .941 & 3.02 \\
\hline 4. & 8.41 & .552 & 1.60 & 4.64 & 7.98 & .948 & 2.68 & 7.57 & .949 & 2.80 \\
\hline 4.5 & 9.45 & .501 & 1.54 & 4.74 & 9.03 & .832 & 2.50 & 7.51 & .955 & 2.64 \\
\hline 5. & 10.5 & .459 & 1.49 & 4.82 & 10.1 & .741 & 2.35 & 7.46 & .960 & 2.50 \\
\hline
\end{tabular}


Group II. Strong conductors $\langle 1.10,20\rangle$

\begin{tabular}{l|c|c|c|c|c|c|c|c|c|c}
\hline$X$ & \multicolumn{1}{|c|}{$\alpha$} & \multicolumn{1}{|c|}{$Q$} & \multicolumn{1}{|c|}{$A_{0}$} & \multicolumn{1}{|c|}{$A$} & $r^{\prime}$ & $Q^{\prime}$ & $A_{0}^{\prime}$ & $A^{\prime}$ & $\Omega$ & $Q$ \\
\hline 0 & 1 & 0 & 0 & 0 & 0 & $\infty$ & $\infty$ & 6.28 & 0 & $\infty$ \\
.1 & .934 & .495 & .478 & .462 & 4.18 & 1.45 & 2.96 & 6.06 & 4.47 & 38.4 \\
.3 & .986 & 1.07 & 1.06 & 1.06 & 13.0 & .477 & 1.72 & 6.20 & 13.2 & 2.61 \\
.5 & 1.11 & 1.48 & 1.56 & 1.64 & 21.8 & .286 & 1.33 & 6.23 & 19.7 & .731 \\
.7 & 1.28 & 1.68 & 1.91 & 2.16 & 30.6 & .204 & 1.13 & 6.24 & 23.8 & .350 \\
1. & 1.62 & 1.73 & 2.20 & 2.79 & 43.8 & .143 & .945 & 6.26 & 27.0 & .185 \\
1.5 & 2.32 & 1.53 & 2.34 & 3.55 & 65.8 & .0952 & .772 & 6.26 & 28.4 & .109 \\
2. & 3.11 & 1.30 & 2.30 & 4.06 & 87.8 & .0714 & .669 & 6.27 & 28.2 & .0848 \\
2.5 & 3.97 & 1.11 & 2.21 & 4.41 & 110. & .0571 & .598 & 6.27 & 27.7 & .0731 \\
3. & 4.87 & .959 & 2.12 & 4.67 & 132. & .0476 & .546 & 6.27 & 27.1 & .0667 \\
3.5 & 5.79 & .841 & 2.02 & 4.87 & 154. & .0408 & .506 & 6.28 & 26.6 & .0626 \\
4. & 6.72 & .747 & 1.94 & 5.02 & 176. & .0357 & .473 & 6.28 & 26.2 & .0597 \\
4.5 & 7.67 & .671 & 1.86 & 5.15 & 198. & .0317 & .446 & 6.28 & 25.8 & .0576 \\
5. & 8.63 & .608 & 1.79 & 5.25 & 220. & .0286 & .424 & 6.28 & 25.5 & .0562 \\
\hline
\end{tabular}

\begin{tabular}{|c|c|c|c|c|c|c|c|c|c|c|}
\hline \multicolumn{11}{|c|}{$\langle 1.10,30\rangle$} \\
\hline 0 & 1 & 0 & 0 & 0 & 0 & $\infty$ & $\infty$ & 6.28 & 0 & $\infty$ \\
\hline .1 & .926 & .463 & .446 & .429 & 6.38 & .959 & 2.42 & 6.12 & 6.90 & 29.5 \\
\hline .3 & .984 & 1.06 & 1.05 & 1.04 & 19.6 & .318 & 1.41 & 6.22 & 19.9 & 1.80 \\
\hline .5 & 1.11 & 1.47 & 1.55 & 1.63 & 32.8 & .190 & 1.09 & 6.25 & 29.6 & .494 \\
\hline .7 & 1.28 & 1.68 & 1.90 & 2.15 & 46.0 & .136 & .923 & 6.26 & 35.9 & .236 \\
\hline 1. & 1.62 & 1.72 & 2.19 & 2.79 & 65.8 & .0952 & .772 & 6.26 & 40.7 & .124 \\
\hline 1.5 & 2.31 & 1.53 & 2.33 & 3.55 & 98.8 & .0635 & .631 & 6.27 & 42.7 & .0732 \\
\hline 2. & 3.11 & 1.30 & 2.30 & 4.05 & 132. & .0476 & .546 & 6.27 & 42.4 & .0566 \\
\hline 2.5 & 3.97 & 1.11 & 2.21 & 4.40 & 165. & .0381 & .489 & 6.28 & 41.5 & .0489 \\
\hline 3. & 4.86 & .959 & 2.12 & 4.67 & 198. & .0317 & .446 & 6.28 & 40.7 & .0445 \\
\hline 3.5 & 5.78 & .841 & 2.02 & 4.86 & 231. & .0272 & .413 & 6.28 & 39.9 & .0417 \\
\hline 4. & 6.72 & .747 & 1.94 & 5.02 & 264 & .0238 & .386 & 6.28 & 39.2 & .0398 \\
\hline 4.5 & 7.67 & .671 & 1.86 & 5.14 & 297. & .0212 & .365 & 6.28 & 38.7 & .0383 \\
\hline 5. & 8.63 & .608 & 1.79 & 5.25 & 330. & .0190 & .346 & 6.28 & 38.2 & .0372 \\
\hline \multicolumn{11}{|c|}{$\langle 1.10,40\rangle$} \\
\hline 0 & 1 & 0 & 0 & 0 & 0 & $\infty$ & $\infty$ & 6.28 & 0 & $\infty$ \\
\hline .1 & .922 & .443 & .426 & .409 & 8.59 & .717 & 2.10 & 6.16 & 9.31 & 24.4 \\
\hline .3 & .984 & 1.05 & 1.04 & 1.03 & 26.2 & .238 & 1.22 & 6.24 & 26.6 & 1.37 \\
\hline .5 & 1.11 & 1.47 & 1.55 & 1.63 & 43.8 & .143 & .945 & 6.26 & 39.6 & .373 \\
\hline .7 & 1.28 & 1.68 & 1.90 & 2.15 & 61.4 & .102 & .799 & 6.26 & 47.9 & .177 \\
\hline 1. & 1.62 & 1.72 & 2.19 & 2.79 & 87.8 & .0714 & .669 & 6.27 & 54.3 & .0932 \\
\hline 1.5 & 2.31 & 1.53 & 2.33 & 3.54 & 132. & .0476 & .546 & 6.27 & 57.0 & .0549 \\
\hline 2. & 3.11 & 1.30 & 2.30 & 4.05 & 176. & .0357 & .473 & 6.27 & 56.5 & .0424 \\
\hline 2.5 & 3.97 & 1.11 & 2.21 & 4.40 & 220 . & .0286 & .424 & 6.28 & 55.4 & .0367 \\
\hline 3. & 4.86 & .959 & 2.12 & 4.66 & 264. & .0238 & .386 & 6.28 & 54.2 & .0334 \\
\hline 3.5 & 5.78 & .841 & 2.02 & 4.86 & 308. & .0204 & .358 & 6.28 & 53.2 & .0313 \\
\hline 4. & 6.72 & .747 & 1.94 & 5.02 & 352. & .0178 & .335 & 6.28 & 52.4 & .0299 \\
\hline 4.5 & 7.67 & .671 & 1.86 & 5.14 & 396. & .0159 & .316 & 6.28 & 51.6 & .0289 \\
\hline 5. & 8.62 & .608 & 1.79 & 5.24 & 440. & .0143 & .300 & 6.28 & 51.0 & .0279 \\
\hline
\end{tabular}




\begin{tabular}{|c|c|c|c|c|c|c|c|c|c|c|}
\hline \multicolumn{11}{|c|}{$\langle 1.15,10\rangle$} \\
\hline$x$ & $r$ & $a$ & $A_{0}$ & $A$ & $r^{\prime}$ & $a^{\prime}$ & $A_{0}^{\prime}$ & $A^{\prime}$ & $\mathbf{\Omega}$ & $Q$ \\
\hline 0 & 1 & 0 & 0 & 0 & 0 & $\infty$ & $\infty$ & 6.28 & 0 & $\infty$ \\
\hline .1 & .944 & .635 & .617 & .600 & 2.00 & 2.91 & 4.12 & 5.82 & 2.12 & 44.5 \\
\hline .3 & .962 & 1.23 & 1.20 & 1.18 & 6.57 & .919 & 2.36 & 6.04 & 6.83 & 3.83 \\
\hline .5 & 1.09 & 1.62 & 1.69 & 1.76 & 11.2 & .548 & 1.83 & 6.13 & 10.3 & 1.18 \\
\hline .7 & 1.26 & 1.80 & 2.02 & 2.28 & 15.8 & .391 & 1.55 & 6.17 & 12.5 & .588 \\
\hline 1. & 1.61 & 1.81 & 2.29 & 2.91 & 22.7 & .273 & 1.30 & 6.21 & 14.1 & .323 \\
\hline 1.5 & 2.32 & 1.58 & 2.40 & 3.66 & 34.2 & .182 & 1.07 & 6.23 & 14.7 & .197 \\
\hline 2. & 3.13 & 1.32 & 2.34 & 4.15 & 45.7 & .137 & .924 & 6.25 & 14.6 & .156 \\
\hline 2.5 & 4.00 & 1.12 & 2.24 & 4.49 & 57.2 & .109 & .827 & 6.25 & 14.3 & .136 \\
\hline 3. & 4.90 & .967 & 2.14 & 4.74 & 68.7 & .0910 & .755 & 6.26 & 14.0 & .124 \\
\hline 3.5 & 5.83 & .846 & 2.04 & 4.93 & 80.3 & .0781 & .699 & 6.26 & 13.8 & .117 \\
\hline 4. & 6.77 & .750 & 1.95 & 5.08 & 91.7 & .0683 & .654 & 6.26 & 13.6 & .112 \\
\hline 4.5 & 7.72 & .673 & 1.87 & 5.20 & 103. & .0607 & .617 & 6.26 & 13.4 & .109 \\
\hline 5. & 8.68 & .610 & 1.80 & 5.30 & 115. & .0546 & .585 & 6.27 & 13.2 & .106 \\
\hline \multicolumn{11}{|c|}{$\langle 1.15,20\rangle$} \\
\hline 0 & 1 & 0 & 0 & 0 & 0 & $\infty$ & $\infty$ & 6.28 & 0 & $\infty$ \\
\hline .1 & .899 & .586 & .556 & $.5 \dot{2} 7$ & 4.28 & 1.39 & 2.88 & 5.96 & 4.76 & 26.8 \\
\hline .3 & .951 & 1.17 & 1.14 & 1.12 & 13.5 & .456 & 1.68 & 6.16 & 14.2 & 2.15 \\
\hline .5 & 1.08 & 1.59 & 1.65 & 1.72 & 22.7 & .274 & 1.30 & 6.21 & 21.0 & .621 \\
\hline .7 & 1.26 & 1.78 & 2.00 & 2.24 & 31.9 & .195 & 1.10 & 6.23 & 25.3 & .304 \\
\hline 1. & 1.60 & 1.80 & 2.28 & 2.89 & 45.7 & .137 & .924 & 6.24 & 28.5 & .164 \\
\hline 1.5 & 2.32 & 1.57 & 2.39 & 3.64 & 68.7 & .0911 & .755 & 6.26 & 29.7 & .0997 \\
\hline 2. & 3.13 & 1.32 & 2.34 & 4.14 & 91.7 & .0683 & .654 & 6.26 & 29.3 & .0782 \\
\hline 2.5 & 3.99 & 1.12 & 2.24 & 4.48 & 115. & .0546 & .585 & 6.27 & 28.8 & .0682 \\
\hline 3. & 4.89 & .966 & 2.14 & 4.73 & 138. & .0455 & .534 & 6.27 & 28.1 & .0624 \\
\hline 3.5 & 5.82 & .846 & 2.04 & 4.92 & 161. & .0390 & .495 & 6.27 & 27.6 & .0588 \\
\hline 4. & 6.76 & .750 & 1.95 & 5.07 & 184. & .0342 & .463 & 6.27 & 27.2 & .0563 \\
\hline 4.5 & 7.72 & .673 & 1.87 & 5.19 & 207. & .0304 & .437 & 6.28 & 26.8 & .0546 \\
\hline 5. & 8.67 & .610 & 1.80 & 5.29 & 230 . & .0273 & .414 & 6.28 & 26.5 & .0532 \\
\hline \multicolumn{11}{|c|}{$\langle 1.15,25\rangle$} \\
\hline 0 & 1 & 0 & 0 & 0 & 0 & $\infty$ & $\infty$ & 6.28 & 0 & $\infty$ \\
\hline .1 & .892 & .559 & .528 & .498 & 5.44 & 1.11 & 2.58 & 6.01 & 6.09 & 23.9 \\
\hline .3 & .950 & 1.16 & 1.13 & 1.10 & 17.0 & .365 & 1.50 & 6.18 & 17.9 & 1.76 \\
\hline .5 & 1.08 & 1.59 & 1.65 & 1.71 & 28.5 & .219 & 1.17 & 6.23 & 26.4 & .503 \\
\hline .7 & 1.26 & 1.78 & 2.00 & 2.24 & 40.0 & .156 & .987 & 6.24 & 31.8 & .244 \\
\hline 1. & 1.60 & 1.80 & 2.27 & 2.88 & 57.2 & .109 & .827 & 6.26 & 35.7 & .132 \\
\hline 1.5 & 2.31 & 1.57 & 2.39 & 3.63 & 86.0 & .0729 & .676 & 6.27 & 37.2 & .0801 \\
\hline 2. & 3.12 & 1.32 & 2.34 & 4.13 & 115. & .0546 & .585 & 6.27 & 36.7 & .0628 \\
\hline 2.5 & 3.99 & 1.12 & 2.24 & 4.48 & 144. & .0437 & .524 & 6.27 & 36.0 & .0546 \\
\hline 3. & 4.89 & .966 & 2.14 & 4.73 & 172. & .0364 & .478 & 6.27 & 35.2 & .0499 \\
\hline 3.5 & 5.82 & .846 & 2.04 & 4.92 & 201 & .0312 & .443 & 6.28 & 34.6 & .0472 \\
\hline 4. & 6.76 & .750 & 1.95 & 5.07 & 230. & .0273 & .414 & 6.28 & 34.0 & .0452 \\
\hline 4.5 & 7.71 & .673 & 1.87 & 5.19 & 259 . & .0243 & .390 & 6.28 & 33.5 & .0436 \\
\hline 5. & 8.67 & .610 & 1.80 & 5.29 & 287. & .0219 & .370 & 6.28 & 33.1 & .0426 \\
\hline
\end{tabular}


$\langle 1.15,30\rangle$

\begin{tabular}{|c|c|c|c|c|c|c|c|c|c|c|}
\hline $\boldsymbol{X}$ & $r$ & $a$ & $A_{0}$ & $A$ & $r^{\prime}$ & $Q^{\prime}$ & $A_{0}^{\prime}$ & $A^{\prime}$ & $\mathbf{\Omega}$ & $Q$ \\
\hline 0 & 1 & 0 & 0 & 0 & 0 & $\infty$ & $\infty$ & 6.28 & 0 & $\infty$ \\
\hline .1 & .888 & .537 & .506 & .477 & 6.59 & .918 & 2.36 & 6.06 & 7.42 & 21.7 \\
\hline .3 & .949 & 1.15 & 1.12 & 1.10 & 20.4 & .304 & 1.37 & 6.20 & 21.5 & 1.49 \\
\hline .5 & 1.08 & 1.58 & 1.64 & 1.70 & 34.2 & 182 & 1.07 & 6.24 & 31.8 & .422 \\
\hline .7 & 1.26 & 1.78 & 1.99 & 2.24 & 48.0 & .130 & .902 & 6.25 & 38.2 & .204 \\
\hline 1. & 1.60 & 1.79 & 2.27 & 2.88 & 68.7 & .0911 & .755 & 6.26 & 42.9 & . 1111 \\
\hline 1.5 & 2.31 & 1.57 & 2.39 & 3.63 & 103. & .0607 & .617 & 6.27 & 44.6 & .0667 \\
\hline 2. & 3.12 & 1.32 & 2.34 & 4.13 & 138. & .0455 & .534 & 6.27 & 44.1 & .0523 \\
\hline 2.5 & 3.99 & 1.12 & 2.24 & 4.48 & 172. & .0364 & .478 & 6.27 & 43.2 & .0454 \\
\hline 3. & 4.89 & .966 & 2.14 & 4.73 & 207. & .0304 & .436 & 6.28 & 42.3 & .0417 \\
\hline 3.5 & 5.82 & .846 & 2.04 & 4.92 & 241. & .0260 & .404 & 6.28 & 41.5 & .0393 \\
\hline 4. & 6.76 & .750 & 1.95 & 5.07 & 276 . & .0227 & .377 & 6.28 & 40.8 & .0374 \\
\hline 4.5 & 7.71 & .673 & 1.87. & 5.19 & 310. & .0203 & .357 & 6.28 & 40.2 & .0364 \\
\hline 5. & 8.67 & .610 & 1.80 & 5.29 & 345 . & .0182 & .338 & 6.28 & 39.8 & .0355 \\
\hline
\end{tabular}

$\langle 1.15,35\rangle$

\begin{tabular}{l|l|l|l|l|c|c|c|c|c|c}
\hline 0 & 1 & 0 & 0 & 0 & 0 & $\infty$ & $\infty$ & 6.28 & 0 & $\infty$ \\
.1 & .886 & .521 & .490 & .461 & 7.75 & .786 & 2.19 & 6.09 & 8.75 & 19.9 \\
.3 & .948 & 1.15 & 1.12 & 1.09 & 23.9 & .260 & 1.27 & 6.22 & 25.2 & 1.30 \\
.5 & 1.08 & 1.58 & 1.64 & 1.70 & 40.0 & .156 & .987 & 6.24 & 37.1 & .363 \\
.7 & 1.26 & 1.78 & 1.99 & 2.23 & 56.1 & .112 & .835 & 6.25 & 44.6 & .176 \\
1. & 1.60 & 1.79 & 2.27 & 2.88 & 80.2 & .0781 & .699 & 6.26 & 50.1 & .0949 \\
1.5 & 2.31 & 1.57 & 2.39 & 3.63 & 120. & .0520 & .571 & 6.27 & 52.1 & .0572 \\
2. & 3.12 & 1.32 & 2.34 & 4.13 & 161. & .0390 & .495 & 6.27 & 51.5 & .0449 \\
2.5 & 3.99 & 1.12 & 2.24 & 4.48 & 201. & .0312 & .443 & 6.28 & 50.4 & .0390 \\
3. & 4.89 & .966 & 2.14 & 4.73 & 241. & .0260 & .404 & 6.28 & 49.3 & .0358 \\
3.5 & 5.82 & .846 & 2.04 & 4.92 & 282. & .0223 & .374 & 6.28 & 48.4 & .0337 \\
4. & 6.76 & .750 & 1.95 & 5.07 & 322. & .0195 & .350 & 6.28 & 47.6 & .0322 \\
4.5 & 7.71 & .673 & 1.87 & 5.19 & 362. & .0173 & .330 & 6.28 & 47.0 & .0312 \\
5. & 8.67 & .610 & 1.80 & 5.29 & 402. & .0156 & .313 & 6.28 & 46.4 & .0304 \\
\hline & & & & & $<1.15,40>$ & & & & \\
& & & & & & & & \\
0 & 1 & 0 & 0 & 0 & 0 & $\infty$ & $\infty$ & 6.28 & 0 & $\infty$ \\
.1 & .884 & .508 & .477 & .449 & 8.90 & .686 & 2.05 & 6.11 & 10.1 & 18.4 \\
.3 & .948 & 1.14 & 1.11 & 1.08 & 27.3 & .228 & 1.19 & 6.22 & 28.8 & 1.14 \\
.5 & 1.08 & 1.58 & 1.63 & 1.70 & 45.7 & .137 & .924 & 6.24 & 42.5 & .320 \\
.7 & 1.26 & 1.77 & 1.99 & 2.23 & 64.2 & .0976 & .781 & 6.26 & 51.0 & .154 \\
1. & 1.60 & 1.79 & 2.27 & 2.87 & 91.7 & .0683 & .654 & 6.27 & 57.2 & .0831 \\
1.5 & 2.31 & 1.57 & 2.39 & 3.63 & 138. & .0455 & .534 & 6.27 & 59.6 & .0501 \\
2. & 3.12 & 1.32 & 2.34 & 4.12 & 184. & .0342 & .463 & 6.27 & 58.9 & .0392 \\
2.5 & 3.99 & 1.12 & 2.24 & 4.47 & 230. & .0273 & .414 & 6.28 & 57.6 & .0342 \\
3. & 4.89 & .966 & 2.14 & 4.73 & 276. & .0228 & .378 & 6.28 & 56.4 & .0313 \\
3.5 & 5.82 & .846 & 2.04 & 4.92 & 322. & .0195 & .350 & 6.28 & 55.4 & .0295 \\
4 & 6.75 & .750 & 1.95 & 5.07 & 368. & .0171 & .327 & 6.28 & 54.5 & .0282 \\
4.5 & 7.71 & .673 & 1.87 & 5.19 & 414. & .0152 & .309 & 6.28 & 53.7 & .0273 \\
5 & 8.67 & .610 & 1.80 & 5.29 & 460. & .0137 & .293 & 6.28 & 53.0 & .0266 \\
\hline
\end{tabular}


$\langle 1.20,20\rangle$

\begin{tabular}{|c|c|c|c|c|c|c|c|c|c|c|}
\hline$x$ & $r$ & $a$ & $A_{0}$ & $A$ & $r^{\prime}$ & $a^{\prime}$ & $A_{0}^{\prime}$ & $A^{\prime}$ & $\mathbf{\Omega}$ & Q \\
\hline 0 & 1 & 0 & 0 & 0 & 0 & $\infty$ & $\infty$ & 6.28 & 0 & $\infty$ \\
\hline .1 & .866 & .677 & .630 & .586 & 4.40 & 1.34 & 2.80 & 5.88 & 5.08 & 19.8 \\
\hline .3 & .919 & 1.28 & 1.22 & 1.18 & 14.0 & .437 & 1.64 & 6.14 & 15.3 & 1.79 \\
\hline .5 & 1.05 & 1.70 & 1.74 & 1.79 & 23.6 & .262 & 1.27 & 6.19 & 22.5 & .534 \\
\hline .7 & 1.24 & 1.88 & 2.09 & 2.33 & 33.2 & . 187 & 1.08 & 6.22 & 26.8 & .266 \\
\hline 1. & 1.59 & 1.86 & 2.35 & 2.97 & 47.6 & .131 & .904 & 6.24 & 29.9 & .147 \\
\hline 1.5 & 2.32 & 1.60 & 2.44 & 3.72 & 71.7 & .0873 & .739 & 6.25 & 30.9 & .0916 \\
\hline 2. & 3.14 & 1.34 & 2.38 & 4.20 & 95.7 & .0654 & .640 & 6.26 & 30.5 & .0726 \\
\hline 2.5 & 4.01 & 1.13 & 2.27 & 4.54 & 120 & .0524 & .573 & 6.27 & 29.8 & .0639 \\
\hline 3. & 4.92 & .973 & 2.16 & 4.79 & 144 & .0436 & .523 & 6.27 & 29.2 & .0588 \\
\hline 3.5 & 5.85 & .850 & 2.06 & 4.97 & 168 & .0374 & .484 & 6.27 & 28.7 & .0555 \\
\hline 4. & 6.80 & .753 & 1.96 & 5.12 & 192 & .0327 & .453 & 6.27 & 28.2 & .0534 \\
\hline 4.5 & 7.75 & .675 & 1.88 & 5.23 & 216 & .0291 & .427 & 6.27 & 27.8 & .0517 \\
\hline 5. & 8.72 & .612 & 1.81 & 5.33 & 240 & .0262 & .405 & 6.27 & 27.5 & .0504 \\
\hline \multicolumn{11}{|c|}{$\langle 1.20,30\rangle$} \\
\hline 0 & 1 & 0 & 0 & 0 & 0 & $\infty$ & $\infty$ & 6.28 & 0 & $\infty$ \\
\hline .1 & .854 & .610 & .564 & .521 & 6.81 & .881 & 2.30 & 6.00 & 7.98 & 16.6 \\
\hline .3 & .916 & 1.25 & 1.20 & 1.15 & 21.3 & .291 & 1.34 & 6.19 & 23.2 & 1.25 \\
\hline .5 & 1.05 & 1.69 & 1.73 & 1.77 & 35.6 & .175 & 1.04 & 6.22 & 33.9 & .362 \\
\hline .7 & 1.24 & 1.87 & 2.08 & 2.31 & 50.0 & .125 & .882 & 6.24 & 40.5 & .180 \\
\hline 1. & 1.59 & 1.86 & 2.35 & 2.96 & 71.7 & .0873 & .739 & 6.25 & 45.0 & .0990 \\
\hline 1.5 & 2.32 & 1.60 & 2.44 & 3.71 & 108. & .0582 & .604 & 6.27 & 46.6 & .0613 \\
\hline 2. & 3.13 & 1.34 & 2.37 & 4.20 & 144. & .0436 & .523 & 6.27 & 45.9 & .0487 \\
\hline 2.5 & 4.01 & 1.13 & 2.27 & 4.54 & 180. & .0349 & .468 & 6.27 & 44.8 & .0426 \\
\hline 3. & 4.92 & .973 & 2.16 & 4.78 & 216 & .0291 & .427 & 6.27 & 43.8 & .0392 \\
\hline 3.5 & 5.85 & .850 & 2.06 & 4.97 & 252 & .0249 & .395 & 6.27 & 43.0 & .0370 \\
\hline 4. & 6.79 & .753 & 1.96 & 5.11 & 288. & .0218 & .370 & 6.27 & 42.3 & .0355 \\
\hline 4.5 & 7.75 & .675 & 1.88 & 5.23 & 324 . & .0194 & .349 & 6.28 & 41.8 & .0345 \\
\hline 5. & 8.71 & .611 & 1.80 & 5.33 & 360 . & .0174 & .331 & 6.28 & 41.3 & .0336 \\
\hline \multicolumn{11}{|c|}{$\langle 1.20,40\rangle$} \\
\hline 0 & 1 & 0 & 0 & 0 & 0 & $\infty$ & $\infty$ & 6.28 & 0 & $\infty$ \\
\hline .1 & .849 & .572 & .527 & .485 & 9.22 & .658 & 2.00 & 6.07 & 10.9 & 14.4 \\
\hline .3 & .915 & 1.24 & 1.19 & 1.14 & 28.4 & .218 & 1.16 & 6.21 & 31.1 & .963 \\
\hline .5 & 1.05 & 1.68 & 1.72 & 1.76 & 47.6 & .131 & .904 & 6.24 & 45.4 & .276 \\
\hline .7 & 1.24 & 1.87 & 2.07 & 2.31 & 66.9 & .0935 & .764 & 6.25 & 54.1 & .136 \\
\hline 1. & 1.59 & 1.86 & 2.34 & 2.96 & 95.7 & .0654 & .640 & 6.26 & 60.2 & .0746 \\
\hline 1.5 & 2.31 & 1.60 & 2.44 & 3.71 & 144. & .0436 & .523 & 6.27 & 62.1 & .0461 \\
\hline 2. & 3.13 & 1.34 & 2.37 & 4.20 & 192. & .0327 & .453 & 6.27 & 61.2 & .0365 \\
\hline 2.5 & 4.01 & 1.13 & 2.27 & 4.54 & 240 . & .0262 & .405 & 6.27 & 59.8 & .0320 \\
\hline 3. & 4.92 & .972 & 2.16 & 4.78 & 288. & .0218 & .370 & 6.27 & 58.5 & .0294 \\
\hline 3.5 & 5.85 & .849 & 2.05 & 4.97 & 336. & .0187 & .343 & 6.28 & 57.4 & .0279 \\
\hline 4. & 6.79 & .753 & 1.96 & 5.11 & 384. & .0164 & .320 & 6.28 & 56.5 & .0267 \\
\hline 4.5 & 7.75 & .675 & 1.88 & 5.23 & 432. & .0145 & .302 & 6.28 & 55.7 & .0258 \\
\hline 5. & 8.71 & .611 & 1.80 & 5.33 & 480. & .0131 & .287 & 6.28 & 55.0 & .0252 \\
\hline
\end{tabular}


$\langle 1.50,50\rangle$

\begin{tabular}{|c|c|c|c|c|c|c|c|c|c|c|}
\hline$x$ & r & $a$ & $A_{0}$ & $A$ & $r^{\prime}$ & $a^{\prime}$ & $A_{0}^{\prime}$ & $A^{\prime}$ & $\Omega$ & $Q$ \\
\hline 0 & 1 & 0 & 0 & 0 & 0 & $\infty$ & $\infty$ & 6.28 & 0 & $\infty$ \\
\hline .1 & .682 & .895 & .740 & .611 & 14.3 & .420 & 1.59 & 6.01 & 21.0 & 4.61 \\
\hline .3 & .767 & 1.82 & 1.60 & 1.40 & 44.3 & .140 & .930 & 6.19 & 57.8 & .340 \\
\hline .5 & .928 & 2.29 & 2.20 & 2.12 & 74.3 & .0838 & .722 & 6.22 & 80.0 & .107 \\
\hline .7 & 1.15 & 2.37 & 2.54 & 2.72 & 104. & .0598 & .611 & 6.24 & 90.9 & .0581 \\
\hline 1. & 1.55 & 2.18 & 2.72 & 3.38 & 149. & .0419 & .512 & 6.25 & 96.2 & .0355 \\
\hline 1.5 & 2.34 & 1.75 & 2.68 & 4.10 & 224. & .0279 & .418 & 6.26 & 95.9 & .0244 \\
\hline 2. & 3.21 & 1.42 & 2.53 & 4.54 & 299. & .0210 & .362 & 6.27 & 93.3 & .0204 \\
\hline 2.5 & 4.12 & 1.17 & 2.38 & 4.84 & 374. & .0168 & .324 & 6.27 & 90.9 & .0185 \\
\hline 3. & 5.06 & .998 & 2.24 & 5.05 & 449. & .0140 & 296 & 6.27 & 88.9 & .0174 \\
\hline 3.5 & 6.01 & .866 & 2.12 & 5.21 & 524. & .0120 & .274 & 6.28 & 87.3 & .0166 \\
\hline 4. & 6.97 & .764 & 2.02 & 5.33 & 599. & .0105 & .256 & 6.28 & 86.0 & .0161 \\
\hline 4.5 & 7.94 & .683 & 1.93 & 5.42 & 674. & .00931 & .242 & 6.28 & 84.9 & .0158 \\
\hline 5. & 8.92 & .617 & 1.84 & 5.50 & 749. & .00838 & .229 & 6.28 & 84.0 & .0155 \\
\hline
\end{tabular}




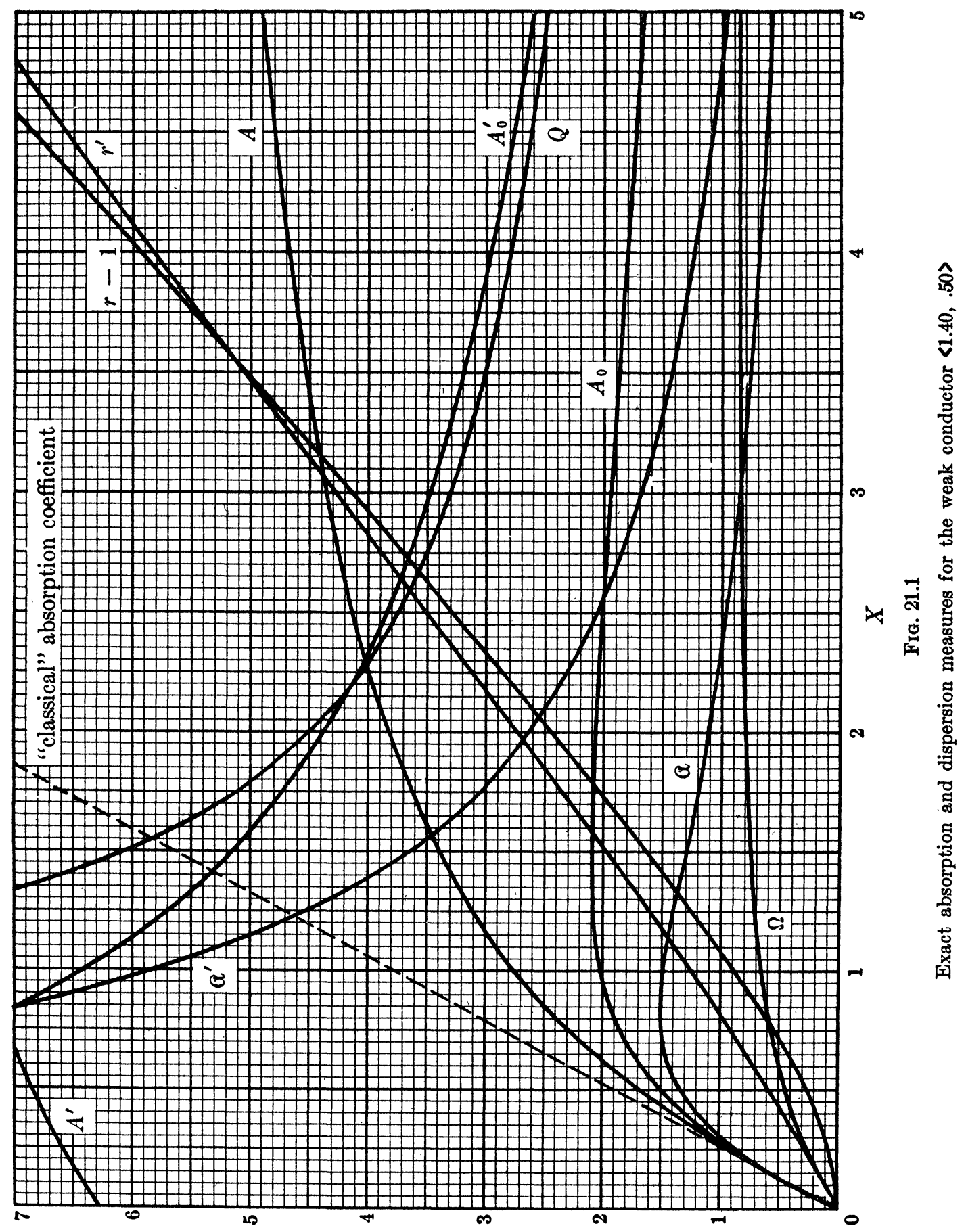




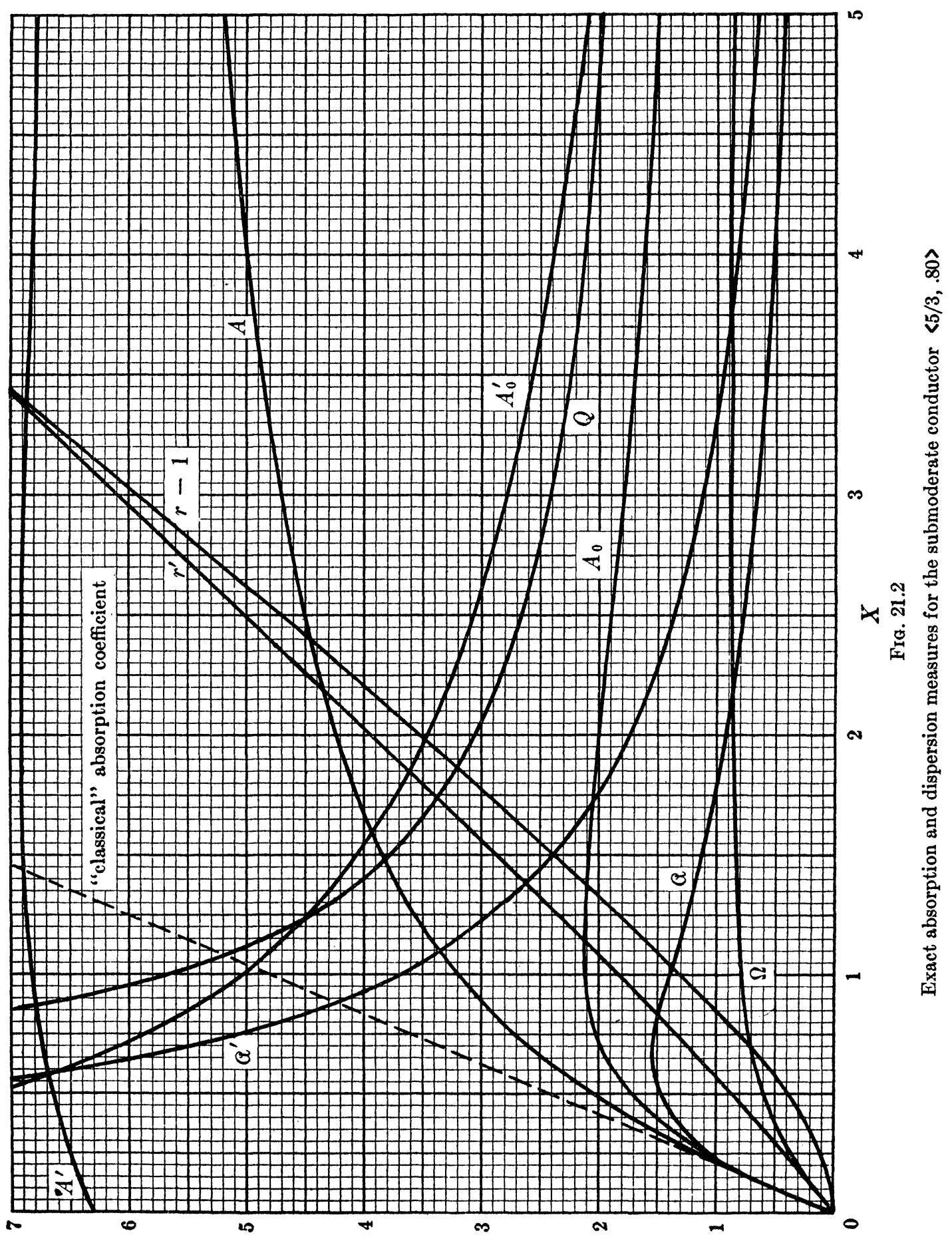




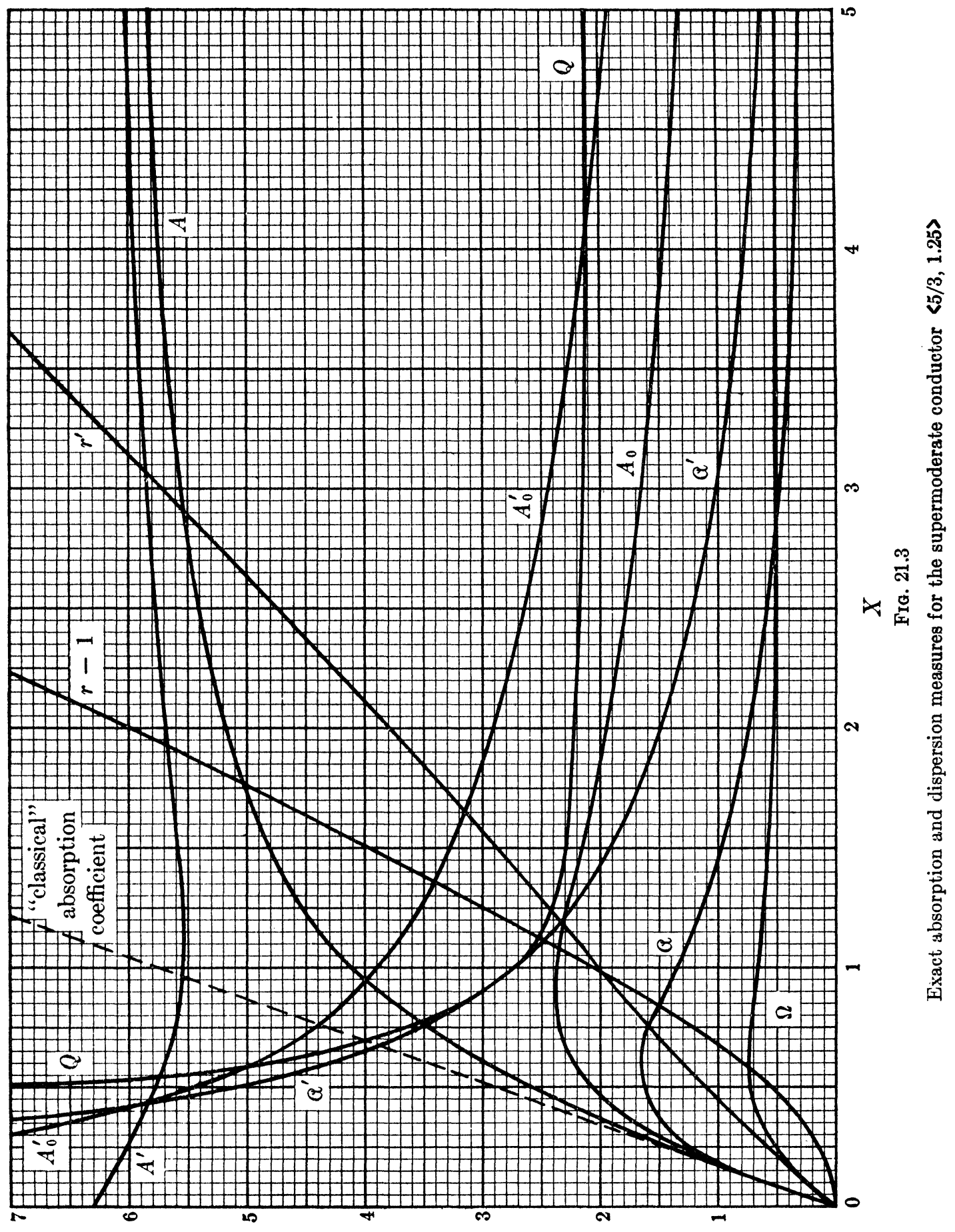


C. TRUESDELL

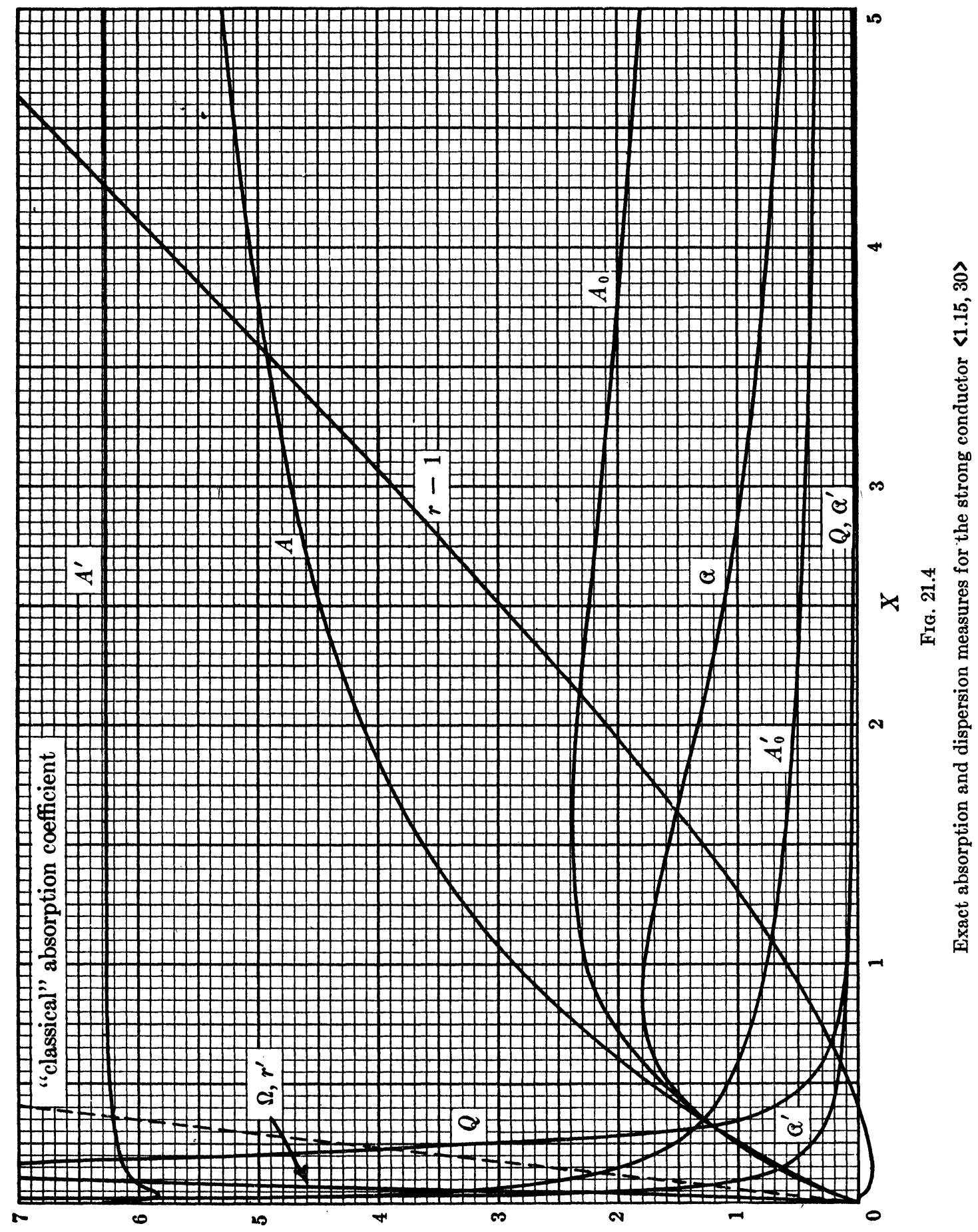




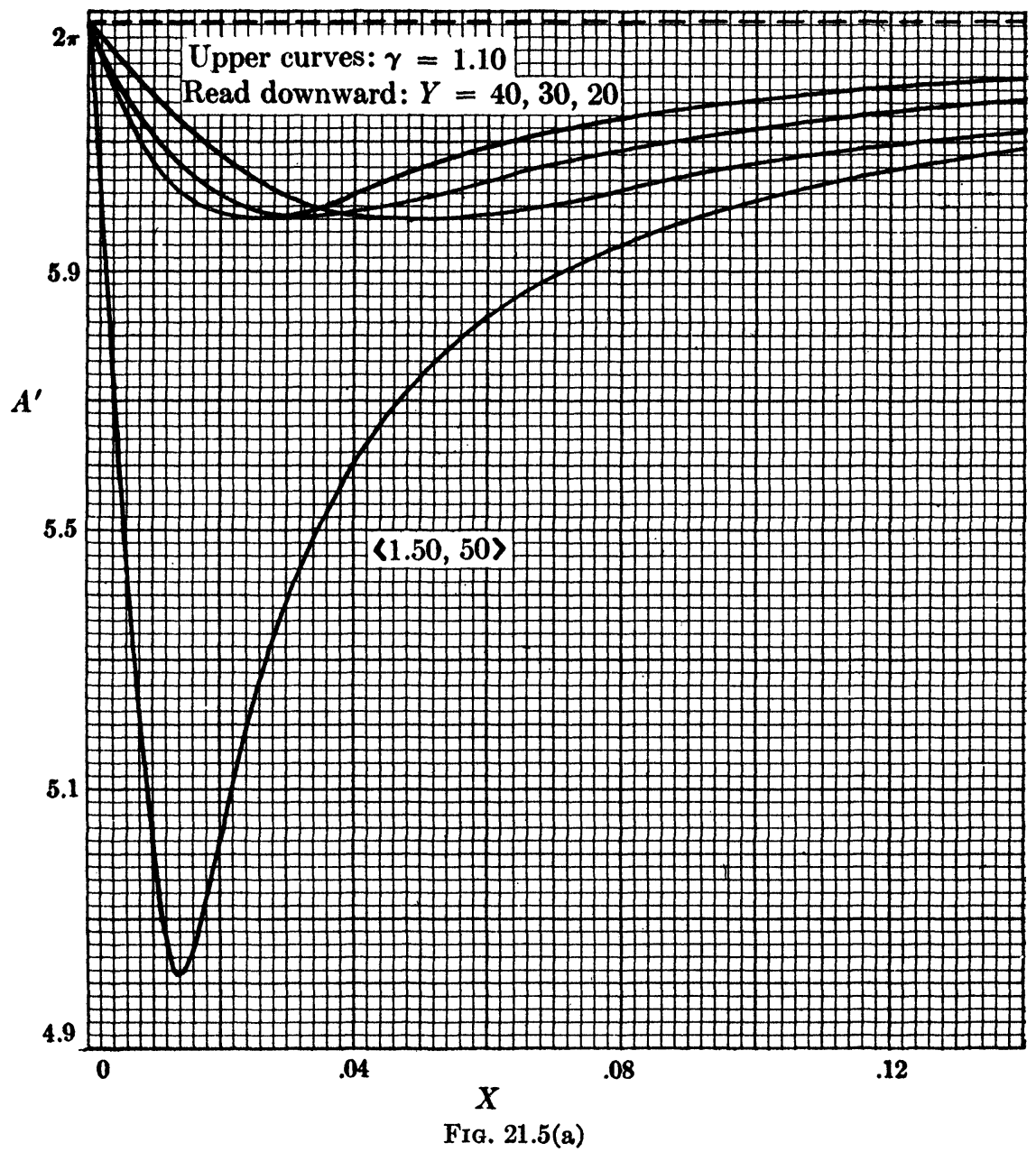

The minimum in $A^{\prime}$ for certain strong conductors 


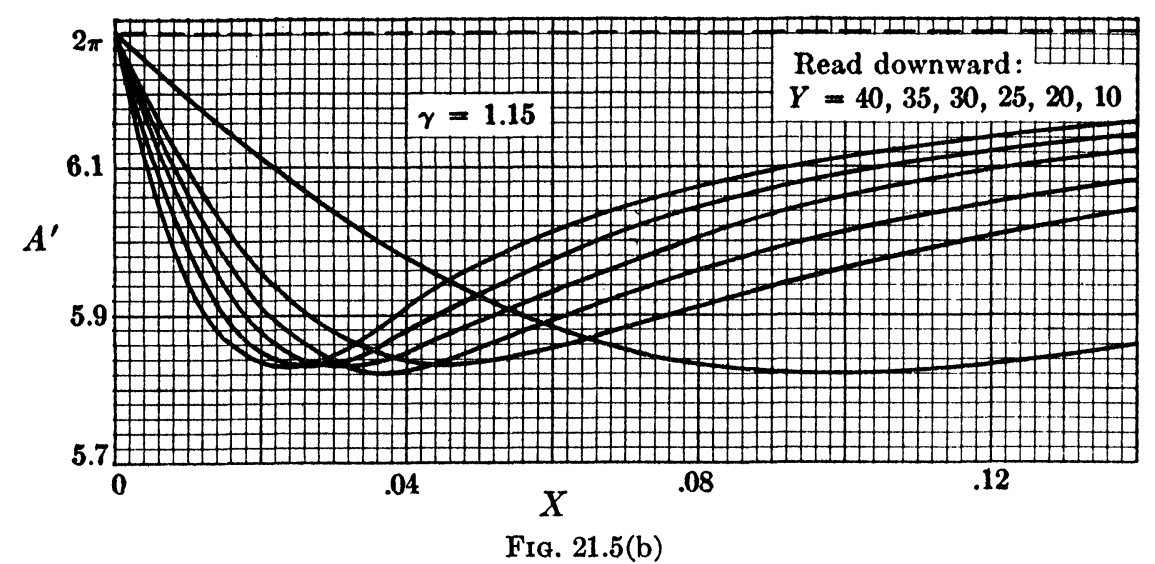

The minimum in $A^{\prime}$ for certain strong conductors

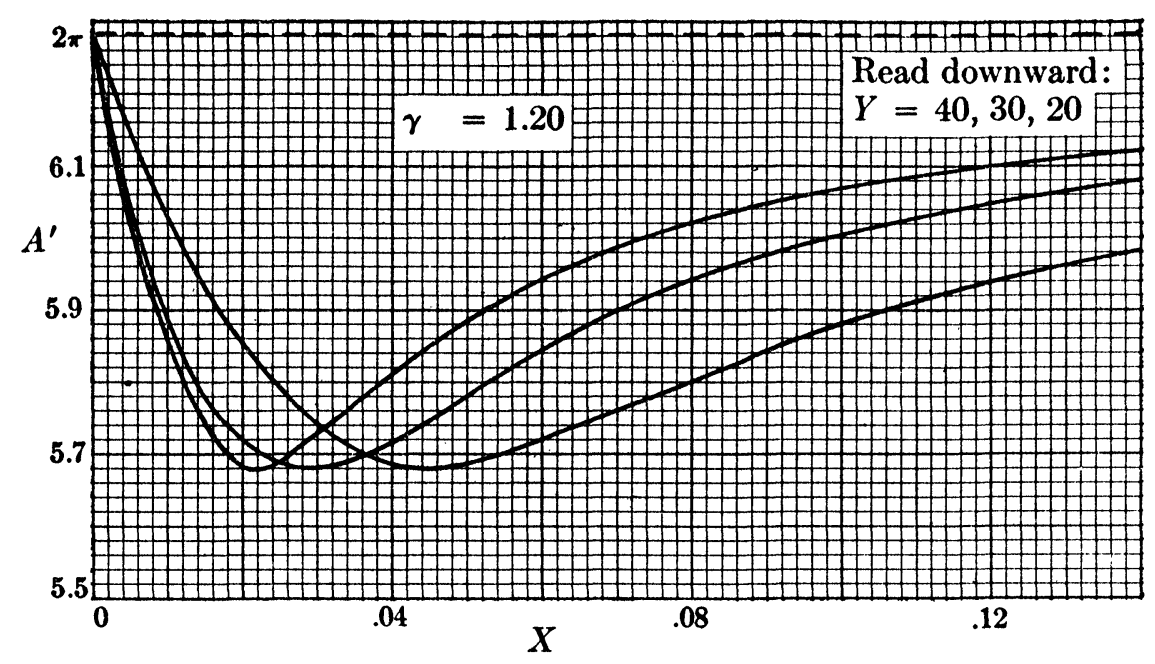

Fig. 21.5(c)

The minimum in $A^{\prime}$ for certain strong conductors 


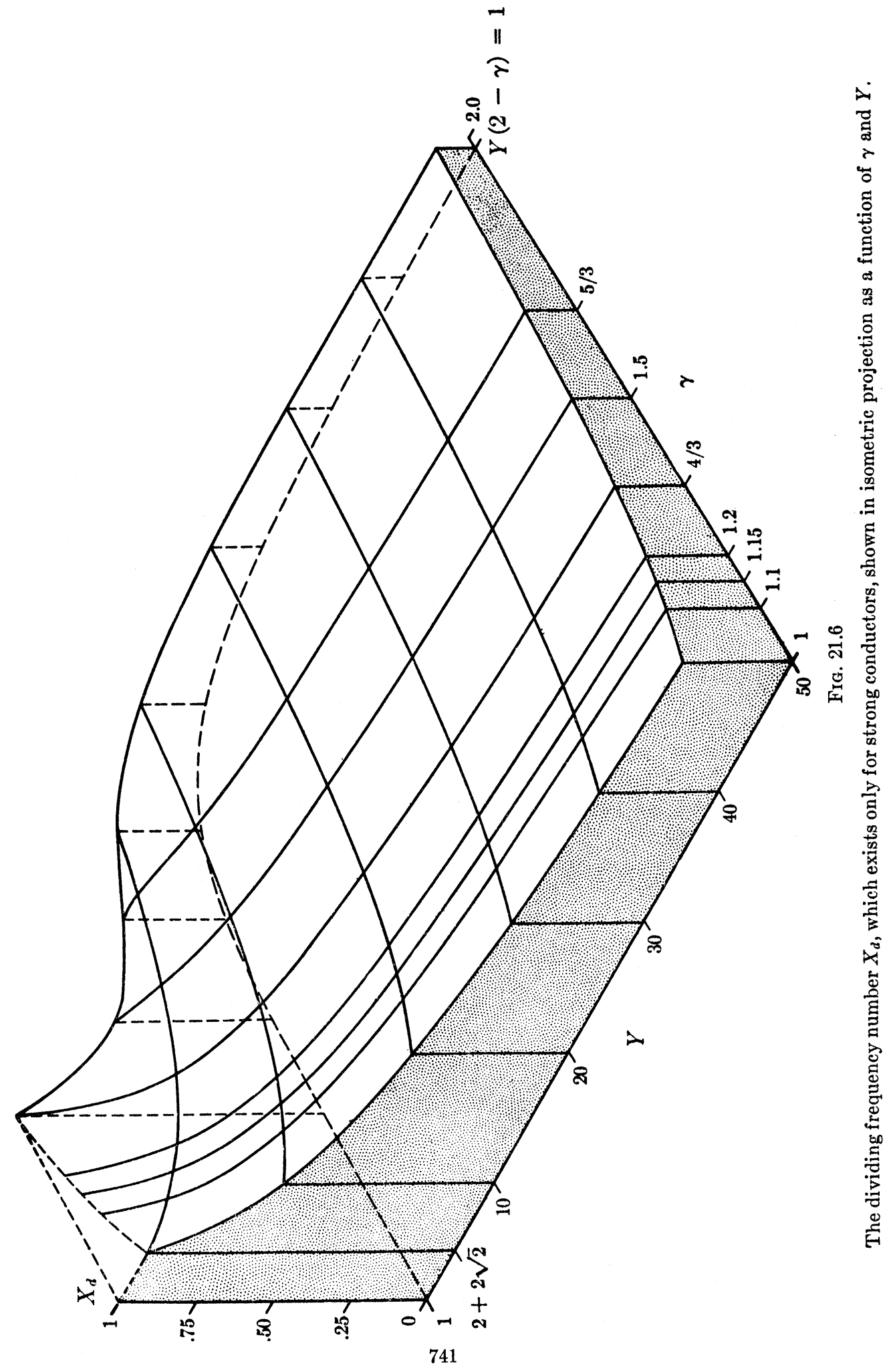

